\author{
Federal Reserve Bank of New York \\ Staff Reports
}

\title{
Time-Varying Inflation Risk and Stock Returns
}

\author{
Martijn Boons \\ Fernando M. Duarte \\ Frans de Roon \\ Marta Szymanowska
}

Staff Report No. 621

May 2013

Revised April 2019

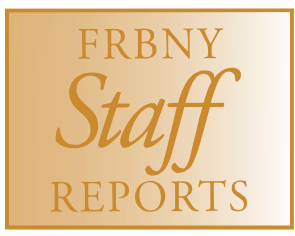

This paper presents preliminary findings and is being distributed to economists and other interested readers solely to stimulate discussion and elicit comments. The views expressed in this paper are those of the authors and do not necessarily reflect the position of the Federal Reserve Bank of New York or the Federal Reserve System. Any errors or omissions are the responsibility of the authors. 
Time-Varying Inflation Risk and Stock Returns

Martijn Boons, Fernando M. Duarte, Frans de Roon, and Marta Szymanowska

Federal Reserve Bank of New York Staff Reports, no. 621

May 2013; revised April 2019

JEL classification: G11, G12, G13

\begin{abstract}
We show that inflation risk is priced in stock returns and that inflation risk premia in the crosssection and the aggregate market vary over time, even changing sign as in the early 2000s. This time variation is due to both price and quantities of inflation risk changing over time. Using a consumption-based asset pricing model, we argue that inflation risk is priced because inflation predicts real consumption growth. The historical changes in this predictability and in stocks' inflation betas can account for the size, variability, predictability and sign reversals in inflation risk premia.
\end{abstract}

Key words: inflation, time-varying inflation risk premium, inflation hedging, individual stock returns, cross-sectional asset pricing, nominal-real covariance

Duarte: Federal Reserve Bank of New York. Boons (corresponding author): Nova School of Business and Economics (email: martijn.boons@novasbe.pt). De Roon: Tilburg School of Economics and Management. Szymanowska: Rotterdam School of Management, Erasmus University. The authors thank an anonymous referee, Tobias Adrian, Hengjie Ai, Christine Breiner, Ricardo Caballero, Hui Chen, Richard Crump, Frank de Jong, Joost Driessen, Maya Eden, Rik Frehen, Xavier Gabaix, Jonathan Goldberg, Leonid Kogan, Jennifer La'O, Guido Lorenzoni, Gustavo Manso, Matt Notowididgo, Sahar Parsa, Pedro Santa-Clara, Piet Sercu, Alp Simsek, Ken Singleton, Dongho Song, Laura Spierdijk, Adrien Verdelhan, Luis Viceira and Ivo Welch; conference participants at the EFA annual meeting 2018, Auckland Finance Meeting 2013, Dauphine-Amundi Annual Workshop 2013, ESSFM Gerzensee 2012, LUBRAFIN 2014, and Netspar International Pension Workshop 2012; and seminar participants at Rotterdam School of Management, Tilburg University, University of Auckland, MIT, MIT Sloan School of Management, Duke University's Fuqua School of Business, Tepper School of Business, Smith School of Business, Federal Reserve Board, and the Federal Reserve Bank of New York. This paper has received financial support from the Dauphine Chair in Asset Management, an initiative of Amundi and the University Paris-Dauphine, under the aegis of the Dauphine Foundation.This paper replaces and supersedes two papers formerly circulated as "The Stock Market Price of Inflation Risk and Its Variation over Time," by Martijn Boons, Frans de Roon, and Marta Szymanowska, and "Inflation Risk and the Cross Section of Stock Returns," by Fernando Duarte. This paper was previously issued under the title "Time-Varying Inflation Risk and the Cross Section of Stock Returns." The views expressed in this paper are those of the authors and do not necessarily reflect the position of the Federal Reserve Bank of New York or the Federal Reserve System. 


\section{Introduction}

We show that inflation risk is priced in stock returns and that both the price and quantities of inflation risk are strongly time-varying, even changing sign over our sample from 1962 to 2014. We argue that inflation is risky because it predicts real consumption growth in a time-varying way. Positive shocks to inflation sometimes contain bad news for future consumption, whereas at other times they contain good news. We provide new empirical evidence, for both the cross-section of stock returns and the aggregate stock market, and develop an equilibrium model to argue that it is the time-variation in the predictive content of inflation that drives the observed time-variation in the price and quantities of inflation risk.

To quantify the predictive content of inflation at a given point in time, we define the nominal-real covariance as the slope coefficient in a conditional (that is, rolling) regression of real consumption growth on lagged inflation. We find that this nominal-real covariance is an economically strong and statistically significant predictor of the inflation risk premium in the cross-section of stocks. We measure the quantity of inflation risk of stocks by their conditional inflation beta, that is, the slope coefficient in a rolling regression of returns on inflation shocks. A one standard deviation increase in the nominal-real covariance predicts an increase in annualized expected return of $5.3 \%$ for a high-minus-low portfolio constructed by sorting stocks in deciles according to their inflation beta. Given an unconditional average return for the high-minus-low inflation beta portfolio of $-4.2 \%$, this increase implies that the inflation risk premium can switch sign.

To understand why, consider periods when the nominal-real covariance is negative, such as during the 1970s. In such periods, higher inflation is a bad state of nature as it predicts lower future consumption growth. As a result, high inflation beta stocks are attractive to hedge against bad states and therefore command lower premia. In contrast, in periods when the nominal-real covariance is positive, such as since the early 2000s, a positive shock to inflation contains good news for future consumption. Now, stocks with lower inflation betas are more attractive to hedge and the inflation risk premium switches sign.

We provide further evidence for this explanation by running pooled predictive regressions for the returns of ten inflation beta-sorted portfolios on their conditional inflation betas, the nominal-real covariance, and an interaction term. We find that the interaction term predicts returns well, whereas the other two (noninteraction) terms do not. Thus, understanding the cross-section of inflation risk premia requires knowledge not only of the relative magnitude of inflation betas across stocks, but also of the nominal-real covariance at each point in time.

To understand how variation in quantities and price of inflation risk interact to determine inflation risk premia, we decompose conditional inflation betas into portfolio-specific and time-specific components in our pooled regressions. We present three results that are new to the literature. First, the cross-sectional distribution of inflation risk premia widens and compresses over time due to variation in the price of inflation risk. This result follows from the fact that the interaction between the portfolio-specific component of inflation 
betas and the nominal-real covariance fully captures the time-series predictability of the high-minus-low inflation beta portfolio return. Time-variation in the relative quantities of inflation risk (inflation betas) in the cross-section does not add any information.

Second, quantities of inflation risk vary over time with the nominal-real covariance. This time-variation is largely common across stocks. Pairwise correlations between the inflation betas of any two decile portfolios and between any decile portfolio and the aggregate stock market are well above $90 \%$.

Third, the aggregate stock market risk premium contains an inflation component that is time-varying, due to time-variation in both the price and quantity of inflation risk in the aggregate market. This conclusion follows from the fact that the interaction between the inflation beta of the market portfolio and the nominalreal covariance predicts market returns, whereas neither the nominal-real covariance nor the market's inflation beta predict returns in isolation. The joint effect of the price and quantity of inflation risk is large: when the inflation beta of the market portfolio and the nominal-real covariance are both one standard deviation below their mean, the market risk premium equals $13.6 \%$, more than double its unconditional mean. The intuition is that the aggregate stock market is riskier when it comoves negatively with inflation shocks that contain bad news for future consumption growth.

To provide a theoretical foundation for this empirical evidence, we develop a consumption-based equilibrium model. Our model takes the joint stochastic processes for consumption, inflation, and the nominal-real covariance as given, while asset prices are determined endogenously through the Euler equation of a representative agent. There are three key ingredients in our model. The first ingredient is that the representative agent has Epstein-Zin-Weil (EZ) utility. The second ingredient is that inflation is persistent. ${ }^{1}$ The third ingredient is that, as in the data, inflation shocks predict future real consumption growth in a time-varying way that allows for sign reversals. ${ }^{2}$ It is this third ingredient that is new to the literature.

Because EZ preferences are not time-separable, marginal utility depends not only on current consumption growth, as in the classic consumption-CAPM, but also on the entire future path of consumption. Since inflation shocks predict future consumption growth, inflation shocks represent shocks to marginal utility and are therefore priced. When the nominal-real covariance is positive, the price of inflation risk is also positive, because higher inflation today represents a good state of nature as future consumption growth is expected to be higher. As the nominal-real covariance changes sign over time, so does the price of inflation risk. To study the cross-section of stock returns, we model dividends as being exogenous and exposed to the same sources of risk as consumption. Because inflation shocks predict the future path of dividends with sign and magnitude depending on the nominal-real covariance, the endogenous quantities of inflation risk

\footnotetext{
${ }^{1}$ Inflation persistence is widely documented in the literature (see, e.g., Fuhrer and Moore, 1995; Stock and Watson, 2005; Campbell and Viceira, 2002; Ang, Bekaert, and Wei, 2007).

${ }^{2}$ Although interesting, our aim is not to explain the source of this predictability. Rather, our aim is to explain the asset pricing implications from exogenous variation in the predictive relation between inflation and consumption growth. See Duffee (2018) for a survey and the challenges in explaining the time-variation in the nominal-real covariance.
} 
of stocks are also functions of the nominal-real covariance. Our proposed calibration shows that the model can quantitatively reproduce the empirical results discussed above, including the size and time-variation of the price and quantities of inflation risk in the cross-section of stocks, and their relation to the nominal-real covariance. Inflation persistence is key in allowing us to quantitatively match the relatively high inflation risk premium observed in the data, as a more persistent inflation has a longer-lasting effect on the future path of expected consumption and hence on marginal utility.

Empirically, we find that the cross-section of inflation betas is wide, which is instrumental in identifying the inflation risk premium. Our evidence suggests that the cross-sectional variation in inflation betas is mainly driven by variation in inflation exposure in cash flows rather than in discount rates. There is heterogeneity in inflation betas both across and within industries. Industry affiliation is an important determinant of inflation risk unconditionally. On average, the best inflation hedgers are oil, gold, and mining stocks. However, inflation risk is also priced in a time-varying way within industries, even outside the best inflation-hedging industries. This finding is new to the literature and motivates us to abstract from industry effects in our model.

Our empirical results are robust. We consider alternative measures of the nominal-real covariance, using either the covariance between inflation and industrial production or the stock market beta of a long-term bond. We analyze alternative ways to measure inflation risk premia, estimating a maximum correlation mimicking portfolio (Breeden, Gibbons, and Litzenberger, 1989) and a Fama and MacBeth (1973) crosssectional regression for individual stocks. We use different methods to estimate inflation risk, for instance, by filtering inflation shocks using first-differences or an AR(1)-process instead of an ARMA(1,1)-process, which is our main specification. We also consider a truly out-of-sample exercise using real-time vintage inflation, similar to Ang, Brière, and Signori (2012). We find that our inflation beta-sorted portfolios are not only exposed to shocks in realized inflation but also to changes in breakeven inflation from TIPS. Finally, our results carry through when we control for benchmark predictors or exposure to benchmark asset pricing factors. $^{3}$

We contribute to the empirical literature on inflation by providing a rich set of new facts concerning inflation risk premia in the stock market. Measures of the inflation risk premium have had a natural starting point in the yield curve. With the development of sophisticated no-arbitrage term structure models and the emergence of inflation-linked bonds, estimates of the inflation risk premium in the bond market have become more reliable and widely available. ${ }^{4}$ Another conventional way to estimate the inflation risk premium is to

\footnotetext{
${ }^{3}$ The benchmark predictors are the dividend yield, term spread, default spread, and the consumption-wealth ratio of Lettau and Ludvigson (2001a,b). The benchmark factors are MKT, SMB, HML, WML, RMW, and CMA combined in the CAPM (Sharpe, 1964; Lintner, 1965; Mossin, 1966) and the multi-factor models of Fama and French (1993), Carhart (1997), and Fama and French (2015).

${ }^{4}$ See, e.g., Ang and Piazzesi (2003); Ang, Piazzesi, and Wei (2006); Ang, Bekaert, and Wei (2007, 2008); Dai, Singleton, and Yang (2007); Le, Singleton, and Dai (2010); Le and Singleton (2010); Haubrich, Pennacchi, and Ritchken (2012); Gürkaynak, Sack, and Wright (2010); Chen, Liu, and Cheng (2010); Campbell, Sunderam, and Viceira (2017).
} 
study the joint time-series behavior of inflation and aggregate market returns. ${ }^{5}$

Similar to us, Chen, Roll, and Ross (1986), Ferson and Harvey (1991), and Ang, Brière, and Signori (2012) estimate the inflation risk premium in the cross-section of stocks. However, these papers focus on the unconditional asset pricing implications of inflation risk. The typical point estimate is a negative inflation risk premium that is not statistically significant and varies considerably across sample periods. We provide a natural explanation for this evidence, which is a time-varying inflation risk premium that can change sign. We also contribute to this literature by studying the macroeconomic mechanism behind this time-variation, both empirically and in an equilibrium model.

Our model builds on the mathematics and economic intuition of the long-run risk model of Bansal and Yaron (2004), but the economic sources of risk are different. We contribute to the long-run risk literature in several ways. First, we use directly observable inflation as a predictor of consumption instead of an unobserved long-run risk component that must be inferred from asset prices using the model's assumptions. The observability of inflation thus resolves one of the main limitations of the long-run risk model. Our reliance on observables is advantageous also relative to other consumption-based models that contain varying degrees of "dark matter" (Chen, Dou, and Kogan, 2015; Cochrane, 2017). Second, predictability of consumption using inflation, and inflation persistence itself, both operate at business cycle frequency. In contrast, longrun risk operates at substantially lower frequencies. In addition, inflation shocks have a higher variance than long-run risk shocks. The combination of higher volatility and lower persistence of inflation shocks makes the inflation premium comparable in magnitude to the premium earned by the low volatility, but high persistence, long-run risk shocks. Third, because inflation is less persistent than long-run risk, the timing premium that agents are willing to pay for the early resolution of uncertainty in our model is considerably lower than in the long-run risk model by about 50\%, which alleviates the criticism of Epstein, Farhi, and Strzalecki (2014). Finally, inflation predicts consumption growth in a time-varying way as determined by the nominal-real covariance, while the predictability of consumption with long-run risk is constant over time.

Recent articles in the bond market literature argue that the nominal-real covariance is an important driver of the time-variation in bond prices and in the comovement of stocks and bonds. Campbell, Sunderam, and Viceira (2017) document a simultaneous change in the sign of the term premium for US Treasuries and the nominal-real covariance at the turn of the century. Campbell, Pflueger, and Viceira (2015) and Song (2017) interpret the time-variation in bond risk through changes in monetary policy. We argue that monetary policy is unlikely to be the main driver of our results. The high and low inflation portfolio are not differentially exposed to monetary policy shocks. Moreover, the nominal-real covariance predicts returns also when controlling for different monetary policy regimes. David and Veronesi (2013) contend that it is the time-varying signaling role of inflation (plus learning) that drives the joint dynamics of stock and bond

\footnotetext{
${ }^{5}$ See, e.g., Modigliani and Cohn (1979); Wachter (2006); Gabaix (2008); Bansal and Shaliastovich (2012).
} 
markets. Kang and Pflueger (2015) find that the nominal-real covariance affects corporate bond yield spreads through a credit channel. The nominal-real covariance also features prominently in recent term structure literature that analyzes the implications of the zero lower bound (see, e.g., Gourio and Ngo, 2016; Bilal, 2017; Bretscher, Hsu, and Tamoni, 2017).

Although we estimate an unconditional inflation risk premium in the bond market that is close to what we estimate in the stock market, our evidence suggests that the nominal-real covariance is not a strong time-series predictor of excess bond returns, consistent with Campbell, Sunderam, and Viceira (2017). ${ }^{6}$ Furthermore, previous papers study the nominal-real covariance as measured by the correlation between the returns of long-term government bonds and the aggregate stock market. To the best of our knowledge, we are the first to show that inflation predicts real consumption growth in a time-varying way, and to use this conditional (and exclusively macroeconomic) relation to measure the nominal-real covariance. We also study the pricing implications of time-variation in the nominal-real covariance in an asset class not studied before (the cross-section of stocks) and present new evidence on the relative importance of price and quantities of risk.

Finally, Gorodnichenko and Weber (2016) show that the conditional volatility of stock returns increases more for firms with stickier prices after monetary policy shocks. Weber (2015) finds that firms with stickier prices are more exposed to monetary policy shocks and earn a premium that is fully explained by market beta, because sticky price firms have more cyclical cash flows and both higher discount rate and cash flow beta. Although we also find that exposure in cash flows is important, and despite inflation risk and price stickiness being related, we find that neither exposure to the market nor to monetary policy shocks explains the variation in inflation premia that we document. Together, these differences suggest that price stickiness and our inflation-sorted portfolios are subject to different sources of risk.

\section{Data}

We use monthly inflation $\left(\Pi_{t}\right)$ calculated as the percentage change in the seasonally-adjusted Consumer Price Index for All Urban Consumers (CPI) available from the U.S. Bureau of Labor Statistics. Monthly per capita real consumption growth $\left(\Delta C_{t}\right)$ is calculated using seasonally-adjusted aggregate nominal consumption expenditures on nondurables and services (from National Income and Product Accounts (NIPA) Table 2.8.5) combined with population numbers (from NIPA Table 2.6) and price deflator series (from NIPA Table 2.8.4). ${ }^{7}$ In our asset pricing tests, we use all ordinary common stocks traded on the NYSE, AMEX, and NASDAQ from CRSP (excluding firms with negative book equity from COMPUSTAT). The CRSP value-weighted market portfolio, the one-month T-bill return, benchmark asset pricing factors, and industry portfolios are

\footnotetext{
${ }^{6}$ Even though in their model, just like in ours, the nominal-real covariance is a key state variable driving risk premia, Campbell, Sunderam, and Viceira (2017) conclude in Section 5.3 that "bond returns have very limited predictability."

${ }^{7}$ In Section 8., we replicate our main result using quarterly consumption data.
} 
from Kenneth French's website. Table 1 presents descriptive statistics for the sample period we use in our tests: July 1962 to December 2014. The start of the sample period coincides with the introduction of AMEX stocks in the CRSP file and is common to many empirical studies of the cross-section.

\section{The Time-Varying Relation Between Inflation and Consumption}

To analyze the conditional relation between current inflation and future real consumption growth, we present in Panel A of Table 2 results from simple OLS regressions of future consumption growth from month $t+1$ to $t+K$ on inflation over month $t$ :

$$
\Delta C_{t+1: t+K}=d_{0}^{K}+d_{1}^{K} \Pi_{t}+e_{t+1: t+K}, K=1,3,6,12 .
$$

We see that the unconditional relation between current inflation and future consumption is negative, as measured by the coefficient estimate for $d_{1}^{K} .8$ Thus, an increase in inflation is bad news historically, which is consistent with evidence in, for instance, Piazzesi and Schneider (2006). However, over our relatively long sample period, the coefficient on inflation is not significantly different from zero for any horizon $K$. A potential explanation for this seeming inconsistency with the existing literature is that the unconditional regression masks variation over time in the relation between consumption and inflation.

To quantify the magnitude of this time-variation, we run the following two-stage test:

$$
\begin{aligned}
\Delta C_{s+1: s+K} & =a_{t}^{K}+b_{t}^{K} \Pi_{s}+e_{s+1: s+K}, \text { for } s=1, \ldots, t-K, \\
\Delta C_{t+1: t+K} & =d_{0}^{c, K}+d_{1}^{c, K}\left(\widehat{a_{t}^{K}}+\widehat{b_{t}^{K}} \Pi_{t}\right)+e_{t+1: t+K} .
\end{aligned}
$$

In the first stage, Equation (2) regresses consumption growth on lagged inflation over a backward-looking window using all data available up to month $t$. Hence, the expanding window $s$ runs from 1 to $t-K$. This regression is estimated using weighted least squares (WLS) where weights are given by an exponential weighting scheme that has a half-life converging to 60 months for large $t$. The expanding window ensures that we use as much information as possible, whereas an exponential decay in weights ascertains that the largest weight is given to the most recent observations. In the second stage, Eq. (3) uses the estimated coefficients and inflation observed at time $t$, that is, $\widehat{a_{t}^{K}}+\widehat{b_{t}^{K}} \Pi_{t}$, to predict consumption growth from $t+1$ to $t+K$. This setup ensures that we use no forward-looking information when we predict consumption growth in the second stage. The absence of a look-ahead bias is important, because we will later use the estimated first-stage coefficient $b_{t}^{K}$ to predict various returns in the time-series. Hence, we prefer our simple setup over more highly parametrized models that require the full sample for estimation, such as a state-space model with time-varying parameters.

\footnotetext{
${ }^{8}$ In the data, we lag inflation by an additional month, to account for the reporting delay of CPI numbers.
} 
If our two-stage structure correctly models the conditional expectation of consumption growth, we should find that $d_{0}^{c, K}=0$ and $d_{1}^{c, K}=1$. Panel B of Table 2 presents the results. To test significance, we report asymptotic Newey-West (with $K$ lags) and bootstrapped $t$-statistics. ${ }^{9}$ The estimate of $d_{1}^{c, K}$ is significantly larger than zero at all horizons. For no horizon $K$ can we reject the hypothesis that $d_{0}^{c, K}=0$ nor that $d_{1}^{c, K}=1$, suggesting that the two-stage structure provides an unbiased estimate of the conditional expectation of consumption growth. Moreover, in the second stage, we capture an economically large share of the variation in consumption growth, with an $R^{2}$ that increases from $3 \%$ at the one month horizon to $15 \%$ at the one year horizon.

Following the literature on predictive regressions (see, e.g., Welch and Goyal, 2007), we also calculate an out-of-sample $R^{2}\left(R^{2}-O O S\right)$. The $R^{2}-O O S$ measures the improvement from conditioning on inflation relative to a conditional model that includes only a time-varying constant, $a_{t}^{K}$, which measures lagged average consumption growth. We find that the $R^{2}-O O S$ is similarly increasing from $2 \%$ at the one month horizon to $10 \%$ at the one year horizon. We conclude that inflation strongly predicts consumption growth when we account for the time-varying relation between these macro-variables.

Our main proxy for the nominal-real covariance, which we henceforth denote $N R C_{t}^{C}$, is the time-varying coefficient from the first stage regression in Eq. (2) of twelve-month consumption growth on lagged inflation, $\widehat{b_{t}^{12}}$. In robustness checks, we consider two alternative proxies for the nominal-real covariance. First, the time-varying relation between inflation and industrial production growth, denoted $N R C_{t}^{I P}$, is estimated by substituting industrial production for consumption in the left-hand side of Eq. (2). ${ }^{10}$ This alternative measure effectively treats inflation as a recession state variable of the type advocated in Cochrane (2005) and Koijen, Lustig, and Van Nieuwerburgh (2017). Second, we consider the measure based on asset prices that is used in Campbell, Pflueger, and Viceira (2015): the negative of the stock market beta of a long-term treasury bond, denoted $N R C_{t}^{-B B}$. We estimate this time-varying beta using a 60 -month rolling window regression of the 10-year constant maturity treasury bond return on the aggregate stock market return.

Fig. 1 plots the three proxies of the nominal-real covariance and shows strong comovement, with correlations around 0.75. Consistent with Campbell, Pflueger, and Viceira (2015), $N R C_{t}^{-B B}$ has changed sign from negative to positive in the early 2000s. In fact, all three measures have increased markedly since the turn of the century, as noted also in Bekaert and Wang (2010), Campbell, Sunderam, and Viceira (2017), and David and Veronesi (2013). In the remainder of this paper, we analyze empirically and theoretically the asset pricing implications of the time-variation in inflation risk driven by this nominal-real covariance.

\footnotetext{
${ }^{9}$ As detailed in Section 1 of the Online Appendix, these standard errors are derived from 1000 block-bootstrap replications of the coefficient estimates to address concerns about errors-in-variables (EIV) bias.

${ }^{10}$ Seasonally-adjusted industrial production growth is from the $F R E D^{\circledR}$ database of the St. Louis FED.
} 


\section{The Time-Varying Inflation Risk Premium}

This section analyzes whether the inflation risk premium varies over time in a manner consistent with the time-variation in the nominal-real covariance.

\subsection{Estimating the Inflation Risk Premium}

We first describe our method to estimate the inflation risk premium.

\subsubsection{Inflation Betas}

At the end of each sample month $t$, we measure the inflation exposure of firm $i$ by estimating its historical "beta" of excess returns, $R_{i, t}$, with respect to monthly innovations in inflation. Following Fama and Gibbons (1984), Vassalou (2000) and Campbell and Viceira (2002), we filter out these innovations, denoted $u_{\Pi, t}$, using an ARMA(1,1)-model. This model empirically captures many of the important dynamics of inflation. ${ }^{11}$

We estimate historical inflation betas in the same way that we estimate the nominal-real covariance in Eq. (2): using a WLS regression with exponential weights over an expanding window that uses all observations from the first month the stock is included in the sample up to month $t$. We require that stocks have at least 24 out of the last 60 months of returns available. The estimator of a stock's inflation exposure, $\beta_{\Pi, i, t}$, is then given by

$$
\begin{aligned}
\left(\widehat{\alpha_{i, t}}, \widehat{\beta_{\Pi, i, t}}\right) & =\underset{\alpha_{i, t}, \beta_{\Pi, i, t}}{\arg \min } \sum_{\tau=1}^{t} K(\tau)\left(R_{i, \tau}-\alpha_{i, t}-\beta_{\Pi, i, t} u_{\Pi, \tau}\right)^{2} \\
\text { with weights } K(\tau) & =\frac{\exp (-|t-\tau| h)}{\sum_{\tau=1}^{t-1} \exp (-|t-\tau| h)} .
\end{aligned}
$$

Using $h=\log (2) / 60$, the half-life of the weights $K(\tau)$ converges to 60 months for large $t$, which is consistent with the standard five-year rolling window used in empirical asset pricing tests. Following Elton, Gruber, and Urich (1978), Cosemans, Frehen, Schotman, and Bauer (2016), and Levi and Welch (2017), we transform the estimated $\widehat{\beta_{\Pi, i, t}}$ using a Vasicek (1973) adjustment

$$
\widehat{\beta_{\Pi, i, t}^{v}}=\widehat{\beta_{\Pi, i, t}}+\frac{\operatorname{var}_{T S}\left(\widehat{\beta_{\Pi, i, t}}\right)}{\left[\operatorname{var}_{T S}\left(\widehat{\beta_{\Pi, i, t}}\right)+\operatorname{var}_{C S}\left(\widehat{\beta_{\Pi, i, t}}\right)\right]}\left[\operatorname{mean}_{C S}\left(\widehat{\beta_{\Pi, i, t}}\right)-\widehat{\beta_{\Pi, i, t}}\right] .
$$

Thus, each $\widehat{\beta_{\Pi, i, t}^{v}}$ is a weighted average of the stock's beta estimated in the time-series and the average of all betas in the cross-section of month $t$, mean $_{C S}\left(\widehat{\beta_{\Pi, i, t}}\right)$. The former receives a larger weight when it is estimated more precisely, that is, when the square of the standard error of the estimated beta, $v r_{T S}$, is small relative to the cross-sectional variance of betas, $v r_{C S}$. From this point forward, inflation betas refer

\footnotetext{
${ }^{11}$ For example, Ang et al. (2007) write: "The motivation for the [ARMA(1,1)] model derives from a long tradition in rational expectations macroeconomics (see Hamilton, 1985) and finance (see Fama, 1975) that models inflation as the sum of expected inflation and noise. If expected inflation follows an $\mathrm{AR}(1)$ process, then the reduced-form model for inflation is given by an $\operatorname{ARMA}(1,1)$ model. The model also nicely fits the slowly decaying autocorrelogram of inflation."
} 
to the WLS and Vasicek-adjusted betas and we drop the hat and superscript $v$. Section 8. presents results for a variety of alternative specifications that test the robustness of these methodological choices.

\subsubsection{Inflation Portfolios}

Each month in our sample we create thirty value-weighted portfolios by two-way sorting all stocks at the intersection of ten inflation beta deciles and three size groups. The size groups are defined by the 20th and 50th percentile of NYSE market capitalization at the end of the previous month (the Micro, Small, and Big stocks of Fama and French, 2008). We then collapse the thirty portfolios into ten size-controlled inflation beta-sorted portfolios by averaging over the three size groups in each inflation beta decile. On the one hand, the smallest of stocks are illiquid, not in the set of stocks typically held by institutions that care most about inflation (such as pension funds), and their betas are harder to estimate. On the other hand, Ang, Brière, and Signori (2012) find that the best inflation hedgers in the CRSP file are the smallest stocks. To avoid favoring either hypothesis, we give equal weight to each size group in the inflation risk premium. Also, controlling for size in this way makes exposures to the market and other risk factors more homogeneous across portfolios. ${ }^{12}$ With a burn-in period of 60 months, this leaves us with a sample of post-ranking returns from July 1967 to December 2014.

\subsubsection{The Inflation Risk Premium}

We use three standard approaches to measure the inflation risk premium $\left(I P_{t}\right)$. The first estimate of the inflation risk premium is the difference in return between the High and Low inflation beta decile portfolio (denoted $\left.I P_{t}^{H L}\right)$.

The second estimate of the inflation risk premium is the return of a Breeden, Gibbons, and Litzenberger (1989) maximum correlation mimicking portfolio (denoted $I P_{t}^{M C}$ ). We construct this portfolio by projecting inflation innovations on the returns of the ten inflation beta-sorted portfolios, as they should contain a large share of the information in $u_{\Pi, t}$ that is relevant for the cross-section of stock returns. Table OA.1 of the Online Appendix presents the weights and confirms that the portfolios contain relevant inflation information.

Finally, each month we run a cross-sectional regression of individual stock returns on lagged inflation beta, controlling for market capitalization, book-to-market, and momentum (see, e.g., Fama and French, 2008; Chordia, Goyal, and Shanken, 2015). The time-series of estimated slope coefficients on lagged inflation beta represents our third estimate of the inflation risk premium (denoted $I P_{t}^{C S}$ ).

\subsubsection{Ex Post Inflation Exposures}

We present in Table 3 descriptive statistics for the set of ten inflation beta-sorted portfolios, as well as the three estimates of the inflation risk premium: $I P_{t}^{H L}, I P_{t}^{M C}$ and $I P_{t}^{C S}$. Panel A reports inflation exposures

\footnotetext{
${ }^{12}$ In Section 8., we show that our conclusions on the time-variation in inflation risk premia extend when we perform a single sort of stocks on inflation beta, without controlling for size.
} 
estimated with a simple OLS regression of portfolio returns on inflation innovations over the full sample. Analyzing whether these ex post exposures are large, economically and statistically, is important to asses whether inflation is a useless factor in the sense of Kan and Zhang (1999) and also serves as a reality check of the estimation procedure. We see that the ex post exposures line up almost monotonically from High to Low. The dispersion is wide, with a large post-ranking beta of 3.00 for $I P_{t}^{H L}$. A beta of three translates to an incremental monthly return of 76 basis points on average when $u_{\Pi, t}$ increases by one standard deviation. For comparison, the CRSP value-weighted market portfolio, with an inflation beta of -1.96 , loses 49 basis points on average for the same increase in $u_{\Pi, t}$. To accommodate comparison and interpretation, we have scaled the alternative measures of the inflation risk premium, $I P_{t}^{M C}$ and $I P_{t}^{C S}$, to have identical ex post inflation exposure to $I P_{t}^{H L}$. For these alternative estimates of the inflation risk premium, the ex post exposure is also strongly significant.

Fig. 2 plots the post-ranking inflation beta one month, one year, two years, five years, and ten years after sorting. To do so, we fix the portfolio composition at the sorting date $t$ and calculate monthly value-weighted returns up to ten years after. ${ }^{13}$ We then run a regression of monthly returns in $t+1, t+12, t+24, t+60$, and $t+120$ on contemporaneous (with the returns) innovations in inflation. In this way, we mimic the ex post monthly inflation exposure for an investor that rebalances infrequently with respect to inflation beta. We see that post-ranking inflation beta is increasing in pre-ranking beta up to ten years after the sort. The post-ranking beta for $I P_{t}^{H L}$ falls from 3.00 one month after sorting to a still large and significant 1.68 ten years after sorting. ${ }^{14}$ We conclude that our sort on ex ante inflation betas is powerful, because inflation betas are predictable many years ahead. Moreover, there is large cross-sectional dispersion in inflation risk, which is instrumental in identifying the inflation risk premium in the stock market.

We find that our ARMA(1,1)-inflation shocks are strongly correlated at 0.59 with first-differences in breakeven inflation, which is the difference between the yields of nominal and real ten year constant maturity treasury bonds (available from $F R E D^{\circledR}$ since 2003). Furthermore, Panel A of Table 4 shows that our sort (on exposures to $\operatorname{ARMA}(1,1)$-innovations in inflation) also creates large ex post exposures to first-differences in breakeven inflation, which we denote $\Delta B E_{t}$. The coefficient in a regression of returns of portfolios sorted on inflation beta on $\triangle B E_{t}$ increases monotonically in the inflation beta of the portfolios. The High-minusLow difference in $\triangle B E_{t}$-exposure is 9.79 with a $t$-statistic of 4.12 . In Panel $\mathrm{B}$, we sort individual stocks into deciles based on their exposure to $\Delta B E_{t}$. We see that ex post exposure to $\Delta B E_{t}$ increases monotonically in ex ante exposure. More importantly, the High-minus-Low difference in $\Delta B E_{t}$-exposure is not much larger than in Panel A at $12.47(t=4.82)$. Moreover, the correlation coefficient between the returns of the two

\footnotetext{
${ }^{13}$ When a stock leaves the sample, we reallocate its market cap to the remaining stocks in a given portfolio.

${ }^{14}$ This finding is seemingly inconsistent with the conclusion in Ang, Brière, and Signori (2012) that inflation betas are hard to estimate out-of-sample. However, their conclusion is based on a smaller sample of S\&P500 stocks from 1990 to 2009 . In the Appendix to their paper, the authors report results that are consistent with ours for a sort using all stocks in the CRSP universe from 1967 to 2009 .
} 
High-minus-Low portfolios is 0.86 , which suggests strong comovement between the two series.

\subsection{The Inflation Risk Premium Over Subsamples}

We present in Panels B and C of Table 3, respectively, the inflation risk premium over the full sample as well as in two subsamples split around 2002. This split is motivated by the fact that the nominal-real covariance, $N R C_{t}^{C}$, increased above its historical mean during 2002 , without falling below its mean again until the end of the sample (see Fig. 1). We present annualized average excess returns, Sharpe ratio, and CAPM alpha. We show in Section 8. that our main conclusions are robust to controlling for a broad set of benchmark asset pricing factors.

Over the full sample, average returns for the inflation beta-sorted portfolios are decreasing in inflation beta from $9.49 \%$ for Low to $5.26 \%$ for High. This dispersion translates to a marginally significant return for $I P_{t}^{H L}$ equal to $-4.23 \%(t=-2.08)$. This effect is similar in both magnitude and significance for $I P_{t}^{M C}$ and $I P_{t}^{C S}$ as well as in alpha. This unconditional inflation risk premium translates to a Sharpe ratio around -0.35 , which is in the same order of absolute magnitude as the Sharpe ratio of the aggregate stock market. The last column of the table presents the $p$-value from the monotonicity test of Patton and Timmermann (2010), where the null hypothesis is a non-decreasing relation of returns in inflation beta. For average returns, Sharpe ratios, and alphas, this null is marginally rejected.

Note that this relatively modest and only marginally significant unconditional estimate of the inflation risk premium does not imply that the inflation risk premium is economically unimportant. Rather, we argue that this unconditional estimate masks important variation over time that is driven by the nominal-real covariance and, in particular, its recent reversal. To see this, we show in Panel $\mathrm{C}$ that the inflation risk premium is considerably larger in the pre-2002 subsample, and strongly statistically significant. Average returns for $I P_{t}^{H L}, I P_{t}^{M C}$, and $I P_{t}^{C S}$ are $-7.49 \%,-5.85 \%$ and $-6.81 \%$, respectively, with $t$-statistics that all pass the data-mining corrected cutoff of three proposed in Harvey, Liu, and Zhu (2016). These average returns translate to a price of risk below -0.50 as measured by the Sharpe ratio, well above the aggregate stock market (in absolute value). Average returns are almost monotonically decreasing in inflation beta from $9.33 \%$ for the Low portfolio to $1.84 \%$ for the High portfolio. Consequently, the null of a non-decreasing relation is comfortably rejected at a $p$-value of 0.005 . We see similar results for CAPM alphas, suggesting that the one-factor CAPM does not price the cross-section of inflation portfolios in this subsample.

Post-2002, the inflation risk premium changes dramatically. Average returns are now increasing in inflation beta, resulting in an average return of $5.42 \%$ for the High-minus-Low portfolio and $3.49 \%$ for the cross-sectional regression portfolio. Although these estimates are non-negligible economically, they are not statistically significant over the short sample of twelve years. Moreover, the alternative estimate of the inflation risk premium, $I P_{t}^{M C}$, is virtually zero at $-0.06 \%$ and all three CAPM alphas are small and insignificant. Thus, the evidence suggests that the inflation risk premium is relatively small post-2002 and, if anything, 
positive, which is markedly different from the period pre-2002.

The last panel of the table presents the post-2002 minus pre-2002 differences. Average returns and alphas are monotonically decreasing from High to Low from $13.53 \%$ to $0.62 \%$ and $4.28 \%$ to $-5.13 \%$, respectively. Although the effects for $I P_{t}^{H L}, I P_{t}^{M C}$, and $I P_{t}^{C S}$ are not always significant, these differences are economically large and the monotonicity tests comfortably reject (at $p$-values below 0.01 ) the null of a non-increasing relation between the average or CAPM-adjusted (post-2002 minus pre-2002 difference in) return and inflation beta. Table OA.2 of the Online Appendix shows a slightly larger increase in the inflation risk premium when we split the sample in months where $N R C_{t}^{C}$ is above versus below its mean.

In short, we find that the inflation risk premium has increased markedly over time, consistent with the reversal in the nominal-real covariance from negative (on average since the 1960s) to positive (since the early 2000s).

\subsection{The Inflation Risk Premium Conditional on the Nominal-Real Covariance}

Because a sample split provides only a crude measure of the amount of time-variation in the inflation risk premium, we now turn to predictive regressions. We regress excess returns of the inflation portfolios (compounded over horizons $K$ of one, three, and twelve months) on $N R C_{t}^{C}$ using: $^{15}$

$$
R_{p, t+1: t+K}=L_{0}+L_{N R C} N R C_{t}^{C}+\varepsilon_{t+1: t+K}
$$

For each horizon, Panel A of Table 5 presents the estimated coefficients (annualized), both asymptotic NeweyWest (with $K$ lags) and bootstrapped (see Section 1 of the Online Appendix) $t$-statistics, and the adjusted $R^{2}$ for the individual decile portfolios and the three estimates of the inflation risk premium $\left(I P_{t}^{H L}, I P_{t}^{M C}\right.$, and $\left.I P_{t}^{C S}\right)$. We also present the $p$-values from Patton and Timmermann (2010) monotonicity tests. $N R C_{t}^{C}$ is standardized to have mean equal to zero and standard deviation equal to one to accommodate interpretation. Thus, $L_{0}$ measures the average excess return of the respective portfolio, that is, the unconditional inflation risk premium, whereas $L_{N R C}$ measures the increase in annualized portfolio return for a one standard deviation increase in the nominal-real covariance.

We find that the effect of $N R C_{t}^{C}$ on the inflation risk premium is positive, economically large and statistically significant (based on both asymptotic and bootstrap inference). For a one standard deviation increase in the nominal-real covariance, the inflation risk premium as estimated using $I P_{t}^{H L}, I P_{t}^{M C}$, and $I P_{t}^{C S}$ increases by about $3 \%$ to $4 \%$ for $K=1$. This increase is relative to an unconditional inflation risk premium of about $-4 \%$. The effect strengthens at longer horizons, growing to about $4 \%$ to $5 \%$ for $K=12$. The $R^{2}$ are also increasing in horizon, from around $0.5 \%$ for $K=1$ to over $6.5 \%$ for $K=12$. Finally, looking

\footnotetext{
${ }^{15}$ To see that these regressions use no forward-looking information, recall that $N R C_{t}^{C}$ is the slope coefficient $b_{t}^{12}$ in the regression of Eq. (2): $\Delta C_{s+1: s+12}=a_{t}^{12}+b_{t}^{12} \Pi_{s}+e_{s+1: s+12}$, with $s=1, \ldots, t-12$. Consistent with the fact that $b_{t}^{12}$ moves slowly over time, our results are insensitive to lagging the predictor by an additional month to control for a reporting delay in consumption data.
} 
at the decile portfolios, we see that $L_{N R C}$ is increasing monotonically from Low to High inflation beta, such that the null of non-monotonicity is comfortably rejected.

We conclude that the inflation risk premium is strongly time-varying with the nominal-real covariance. The economic intuition for this result is that high inflation beta stocks are attractive to hedge consumption risk when inflation predicts consumption with a negative sign as it did historically. However, these same stocks are not attractive as a hedge anymore, and could even expose investors to additional consumption risk, once the nominal-real covariance starts increasing, as it does towards the end of our sample, for instance. As a result, the returns of high versus low inflation beta stocks should be increasing in the nominal-real covariance, which is what we find.

Panel A of Table OA.3 of the Online Appendix shows that this conclusion is robust for two alternative measures of the nominal-real covariance, namely the time-varying relation between inflation and industrial production growth $\left(N R C_{t}^{I P}\right)$ and the negative of the stock market beta of a long-term treasury bond $\left(N R C_{t}^{-B B}\right)$. Each alternative measure predicts the inflation risk premium with a positive coefficient that is comparable in magnitude, significance, and horizon pattern to what we have seen before. Panel B of Table OA.3 shows that the nominal-real covariance predicts the inflation risk premium with a positive sign in both the first and second half of our sample period, although the predictive relation is stronger in the latter subsample. This result indicates that the time-variation in the inflation risk premium that we uncover is not exclusively driven by the recent reversal in the nominal-real covariance. Moreover, when we end our sample in 2007 , before interest rates reached the zero lower bound (ZLB), we find virtually identical effects to what we find in the full sample up to 2014. Thus, the time-variation that we uncover cannot be fully driven by asset pricing mechanisms that rely on interest rates at or close to the ZLB, such as those proposed in, for instance, Gourio and Ngo (2016), Bilal (2017) and Bretscher, Hsu, and Tamoni (2017).

\section{Origins of the Time-Varying Inflation Risk Premium}

To understand the drivers of the time-variation in the inflation risk premium, we next analyze (i) the relative contribution of the price versus quantities of inflation risk in our inflation beta-sorted decile portfolios, (ii) the importance of industry affiliation, and (iii) the relative contribution of cash flows versus discount rates to inflation exposure.

\subsection{Price and Quantities of Inflation Risk}

Fig. 3 shows the time variation in inflation beta for the High and Low decile portfolios, the difference between these two betas, and the average inflation beta of all ten portfolios. ${ }^{16}$ These inflation betas are estimated by applying Eqs. (4) and (5) to the post-ranking returns of each portfolio.

\footnotetext{
${ }^{16}$ The average beta is almost perfectly correlated (at 0.99 ) with the inflation beta of the CRSP value-weighted market portfolio.
} 
We see that there is considerable variation in inflation betas over time. For instance, the sudden increase in inflation betas around the turn of the century, consistent with the reversal in the nominal-real covariance, stands out. The correlation between the inflation beta of the High and Low portfolios is 0.93 , and both share a correlation of 0.98 with the cross-sectional average of all ten inflation betas. Moreover, although the High-minus-Low inflation beta is large and positive on average (and for most of the sample), the standard deviation of the High-minus-Low beta is much smaller than the standard deviation of either portfolio's beta in isolation (1.36 versus around 3.50). These results imply that time variation in the quantity of inflation risk is mostly common across portfolios (and in the stock market, more generally). This result is new to the literature and provides important information for our understanding of the inflation risk premium in the stock market.

To see this and quantify the relative contribution of quantities versus price of risk, we run pooled regressions of returns of the inflation beta-sorted decile portfolios, $p=H i g h, 2, \ldots$, Low on inflation beta $\left(\beta_{\Pi, p, t}\right)$, the nominal-real covariance $\left(N R C_{t}^{C}\right)$, and an interaction term:

$$
R_{p, t+1: t+K}=L_{0}+L_{\beta_{\Pi}} \beta_{\Pi, p, t}+L_{N R C} N R C_{t}^{C}+L_{\beta_{\Pi} \times N R C}\left(\beta_{\Pi, p, t} \times N R C_{t}^{C}\right)+\varepsilon_{p, t+1: t+K} .
$$

Panel A of Table 6 presents the estimated coefficients (annualized) and $t$-statistics using asymptotic standard errors calculated following Driscoll and Kraay (1998), which are robust to heteroscedasticity and rather general forms of cross-sectional and temporal dependence when the time dimension becomes large. We focus on the annual horizon $(K=12)$ and present consistent evidence for shorter horizons in Table OA.4 of the Online Appendix. To accommodate interpretation, $N R C_{t}^{C}$ is standardized in the time-series (as in Table 5) and $\beta_{\Pi, p, t}$ is demeaned in the pool, that is, its mean across portfolios and time is zero.

In column one, we do not include the interaction term ( $\left.\operatorname{set} L_{\beta_{\Pi} \times N R C}=0\right)$ and find that neither inflation beta nor the nominal-real covariance is a significant predictor of returns in the pool of inflation beta-sorted portfolios. The $R^{2}$ is also low at $0.71 \%$. In column two, we include the interaction term and see a large increase in the $R^{2}$ to $8.80 \%$. The estimated coefficient on the interaction term is large and significant at 2.36 $(t=3.63)$. This estimate indicates that expected returns are decreasing in inflation beta when the nominalreal covariance is negative, but increasing when it is positive. This result is in line with the large negative inflation risk premium we find in the subsample pre-2002, and the small positive inflation risk premium post-2002.

To further quantify the interaction effect between inflation beta and the nominal-real covariance, Panel B presents predicted risk premia in four distinct cases when: i) inflation beta is at plus or minus one (pooled) standard deviation from its mean and ii) the nominal-real covariance is at plus or minus one (timeseries) standard deviation from its mean. These predictions effectively evaluate the joint significance of the coefficients, thus alleviating concerns about correlation in the independent variables. We present both 
asymptotic and bootstrapped $t$-statistics. ${ }^{17}$

We find that the predicted risk premium is largest at $16.56 \%$ (with a bootstrapped $t$-statistic $>3$ ) for the case in which a portfolio has a low inflation beta and the nominal-real covariance is also low. This finding is intuitive: a portfolio of stocks is most risky when it comoves negatively with inflation shocks that contain bad news about future consumption growth. The predicted risk premium is also positive, but smaller at $7.65 \%\left(t_{\text {boot }}=1.65\right)$, when both inflation beta and the nominal-real covariance are high. When inflation beta is high (low) and the nominal-real covariance is low (high), predicted premia are close to zero. To interpret these predictions, note that the difference in expected returns between high and low inflation beta is positive when the nominal-real covariance is high and negative when the nominal-real covariance is low. This finding is consistent with the idea that the stocks that are most attractive to hedge high marginal utility states are those that have low (high) returns when inflation increases and the nominal-real covariance is positive (negative). This difference is largest in magnitude and significance when the nominal-real covariance is low, because in this case the covariance between inflation and future consumption growth is stronger in absolute terms (the nominal-real covariance at one standard deviation below the mean equals -2.24 , which is relative to -0.51 one standard deviation above the mean). ${ }^{18}$

We conclude that variation in inflation beta, that is, the quantity of inflation risk, contains important information about returns when combined with the nominal-real covariance. We further decompose inflation beta into two components: (i) a portfolio-specific component that captures cross-sectional variation in unconditional average inflation betas, $\widehat{\beta_{\Pi, p}}=T^{-1} \sum_{t=1}^{T} \beta_{\Pi, p, t}$; and, (ii) a time-specific component that captures time-series variation in the cross-sectional average inflation beta, $\widehat{\beta_{\Pi, t}}=10^{-1} \sum_{p=1}^{10} \beta_{\Pi, p, t}$.

In column three, we see that the interaction between the portfolio-specific component of inflation beta and the nominal-real covariance $\left(\widehat{\beta_{\Pi, p}} \times N R C_{t}^{C}\right)$ contains independent information about returns in the pool (relative to the individual effects of inflation beta and the nominal-real covariance). The coefficient estimate for the interaction term is $2.45(t=2.80)$. Setting the quantity of inflation risk equal to the unconditional spread in inflation beta between the High and Low inflation beta portfolios, a one standard deviation increase in the nominal-real covariance translates to a large and significant increase of $5.53 \%$ in the spread in expected returns between the High and Low portfolio $\left(5.53=\widehat{L_{\beta_{\Pi, p}}} \times N R C=\sigma_{N R C} \times\left(\widehat{\beta_{\Pi, H i g h}}-\widehat{\beta_{\Pi, L o w}}\right)=2.45 \times 1 \times 2.26\right.$, $\left.t_{\text {boot }}=2.17\right) .{ }^{19}$ The $R^{2}$ in column three is only $1.40 \%$, which implies that this specification still misses

\footnotetext{
${ }^{17}$ For details on the bootstrap procedure, see Section 1 of the Online Appendix.

${ }^{18}$ Although the magnitude of the difference between high and low inflation beta may seem large, at $-17.05 \%$ when the nominal-real covariance is low versus $10.98 \%$ when it is high, these effects are foreshadowed by the sorts we report in Table 3 . The difference between the High and Low inflation beta portfolio is -7.49 pre-2002 and $5.42 \%$ post-2002, and the 2002 cutoff only approximately splits the sample in low versus high nominal-real covariance months.

${ }^{19}$ This marginal effect is consistent with the regressions in Table 5 , which ask whether variation in the nominal-real covariance predicts High-minus-Low returns over time. The pooled specification in column three similarly asks whether the nominal-real covariance explains variation over time in the relative return of high versus low inflation beta portfolios, because the product $\widehat{\beta_{\Pi, p}} \times N R C_{t}^{C}$ varies monotonically from High to Low at each point in time due to the unconditional variation in inflation betas.
} 
variation in inflation betas that is relevant for the pool of inflation risk premia.

The missing variation is time-variation in the cross-sectional average inflation beta. To see this, column four shows that the interaction between the time-specific component of inflation betas and the nominal-real covariance $\left(\widehat{\beta_{\Pi, t}} \times N R C_{t}^{C}\right)$ contains relevant information about returns, given the large $R^{2}$ of $8.26 \%$ in this specification. The coefficient estimate is $2.45(t=3.45)$, which translates to a large and significant increase of $6.92 \%$ in the difference in expected return between periods of low (that is, the nominal-real covariance is one standard deviation below its mean) and average nominal-real covariance, when the cross-sectional average inflation beta decreases by one standard deviation $\left(6.92=L_{\widehat{\beta_{\Pi, t}}} \times N R C=-\sigma_{\widehat{\beta_{\Pi, t}}} \times-\sigma_{N R C}=2.45 \times-2.83 \times-1\right.$, $\left.t_{\text {boot }}=2.14\right)$. The intuition for this result is that at a time when the average inflation beta in the crosssection is low, the average stock is especially risky when the nominal-real covariance is negative (and a shock to inflation signals lower future growth).

In the last column of Panel A in Table 6, we include the two components of inflation beta (interacted with $N R C_{t}^{C}$ ) simultaneously. The $R^{2}$ equals $8.80 \%$, just like the model in column two, which includes the interaction term between not-decomposed inflation betas and the nominal-real covariance, $\beta_{\Pi, p, t} \times N R C_{t}^{C}$. This result suggests that the portfolio- and time-specific components of inflation betas together capture all the relevant variation in inflation betas for risk premia. ${ }^{20}$ Unconditional differences in the quantity of inflation risk, combined with the nominal-real covariance, drive the widening and compressing of the cross-section of inflation risk premia over time. With inflation betas held constant, this time-variation is thus driven by variation in the price of inflation risk. ${ }^{21}$ Time-variation in the average inflation beta, combined with the nominal-real covariance, determines the level of inflation risk premia in the cross-section. This last result suggests that the aggregate stock market risk premium contains an inflation component that is time-varying due to both the price and quantity of inflation risk.

To test this suggestion, we run time-series regressions of CRSP value-weighted market (excess) returns on the market's inflation beta, the nominal-real covariance, and an interaction term:

$$
R_{m, t+1: t+12}=L_{0, m}+L_{\beta_{\Pi}, m} \beta_{\Pi, m, t}+L_{N R C, m} N R C_{t}^{C}+L_{\beta_{\Pi} \times N R C, m}\left(\beta_{\Pi, m, t} \times N R C_{t}^{C}\right)+\varepsilon_{t+1: t+12},
$$

where $\beta_{\Pi, m, t}$ is estimated by regressing market returns on inflation innovations following Eq. (4). ${ }^{22}$ We present the results in Table 7 . To accommodate interpretation, $N R C_{t}^{C}$ is standardized and $\beta_{\Pi, m, t}$ is demeaned in the time-series (as in Table 6).

\footnotetext{
${ }^{20} \mathrm{By}$ implication, variation in inflation betas that is portfolio $\times$ time-specific, that is, variation that is not captured by a portfolio or a time fixed effect, contains no information for risk premia in the pool of inflation beta-sorted portfolios.

${ }^{21}$ Previous evidence in Table 5 is consistent with this conclusion. To see why, recall that the inflation risk premium estimated using cross-sectional regressions, $I P_{t}^{C S}$, represents the return of a portfolio strategy with an ex ante inflation beta fixed at one (Fama, 1976). Hence any variation in the returns of $I P_{t}^{C S}$ must follow from variation in the price of inflation risk.

${ }^{22}$ In Table OA.5 of the Online Appendix we present similar results when we proxy for the market portfolio using the average of the ten decile portfolios.
} 
In Panel A, we see that the market's inflation beta and the nominal-real covariance do not predict market returns in isolation. However, including their interaction term $\left(\beta_{\Pi, m, t} \times N R C_{t}^{C}\right)$ increases the $R^{2}$ from close to zero to $8.59 \%$. The coefficient estimate on the interaction term is economically large and significant at $2.85 \%(t=3.55)$. Similar to Panel B of Table 6 we also present the predicted market risk premium in four distinct cases. When both the nominal-real covariance and the market's inflation beta are low (that is, large and negative), stocks are most risky, and the predicted market risk premium is $13.61 \%(t=4.08)$, which is about double its unconditional mean. Furthermore, the difference in the predicted market risk premium when the market's inflation beta goes from low to high (i.e, from one standard deviation below the market's mean inflation beta to one standard deviation above) equals $6.65 \%$ when the nominal-real covariance is high but $-17.64 \%$ when the nominal-real covariance is low.

In Panel B, we present non-parametric estimates of the market risk premium, which are similar in absolute and relative magnitude to those reported in Panel A in all four cases. These estimates are calculated by averaging realized excess market returns in sample months $t+1$ when the market's inflation beta and the nominal-real covariance in month $t$ are either below the 40th or above the 60th percentile. In conclusion, the results in Table 7 confirm that the interaction between the market's inflation beta and the nominal-real covariance contains information about future market returns.

In Table OA.6 of the Online Appendix, we find that the interaction term between inflation beta and the nominal-real covariance, $\beta_{\Pi, p, t} \times N R C_{t}^{C}$, remains large and significant in a pooled regression that controls for market beta as well as the interaction between market beta and the nominal-real covariance. The same result obtains for the portfolio- and time-specific components of inflation betas. We conclude that variation in market betas does not fully explain the variation in risk premia we uncover.

\subsection{Industry Affiliation and Inflation Risk}

Having seen that inflation betas vary persistently and considerably over time and in the cross-section of stocks, we ask to what extent our results are driven by across-industry variation in inflation risk and returns. Among others, Boudoukh, Richardson, and Whitelaw (1994) and Ang, Brière, and Signori (2012) show that inflation betas vary across industries.

To start, we construct long-short industry factors using the top and bottom $K=1, \ldots, 5$ industries as ranked by their unconditional full sample exposure to innovations in inflation. Consistent with common intuition, Panel A of Table 8 shows that coal, oil, and gold are among the best unconditional inflation hedges while meals, textiles and clothes are among the worst. ${ }^{23}$ In every sample month, we then sort individual stocks into decile portfolios based on their rolling exposure to each industry factor, leaving out all stocks that belong to those top and bottom $K$ industries. In Panel B of Table 8, we first show that the High-minus-Low

\footnotetext{
${ }^{23}$ Table OA.7 of the Online Appendix shows that similar industries are also among the best (worst) conditional inflation hedges and that the industry decomposition is quite stable over time.
} 
returns from these sorts are significantly exposed ex post to $\operatorname{ARMA}(1,1)$-innovations in inflation for all $K$. In the remainder of Panel B, we see that the nominal-real covariance significantly predicts the returns of these High-minus-Low portfolios in the time-series, again for all $K$.

These results confirm that inflation betas vary across industries and that such variation contributes to time-variation in returns. However, these results do not fully separate the across-industry from the withinindustry contribution to the variation in inflation premia that we uncover in Section 4.. To isolate these within- and across-industry components, we calculate the inflation beta of each of the 48 industries from Kenneth French's website as the value-weighted average inflation beta of all stocks in that industry (estimated conditionally as in Eqs. (4) and (5)). We exclude industry-months that contain fewer than ten stocks. To obtain the across-industry component, we sort the industries into quintile portfolios, such that each acrossindustry portfolio contains nine or ten value-weighted industries. Using this sort, we calculate equal-weighted across-industry quintile returns. For the within-industry component, we first construct five value-weighted portfolios within each industry by splitting at the quintiles of ranked inflation betas. This gives us a total of 48-by-5 value-weighted portfolios. Within-industry quintile portfolio returns are then calculated as the equal-weighted average over the 48 industries. Table 9 presents the predictive regression of twelve-month compounded returns from each of these sorts on the nominal-real covariance. The last column presents the aggregate within-industry (across-industry) effect calculated as the difference between the High and Low within-industry (across-industry) inflation beta portfolio.

The unconditional inflation risk premium, as estimated by the intercept in the predictive regression, is negative for both the within- and across-industry sort. This unconditional premium is larger in magnitude across-industry (at $-3.36 \%$ with $t=-1.95$ ) than within-industry (at $-1.73 \%$ with $t=-1.55$ ). The coefficient estimate for the nominal-real covariance is positive, economically large and significant for both sorts at 4.63\% $(t=2.70)$ across-industry and 3.24\% $(t=3.29)$ within-industry. Both coefficient estimates follow from monotonic effects from High to Low inflation beta and translate to an $R^{2}$ that is slightly over $10 \%$. Monotonicity in the across-industry sort is important, because it suggests that our results are not solely driven by industries that are highly exposed to a particular component of aggregate inflation, such as oil. The within-industry result suggests that variation in inflation risk is priced in a time-varying way independent of the industry to which a stock belongs.

We conclude that although inflation risk certainly varies across industries, this variation is not crucial for generating the time-variation in inflation risk premia that we find.

\subsection{Inflation Risk in Cash Flows Versus Discount Rates}

In this section, we ask whether the cross-sectional variation in inflation risk in our portfolios is driven by cash flows or discount rates. We use the vector autoregression (VAR) approach of Campbell (1991) and Campbell and Vuolteenaho (2004) to decompose the unexpected component of inflation portfolios' monthly 
returns into discount rate and cash flow news, $R_{p, t+1}-E_{t}\left[R_{p, t+1}\right]=-N_{D R, t+1}+N_{C F, t+1}$. With this decomposition in hand, inflation beta, measured as the coefficient in a regression of $R_{p, t+1}-E_{t}\left[R_{p, t+1}\right]$ on $\operatorname{ARMA}(1,1)$-innovations in inflation $u_{\Pi, t+1}$, can be decomposed into a discount rate inflation beta plus a cash flow inflation beta:

$$
\begin{aligned}
\beta_{\Pi} & =\frac{\operatorname{Cov}\left(R_{p, t+1}-E_{t}\left[R_{p, t+1}\right], u_{\Pi, t+1}\right)}{\operatorname{Var}\left(u_{\Pi, t+1}\right)} \\
& =\frac{\operatorname{Cov}\left(-N_{D R, t+1}, u_{\Pi, t+1}\right)}{\operatorname{Var}\left(u_{\Pi, t+1}\right)}+\frac{\operatorname{Cov}\left(N_{C F, t+1}, u_{\Pi, t+1}\right)}{\operatorname{Var}\left(u_{\Pi, t+1}\right)} \\
& =\beta_{\Pi}^{-D R}+\beta_{\Pi}^{C F} .
\end{aligned}
$$

We consider three different specifications that include different state variables in the VAR. For the first specification, we use the state variables of Campbell and Vuolteenaho (2004), which are the the term spread, the market price-earnings ratio, and the value spread. For the second, we use the market dividend yield, default spread and term spread (following, e.g., Maio and Santa-Clara, 2012; Boons, 2016). For the third, we add the nominal-real covariance to the first specification. To ensure that the VAR models are correctly specified, we additionally include in all three specifications the dividend yield of the respective inflation beta-sorted portfolio as a state variable. ${ }^{24}$

Table 10 reports the inflation beta, $\beta_{\Pi}$, and its decomposition into $\beta_{\Pi}^{-D R}$ and $\beta_{\Pi}^{C F}$, for the High and Low inflation beta portfolios. The table also shows the share of the variance of $R_{p, t+1}-E_{t}\left[R_{p, t+1}\right]$ explained by $-N_{D R, t+1}, N_{C F, t+1}$, and twice their covariance. ${ }^{25}$ Consistent with the evidence discussed in Section 4.1.4., we find a large difference in inflation exposure in unexpected returns between the High and Low portfolios.

All three specifications have a High-minus-Low beta, $\beta_{\Pi, H i g h}-\beta_{\Pi, L o w}$, close to 1.8 . The discount rate component of beta, $\beta_{\Pi}^{-D R}$, is negative for the High and Low portfolios. In contrast, the cash flow component $\beta_{\Pi}^{C F}$ is positive for the High portfolio but negative for the Low one. It follows that in the inflation beta of the High-minus-Low portfolio, the discount rate component of the High and Low components offset each other, while the cash flow components reinforce each other. In the last row of the table, we present the fraction of the High-minus-Low inflation beta that can be attributed to exposure to cash flows, $\left(\beta_{\Pi, H i g h}^{C F}-\right.$ $\left.\beta_{\Pi, \text { Low }}^{C F}\right) /\left(\beta_{\Pi, \text { High }}-\beta_{\Pi, \text { Low }}\right)$. In specification two, this fraction equals $80 \%$. In specifications one and three, this fraction is above $100 \%$, which implies that the discount rate component actually reduces the spread in inflation betas in the cross-section of stocks. Thus, the evidence suggests that the inflation beta of the High-minus-Low portfolio is largely driven by exposure to cash flows. Importantly, this conclusion applies even though the relative contribution to the variance of returns (column labeled "\% Var") attributed to discount rate and cash flow news varies considerably across the three specifications.

\footnotetext{
${ }^{24}$ See Campbell and Ammer, 1993; Engsted, Pedersen, and Tanggaard, 2012.

${ }^{25}$ To reduce noise, the High and Low portfolios in this section are top and bottom terciles. These terciles simply average returns over the top and bottom three deciles of our original inflation beta-sorted portfolios.
} 


\section{Model}

To motivate our empirical findings, we model the processes for consumption, inflation and the nominal-real covariance as exogenous, generating the time-varying predictability of consumption with inflation uncovered in the data in Section 3.. The model then produces - via pricing through the Euler equation of a representative agent with Epstein-Zin utility - equilibrium quantities of inflation risk for the cross-section of stocks and the aggregate market, together with a market price of inflation risk. These quantities and prices behave qualitatively and quantitatively like the ones we find empirically in Sections 4 . to $5 .{ }^{26}$

\subsection{Exogenous Processes and Stochastic Discount Factor}

The exogenous processes for real consumption growth $\left(\Delta c_{t+1}\right)$, inflation $\left(\pi_{t+1}\right)$, the nominal-real covariance $\left(\varphi_{t+1}\right)$, and real dividend growth for asset $i\left(\Delta d_{i, t+1}\right)$ are given by

$$
\begin{aligned}
\pi_{t+1} & =\mu_{\pi}+\rho_{\pi}\left(\pi_{t}-\mu_{\pi}\right)+\xi_{\pi} u_{t}+\phi_{\pi} u_{t+1}, \\
\Delta c_{t+1} & =\mu_{c}+\rho_{c}\left(\pi_{t}-\mu_{\pi}\right)+\xi_{c} \varphi_{t-1} u_{t}+\sigma_{c} \eta_{t+1}, \\
\varphi_{t+1} & =\varphi_{0}+v\left(\varphi_{t}-\varphi_{0}\right)+\sigma_{w} w_{t+1}, \\
\Delta d_{i, t+1} & =\mu_{i}+\rho_{i}\left(\pi_{t}-\mu_{\pi}\right)+\xi_{i} \varphi_{t-1} u_{t}+\sigma_{i} \eta_{t+1}, \\
u_{t}, \eta_{t}, w_{t} & \sim i i d \mathcal{N}(0,1) .
\end{aligned}
$$

Consistent with our empirical analysis, inflation in Eq. (13) is an $\operatorname{ARMA}(1,1)$-process with innovation $u_{\Pi, t+1} \equiv \phi_{\pi} u_{t+1}$. Inflation and inflation shocks affect the real economy via expected consumption growth in Eq. (14). Inflation predicts consumption growth unconditionally and in a time-invariant way with sign and magnitude determined by $\rho_{c}$. In contrast, the inflation shock $u_{t}$ predicts consumption growth in a timevarying manner, where the sign and magnitude of the predictability are given by $\xi_{c} \varphi_{t-1}$. The nominal-real covariance, $\varphi_{t}$, follows the mean-reverting process given in Eq. (15) and can change sign over time. Dividends in Eq. (16) are subject to the same risks as consumption, but with potentially different exposures. The shocks $u_{t+1}, \eta_{t+1}$ and $w_{t+1}$ are i.i.d. standard normal.

The representative agent has Epstein-Zin utility, which yields the (log) stochastic discount factor (SDF)

$$
m_{t+1}=\theta \log \delta-\frac{\theta}{\psi} \Delta c_{t+1}-(1-\theta) r_{c, t+1}
$$

where $r_{c, t+1}$ is the $(\log )$ return on the wealth portfolio, that is, the claim on aggregate consumption; $\delta \in(0,1)$ is the discount rate; $\psi>0$ is the elasticity of intertemporal substitution (EIS); and $\theta=(1-\gamma) /(1-1 / \psi)$, with $\gamma>0$ the coefficient of relative risk aversion.

\footnotetext{
${ }^{26}$ Detailed derivations for all the results presented in this section can be found in Section 2 of the Online Appendix.
} 


\subsection{The Nominal-Real Covariance}

The nominal-real covariance, $\varphi_{t}$, plays a dual role in our model. It determines $(i)$ the time variation in the predictability of consumption with inflation and (ii) the stochastic volatility of expected consumption growth.

To see that the nominal-real covariance determines the time variation in the predictability of consumption, we run in the model the same two-stage procedure that we run in the data in Eqs. (2) and (3). The modelimplied coefficient on inflation in the first-stage regression (2) is

$$
b_{t}^{K}=\frac{\operatorname{Cov}_{t}\left(\sum_{j=1}^{K} \Delta c_{t+1+j}, \pi_{t+1}\right)}{\operatorname{Var}_{t}\left(\pi_{t+1}\right)}=\rho_{c} h_{K}+\frac{\xi_{c}}{\phi_{\pi}} \varphi_{t},
$$

where $h_{K}$ is a constant that depends on the prediction horizon $K$ and parameters of the inflation process. ${ }^{27}$ If $\xi_{c} \neq 0$, Eq. (19) shows that inflation predicts cumulative consumption growth in a time varying manner determined by $\varphi_{t}$. Eq. (19) clarifies why we call $\varphi_{t}$ the nominal-real covariance. Under the null of the model, $\varphi_{t}$ is a linear transformation of $\widehat{b_{t}^{12}}=N R C_{t}^{C}$, the same variable we use in the data to measure the nominal-real covariance. The second stage regression in Eq. (3) also holds exactly in the model with $d_{0}^{c, K}=0$ and $d_{1}^{c, K}=1$ (for any $K$ and any calibration of the model).

To see that the nominal-real covariance also plays the role of stochastic volatility of expected consumption growth, it is instructive to compare our model to the long-run risk model of Bansal and Yaron (2004). Replacing $\pi_{t}-\mu_{\pi}$ with long-run risk and setting $\xi_{\pi}=\xi_{c}=\xi_{i}=0$, we recover the basic version of the longrun risk model that does not have stochastic volatility. Thus, the new elements in our model, in addition to considering inflation instead of long-run risk, are the terms $\xi_{\pi} u_{t}, \xi_{c} \varphi_{t-1} u_{t}$ and $\xi_{i} \varphi_{t-1} u_{t}$ in Eqs. (13), (14) and (16). In the version of the long-run risk model with stochastic volatility, there is a single mean-reverting process that drives the stochastic volatilities of both expected and unexpected consumption growth (where we recall that long-run risk is exactly equal to expected consumption growth). In our model, we have stochastic volatility for neither inflation nor unexpected consumption growth, but we do have stochastic volatility for expected consumption growth through the term $\xi_{c} \varphi_{t-1} u_{t}{ }^{28}$

\footnotetext{
${ }^{27}$ Using changes in inflation $\left(\pi_{t+1}-\pi_{t}\right)$ or inflation innovations $\left(u_{\Pi, t+1}\right)$ instead of inflation in Eq. (19) gives $b_{t}^{K}$ that are also afine in $\varphi_{t}$. When standardized to have mean zero and variance one, these different measures of the nominal-real covariance are identical. Empirically, using inflation shocks (for instance, from an ARMA(1,1)-process) instead of inflation $\left(\Pi_{t}\right)$ in Eqs. (2) and (3) gives similar estimates of the nominal-real covariance $b_{t}^{12}$ with correlations above $90 \%$.

${ }^{28} \mathrm{An}$ additional advantage of modeling $\varphi_{t}$ (instead of $\varphi_{t}^{2}$ as in the long-run risk literature) as an $A R(1)$ process is that volatilities are guaranteed to always be positive.
} 


\subsection{The Price of Inflation Risk}

To understand the asset pricing implications of the model, we study innovations in the SDF by using a decomposition similar to that in Campbell, Giglio, Polk, and Turley (2018):

$$
\begin{aligned}
m_{t+1}-E_{t} m_{t+1} & =-\gamma\left(E_{t+1}-E_{t}\right) \Delta c_{t+1}-(1-\theta) \kappa_{1}\left(E_{t+1}-E_{t}\right) w c_{t+1} \\
& =-\gamma N_{C, t+1}-(1-\theta) \kappa_{1}\left(\left(1-\frac{1}{\psi}\right) N_{P A T H, t+1}+\frac{\theta \kappa_{1}^{2}}{2} N_{R I S K, t+1}\right)
\end{aligned}
$$

where we define

$$
\begin{aligned}
N_{C, t+1} & \equiv\left(E_{t+1}-E_{t}\right) \Delta c_{t+1}, \\
N_{P A T H, t+1} & \equiv\left(E_{t+1}-E_{t}\right) \sum_{j=0}^{\infty} \kappa_{1}^{j} E_{t+1+j}\left[\Delta c_{t+2+j}\right], \\
N_{R I S K, t+1} & \equiv\left(E_{t+1}-E_{t}\right) \sum_{j=0}^{\infty} \kappa_{1}^{j} \operatorname{Var}_{t+1+j}\left(w c_{t+2+j}\right),
\end{aligned}
$$

and $\kappa_{1}$ is an approximation constant generally close to one. Eq. (20) decomposes news to the SDF into news to contemporaneous consumption growth $\left(N_{C, t+1}=\left(E_{t+1}-E_{t}\right) \Delta c_{t+1}\right)$ and news to the (log) wealth consumption ratio $\left(\left(E_{t+1}-E_{t}\right) w c_{t+1}\right)$. Eq. (21) further decomposes news to the wealth consumption ratio into $N_{P A T H, t+1}$, which is news about the future expected path of consumption, and $N_{R I S K, t+1}$, which is "news about risk" (revisions in the future conditional variance of the wealth-consumption ratio).

Using Eq. (14), news to contemporaneous consumption growth can be written as $N_{C, t+1}=\sigma_{c} \eta_{t+1}$, which shows that $N_{C, t+1}$ depends on the consumption shock $\eta_{t+1}$, but not on any of the other shocks. $N_{C, t+1}$ enters the SDF by standard consumption-CAPM logic. $N_{P A T H, t+1}$ and $N_{R I S K, t+1}$ enter the SDF because with non-time separable utility, today's marginal utility depends not only on contemporaneous consumption growth but also on its entire future path. As can be seen directly from their definition, $N_{P A T H, t+1}$ and $N_{R I S K, t+1}$ depend only on future consumption growth and not on contemporaneous consumption growth, so they do not depend on the consumption shock $\eta_{t+1}$. It follows that news to the wealth-consumption ratio arises not from changes in contemporaneous consumption but exclusively from changes in wealth, that is, from changes in the pricing of the aggregate consumption claim.

When the EIS is greater than one, which we assume, the intertemporal substitution effect dominates the wealth effect. Positive news to the expected path of consumption growth $\left(N_{P A T H, t+1}>0\right)$ leads the representative agent to invest more in the aggregate wealth portfolio, increasing its price and hence also the wealth-consumption ratio. $N_{P A T H, t+1}$ depends only on the inflation shock, $u_{\Pi, t+1}\left(=\phi_{\pi} u_{t+1}\right)$, because by Eq. (14) it is the only shock that provides news about expected consumption growth (shocks to the nominal-real covariance that affect expected consumption growth at $t$ arrive at $t-1$, so they provide no news). Using Eq. (13), we find that

$$
N_{P A T H, t+1}=\left(\frac{\rho_{c} \phi_{\pi}}{1-\rho_{\pi} \kappa_{1}}+\frac{\rho_{c} \kappa_{1} \xi_{\pi}}{1-\rho_{\pi} \kappa_{1}}+\xi_{c} \varphi_{t}\right) \frac{u_{\Pi, t+1}}{\phi_{\pi}}
$$


Eq. (22) will be key to understanding the inflation risk premium. The first two terms inside the parenthesis in Eq. (22) arise through the term $\rho_{c}\left(\pi_{t}-\mu_{\pi}\right)$ in Eq. (14). The first term reflects that news to inflation is news to expected consumption growth. The second term reflects a more indirect channel: in Eq. (13), $u_{t}$ predicts future inflation $\pi_{t+1}$, which in turn predicts expected consumption growth one period later. Both of these effects reflect the unconditional (non-time-varying) effect of inflation on consumption and are stronger when inflation has a larger or longer-lasting impact on future consumption, which is when inflation is more persistent (higher $\rho_{\pi}$ ), more volatile (higher $\phi_{\pi}$ or $\xi_{\pi}$ ), or more predictive of consumption (higher $\rho_{c}$ ).

The third term in Eq. (22) reflects the conditional (time-varying) relation between consumption and inflation. It appears because the term $\xi_{c} \varphi_{t-1} u_{t}$ in Eq. (14) is part of expected consumption growth. Inflation shocks can be good or bad news for the expected path of consumption. When $\xi_{c} / \phi_{\pi}>0$, a positive inflation shock $\left(u_{\Pi, t+1}>0\right)$ is good news for the path of expected consumption if and only if $\varphi_{t}>0$, just as in the data and our model calibration below. The volatility of $N_{P A T H, t+1}$ increases when $\xi_{c}$ is higher and when $\varphi_{t}$ is more volatile (higher $\sigma_{w}$ ). The persistence of $N_{P A T H, t+1}$ increases when $\varphi_{t}$ is more persistent (higher $v$ ).

When the representative agent has a preference for early resolution of uncertainty $(\theta<0)$, which we assume, news about risk increasing $\left(N_{R I S K, t+1}>0\right)$ represents a bad state of nature. Even though the expected path of consumption growth remains unchanged, the consumption claim has become riskier. In equilibrium, today's price of the wealth portfolio must go down for the representative agent to be willing to hold the riskier consumption claim, which results in a lower wealth-consumption ratio and a higher marginal utility. $N_{R I S K, t+1}$ depends only on the nominal-real covariance shock $w_{t+1}$, because $N_{R I S K, t+1}$ is a function of conditional second moments only and the nominal-real covariance is the only source of heteroskedasticity.

Because each shock $\eta_{t+1}, u_{\Pi, t+1}$, and $w_{t+1}$ drives a separate news component of the SDF, we can give an intuitive interpretation for the price of risk for each of them. Innovations to the SDF as a function of shocks and their respective prices of risk are

$$
m_{t+1}-E_{t}\left[m_{t+1}\right]=-\lambda_{\eta} \eta_{t+1}-\lambda_{u, t} u_{\Pi, t+1}-\lambda_{w, t} w_{t+1}-\lambda_{2 w}\left(\frac{w_{t+1}^{2}-1}{\sqrt{2}}\right)
$$

where the shocks $\eta_{t+1}, u_{\Pi, t+1}, w_{t+1}$, and $\left(w_{t+1}^{2}-1\right) / \sqrt{2}$ are all mean zero and uncorrelated (but not independent) from each other.

Comparing to Eq. (21), and using Eq. (22), we can identify the price of inflation risk $\lambda_{u, t}$ in

$$
\lambda_{u, t} u_{\Pi, t+1}=\kappa_{1}\left(\gamma-\frac{1}{\psi}\right) N_{P A T H, t+1}=\kappa_{1}\left(\gamma-\frac{1}{\psi}\right)\left(\frac{\rho_{c}\left(\phi_{\pi}+\kappa_{1} \xi_{\pi}\right)}{1-\rho_{\pi} \kappa_{1}}+\xi_{c} \varphi_{t}\right) \frac{u_{\Pi, t+1}}{\phi_{\pi}} .
$$

The price of inflation risk $\lambda_{u, t}$ is, by definition, the compensation that the representative agent requires to hold one unit of inflation risk $\left(u_{\Pi, t+1}\right.$-risk). Our model provides the economic interpretation that inflation risk is priced because inflation shocks provide news to the expected future path of consumption growth, and changes in this path change the marginal utility of the representative agent. In addition, the model provides 
an equilibrium explanation for why the price of inflation risk moves over time together with the nominal real covariance. When $\varphi_{t}>0$, the representative agent requires positive compensation for bearing inflation risk, because positive inflation shocks contain good news about future consumption growth. Since higher future consumption growth is a good state of nature, inflation has a positive covariance with marginal utility. On the other hand, if $\varphi_{t}<0$, the representative agent is willing to accept lower expected returns to hold inflation risk, because inflation shocks come with bad news about future consumption growth, making inflation a good consumption and marginal utility hedge. Since $\lambda_{u, t}$ is proportional to $N_{P A T H, t+1}$, its dependence on parameters is the same as for $N_{P A T H, t+1}$, already discussed above. In addition to $N_{P A T H, t+1}, \lambda_{u, t}$ also depends on preference parameters. In particular, because inflation shocks generate risk over the entire future path of expected consumption growth, a stronger preference for early resolution of uncertainty (higher $\gamma$ or $\psi)$ magnifies the effect of news about the expected path of consumption on the price of inflation risk.

The same logic that we used for inflation can be used to find the prices of risk for consumption and nominal-real covariance shocks and to interpret them, respectively, as coming from news to contemporaneous consumption, $N_{C, t+1}$, and from news about risk, $N_{R I S K, t+1}$. Nominal-real covariance risk $\left(w_{t}\right.$-risk) is priced because of both of the roles that the nominal-real covariance plays in this model (it controls the time variation in the predictability of consumption with inflation and it also drives the stochastic volatility of expected consumption growth). However, the nominal-real covariance interacts with inflation risk $\left(u_{\Pi, t}-\mathrm{risk}\right)$ only due to its role in the predictability of consumption with inflation, and not because of its role in inducing stochastic volatility. ${ }^{29}$

\subsection{The Quantity of Inflation Risk and Inflation Betas}

A Campbell-Shiller approximation implies that $(\log )$ returns for asset $i, r_{i, t+1}$, can be written as

$$
r_{i, t+1}=\kappa_{i, 0}+\kappa_{i, 1} p d_{i, t+1}-p d_{i, t}+\Delta d_{i, t+1}
$$

where $p d_{i, t}$ is the $(\log )$ price-dividend ratio of $i$, and $\kappa_{i, 0}, \kappa_{i, 1}$ are approximation constants with $\kappa_{i, 1}$ generally close to one. ${ }^{30}$ Decomposing news to returns in a manner similar to the SDF, we have

$$
\begin{aligned}
\left(E_{t+1}-E_{t}\right) r_{i, t+1} & =\kappa_{i, 1}\left(E_{t+1}-E_{t}\right) p d_{i, t+1}+\left(E_{t+1}-E_{t}\right) \Delta d_{i, t+1} \\
& =\kappa_{i, 1}\left(N_{P A T H, t+1}^{i}+N_{m, t+1}^{i}+\frac{1}{2} N_{R I S K, t+1}^{i}\right)+N_{D, t+1}^{i},
\end{aligned}
$$

\footnotetext{
${ }^{29}$ Section 2 of the Online Appendix has expressions and a deeper discussion of consumption and nominal-real covariance risks, which we omit here since our main focus is on inflation risk.

${ }^{30}$ Eq. (24) and all formulas below apply also to the consumption portfolio (with $i=c$ ) if we replace $p d_{i, t}, \Delta d_{i, t}, \kappa_{i, 0}$ and $\kappa_{i, 1}$ with $w c_{t}, \Delta c_{t}, \kappa_{0}$ and $\kappa_{1}$, respectively.
} 
where

$$
\begin{aligned}
N_{P A T H, t+1}^{i} & \equiv\left(E_{t+1}-E_{t}\right) \sum_{q=0}^{\infty} \kappa_{i, 1}^{q} E_{t+1+q}\left[\Delta d_{i, t+2+q}\right], \\
N_{m, t+1}^{i} & \equiv\left(E_{t+1}-E_{t}\right) \sum_{q=0}^{\infty} \kappa_{i, 1}^{q} E_{t+1+q}\left[m_{t+2+q}\right], \\
N_{R I S K, t+1}^{i} & \equiv\left(E_{t+1}-E_{t}\right) \sum_{q=0}^{\infty} \kappa_{i, 1}^{q} \operatorname{Var}_{t+1+q}\left[m_{t+2+q}+\kappa_{i, 1} p d_{i, t+1}+\Delta d_{i, t+1}\right], \\
N_{D, t+1}^{i} & \equiv\left(E_{t+1}-E_{t}\right) \Delta d_{i, t+1} .
\end{aligned}
$$

Using the same line of reasoning used to interpret Eq. (21), we find that $N_{P A T H, t+1}^{i}$, which is news to the expected future path of dividends of asset $i$, depends only on the inflation shock $u_{\Pi, t+1} ; N_{R I S K, t+1}^{i}$, which is news to the conditional future variance of $m_{t+1}+\kappa_{i, 1} p d_{i, t+1}+\Delta d_{i, t+1}$, depends only on the nominal-real covariance shock $w_{t+1}$; and $N_{D, t+1}^{i}$, which is news to contemporaneous dividend growth of asset $i$, depends only on the consumption shock $\eta_{t+1}$. In addition to these three news components, news to the returns of asset $i$ also depends on $N_{m, t+1}^{i}$, which is news to the expected path of the SDF (news to expected discount rates). This news depends on the inflation shock, $u_{\Pi, t+1}$, and the nominal-real covariance shock, $w_{t+1}$. In news for returns, $N_{m, t+1}^{i}$ enters because positive news to the future expected path of the SDF $\left(N_{m, t+1}^{i}>0\right)$ is positive news for today's returns: future marginal utility is expected to be higher, so the same stream of dividends is more valuable, pushing up the price-dividend ratio and realized returns. ${ }^{31}$

The quantity of inflation risk of asset $i$ is given by its inflation beta. As in our empirical analysis, the inflation beta $\beta_{\Pi, i, t}$ is defined as the coefficient in a regression of returns, $r_{i, t+1}$, on inflation shocks, $u_{\Pi, t+1}$, conditional on time $t$ information. Using Eq. (25), we have

$$
\beta_{\Pi, i, t} \equiv \frac{\operatorname{Cov}_{t}\left(u_{\Pi, t+1}, r_{i, t+1}\right)}{\operatorname{Var}_{t}\left(u_{t+1}\right)}=\kappa_{i, 1} \operatorname{Cov}_{t}\left(u_{\Pi, t+1}, N_{m, t+1}^{i}\right)+\kappa_{i, 1} \operatorname{Cov}_{t}\left(u_{\Pi, t+1}, N_{P A T H, t+1}^{i}\right) .
$$

Intuitively, when news to the path of either dividends, $N_{P A T H, t+1}^{i}$, or discount rates, $N_{m, t+1}^{i}$, covary more with inflation shocks, the price of asset $i$ is more exposed to inflation and the quantity of inflation risk increases. The reason that news to only expected dividends and expected discount rates enter $\beta_{\Pi, i, t}$ is that inflation shocks only affect the expected paths of dividends and discount rates, but not their higher (conditional) moments. $N_{R I S K, t+1}^{i}$ and $N_{D, t+1}^{i}$ are uncorrelated to inflation shocks $u_{\Pi, t+1}$, so they do not contribute to $\beta_{\Pi, i, t}$.

\footnotetext{
${ }^{31}$ Given that the risk free rate is defined by $r_{f, t}=-E_{t}\left[m_{t+1}\right]-\frac{1}{2} \operatorname{Var} t\left(m_{t+1}\right)$, this news component is related to, but not the same as, news to the path of risk-free rate. $N_{m, t+1}^{i}$ is different for different assets because the expected path of the SDF is discounted by the asset specific constant $\kappa_{i, 1}$.
} 
Computing the covariances in Eq. (26), we find that ${ }^{32}$

$$
\beta_{\Pi, i, t}=-\kappa_{i, 1} \underbrace{\frac{\phi_{\pi}}{\psi}\left(\frac{\rho_{c}\left(\kappa_{i, 1} \xi_{\pi}+\phi_{\pi}\right)}{1-\kappa_{i, 1} \rho_{\pi}}+\xi_{c} \varphi_{t}\right)}_{\operatorname{Cov}_{t}\left(u_{\Pi, t+1}, N_{m, t+1}^{i}\right)}+\kappa_{i, 1} \underbrace{\phi_{\pi}\left(\frac{\rho_{i}\left(\kappa_{i, 1} \xi_{\pi}+\phi_{\pi}\right)}{1-\kappa_{i, 1} \rho_{\pi}}+\xi_{i} \varphi_{t}\right)}_{\operatorname{Cov}_{t}\left(u_{\Pi, t+1}, N_{P A T H, t+1}^{i}\right)} .
$$

The intuition behind the dependence of $\beta_{\Pi, i, t}$ on preference parameters and parameters of the exogenous processes in Eqs. (13) to (16) is similar to the intuition for the terms in Eq. (23) discussed earlier. More importantly, all time variation in $\beta_{\Pi, i, t}$ comes from $\varphi_{t}$.

\subsection{Risk Premia of Inflation Beta-Sorted Portfolios}

The risk premium on asset $i$ is

$$
-\operatorname{Cov}_{t}\left(m_{t+1}, r_{i, t+1}\right)=\lambda_{u, t} \beta_{\Pi, i, t}+\lambda_{w, t} \beta_{w, i, t}+\lambda_{2 w} \beta_{2 w, i}+\lambda_{\eta} \beta_{\eta, i},
$$

where the inflation risk premium component is

$$
\lambda_{u, t} \beta_{\Pi, i, t}=\underbrace{\kappa_{1}\left(\gamma-\frac{1}{\psi}\right)\left(\frac{\phi_{\pi}+\kappa_{1} \xi_{\pi}}{1-\kappa_{1} \rho_{\pi}} \rho_{c}+\xi_{c} \varphi_{t}\right)}_{\lambda_{u, t}} \underbrace{\kappa_{i, 1} \phi_{\pi}\left(\frac{\phi_{\pi}+\kappa_{i, 1} \xi_{\pi}}{1-\kappa_{i, 1} \rho_{\pi}}\left(\rho_{i}-\frac{\rho_{c}}{\psi}\right)+\left(\xi_{i}-\frac{\xi_{c}}{\psi}\right) \varphi_{t}\right)}_{\beta_{\Pi, i, t}} .
$$

Because the price of inflation risk, $\lambda_{u, t}$, and the quantity of inflation risk, $\beta_{\Pi, i, t}$, are each linear functions of the nominal real covariance, the inflation risk premium is quadratic in $\varphi_{t}$. The inflation risk premium is positive when $\lambda_{u, t}$ and $\beta_{\Pi, i, t}$ have the same sign. If $\lambda_{u, t}$ and $\beta_{\Pi, i, t}$ are both negative, the returns of asset $i$ comove negatively with inflation shocks that contain bad news for future growth. If $\lambda_{u, t}$ and $\beta_{\Pi, i, t}$ are both positive, the returns of asset $i$ comove positively with inflation shocks that contain good news. In both cases, the payoff of asset $i$ is low in bad times and high in good times, exacerbating consumption risk. In order for the representative agent to be willing to hold asset $i$ in equilibrium, the inflation risk premium must be positive. If $\lambda_{u, t}$ and $\beta_{\Pi, i, t}$ have different signs, the opposite is true. Asset $i$ has high payoff in bad states and low payoff in good states, and the representative agent is willing to accept a negative premium to hold this asset because it provides a consumption hedge.

To derive the model-implied inflation risk premium from the cross-section of stock returns, we model two assets that represent the High $(H)$ and Low $(L)$ inflation beta portfolios from our empirical analysis. The High-minus-Low inflation beta, $\beta_{u, H L, t}$, is simply the difference $\beta_{u, H t}-\beta_{u, L t}$, because betas are linear in returns (see Eq. (26)). If $\kappa_{H, 1} \approx \kappa_{L, 1}$, as is the case in any reasonable calibration of the model, a good

\footnotetext{
${ }^{32}$ Using changes in inflation $\left(\pi_{t+1}-\pi_{t}\right)$ or inflation levels $\left(\pi_{t+1}\right)$ instead of inflation innovations in Eq. (26) also give $\beta_{\Pi, i, t}$ that are afine in $\varphi_{t}$ and differ from each other only in scaling. Thus, as in the data (see Section 8 . and Table OA.13 in the Online Appendix), these different ways to measure inflation risk capture the same time-variation driven by the nominal-real covariance.
} 
approximation for $\beta_{u, H L, t}$ is

$$
\begin{aligned}
\beta_{u, H L, t} & =\operatorname{Cov}_{t}\left(u_{\Pi, t+1}, N_{P A T H, t+1}^{H}\right)-\operatorname{Cov}_{t}\left(u_{\Pi, t+1}, N_{P A T H, t+1}^{L}\right) \\
& =\frac{\phi_{\pi}+\xi_{\pi}}{1-\rho_{\pi}}\left(\rho_{H}-\rho_{L}\right)+\left(\xi_{H}-\xi_{L}\right) \varphi_{t}
\end{aligned}
$$

with associated inflation risk premium $\lambda_{u, t} \beta_{u, H L, t}=\lambda_{u, t}\left(\beta_{u, H t}-\beta_{u, L t}\right)$. The High-minus-Low inflation beta is determined by how differently inflation shocks covary with news to the expected path of dividends for asset $H$ versus asset $L$. The assumption that $\kappa_{H, 1} \approx \kappa_{L, 1}$ makes the discount-rate components $\kappa_{H, 1} \operatorname{Cov}_{t}\left(u_{\Pi, t+1}, N_{m, t+1}^{H}\right)$ and $\kappa_{L, 1} \operatorname{Cov}_{t}\left(u_{\Pi, t+1}, N_{m, t+1}^{L}\right)$ of $\beta_{u, H, t}$ and $\beta_{u, L, t}$ identical, so they cancel out when taking the difference.

\section{Calibration}

Table 11 shows the calibrated parameters of the model and Table 12 shows the empirical and modelimplied moments that we target. ${ }^{33}$ The model has 26 parameters ( 3 preference parameters; 11 parameters for the joint process of inflation, consumption and the nominal-real covariance; and 4 parameters for each of the three stocks we model) and we consider 37 moments from Tables 1 to 7.

For preferences, we use $\delta=0.99, \psi=2$ and $\gamma=15$, which are within the range of values commonly used in similar studies. ${ }^{34}$ We calibrate the exogenous processes for inflation, consumption and the nominal-real covariance in Eqs. (13) to (15) to match the summary statistics of Tables 1 and the predictability regressions of Table 2 for $K=12$. The model-implied moments for $\pi_{t}$ and $\Delta c_{t}$ match the data well. The one exception is the standard deviation of $\Delta c_{t}$, which at $2.49 \%$ is somewhat higher than the $1.13 \%$ in the data. This value is within the range of values used in other studies, however. ${ }^{35}$ The relatively high volatility of $\Delta c_{t}$ is needed to match the relatively high volatility and persistence of the nominal-real covariance under the parsimonious $A R(1)$ specification for $\varphi_{t}$. We prioritize matching moments of the nominal-real covariance, because that is the mechanism we propose to explain the time-variation in the inflation risk premium. In any case, by standard consumption-CAPM logic, whether the standard deviation of consumption is $1.13 \%$ or $2.49 \%$ has little impact on asset prices, even with $\gamma=15$. The $A R(1)$ coefficient for $\Delta c_{t}$ is essentially zero in the model and small (point estimate -16.84\%) and not statistically significant in the data at the $1 \%$-level. The moments concerning the inflation shock $u_{\Pi, t}$ also match the summary statistics of Table 1 well. In the model, the mean and $A R(1)$ coefficient of the inflation shock $u_{\Pi, t}$ are equal to zero by assumption (for any choice of parameters), as is the correlation between $u_{\Pi, t}$ and $\Delta c_{t}$. In the data, the values of these three moments are close to zero and not statistically significant at the $5 \%$-level. ${ }^{36}$

\footnotetext{
${ }^{33}$ We solve for all moments in closed form. Formulas are in Section 2 of the Online Appendix.

${ }^{34}$ For example, in a closely related model, Bansal and Shaliastovich $(2012)$ use $\psi=1.81$ and $\gamma=20.9$.

${ }^{35}$ For example, Bansal and Yaron (2004) use a standard deviation of $\Delta c_{t}$ of $2.93 \%$.

${ }^{36}$ The correlation between consumption and inflation is negative in monthly data but usually positive for samples that use
} 
From Panel A of Table 2, we match the coefficients $d_{0}^{u, 12}$ and $d_{1}^{u, 12}$ together with the low $R^{2}$, which guarantees that our model has the same low degree of unconditional predictability as the data. We also match the coefficients $d_{0}^{c, 12}$ and $d_{1}^{c, 12}$ and the higher $R^{2}$ of Panel B, which shows that the model has the same strong conditional predictability as the data. As mentioned before, we design our exogenous processes so that $d_{0}^{c, K}=0$ and $d_{1}^{c, K}=1$ for any calibration of the model and any $K$, consistent with the failure to reject the hypothesis that $d_{0}^{c, K}=0$ and $d_{1}^{c, K}=1$ in the data. In addition to the moments in Table 2, we match the volatility and $A R(1)$ coefficient of the nominal-real covariance, as measured by the first-stage coefficient $b_{t}^{12}=N R C_{t}^{C}$ from equation (3).

From Table 3, we match the first and second moments of returns and inflation betas for the two inflation portfolios with the highest and lowest inflation betas. Matching the returns of the High and Low portfolios ensures that the size of the inflation risk premium is consistent with the data. Matching the first moment of betas gives the appropriate mean quantity of inflation risk, while matching the second moment of betas gives the same degree of time-variation in the quantity of risk as in the data. Matching the mean returns and inflation betas of the High and Low portfolios automatically implies that we match the mean return and inflation beta of the High-minus-Low inflation beta-sorted portfolio, $I P_{t}^{H L}$, which for both returns and betas is simply their difference. Together, matching the inflation beta and returns for $I P_{t}^{H L}$ implies that the price of inflation risk in the model is consistent with the data. The model also matches two patterns from Fig. 3: the correlation between $\beta_{\Pi, H, t}$ and $\beta_{\Pi, L, t}$ is high ( 0.93 in the data and 1 in the model), and the volatility of the inflation beta of the $I P_{t}^{H L}$ portfolio is around one third of the volatility of the inflation betas of either the High or Low portfolio. From Table 5, we match the coefficient $L_{N R C}$ for the $I P_{t}^{H L}$ portfolio at the one-year horizon $(K=12)$, which shows that the model is capable of reproducing the empirical predictability in the price of inflation risk with the nominal-real covariance.

When we calibrate the aggregate market portfolio to have the same quantity of inflation risk as in the data, the model-implied aggregate stock market risk premium contains an inflation component that has the same magnitude and time-variation properties as the data. To calibrate the quantity of inflation risk of the market, we match the mean and standard deviation of the market's inflation beta, $\beta_{\Pi, m, t}$, as well as its correlation with $\beta_{\Pi, H, t}$ and $\beta_{\Pi, L, t}$. Once we have a calibrated $\beta_{\Pi, m, t}$, we use the $N R C_{t}^{C}$ implied by the model to run regression (9). We find that the key coefficient on the interaction term $\beta_{\Pi, m, t} \times N R C_{t}^{C}$ is 2.69 in the model, which is close to the empirical value of 2.85 in Table 7.

Last, the calibration we propose generates a timing premium for the early resolution of uncertainty that is considerably lower than in the long-run risk model, thus mitigating the criticism in Epstein, Farhi, and Strzalecki (2014). The timing premium is defined as the maximum share of consumption that the

quarterly or annual data. Our model allows for parameter combinations that give either sign for this correlation, although what matters most for asset pricing is the predictive relation between inflation and consumption rather than the contemporaneous correlation. 
representative agent is willing to forego every period to resolve all uncertainty at $t=1$. The first column in Table OA.8 of the Online Appendix shows that for our calibration the timing premium is $14.8 \%$, less than half the $31 \%$ timing premium in the baseline calibration of the long-run risk model. ${ }^{37}$ The last two columns of Table OA.8 show the low timing premium in our model arises because inflation is not as persistent as long-run risk, and not because of the persistence or volatility characteristics of the nominal-real covariance process $\varphi_{t}$.

\section{Robustness checks}

We discuss a range of robustness checks for which we report results in Section 3 of the Online Appendix.

\section{Benchmark Asset Pricing Factors and Predictors}

We test whether the inflation risk premium and its variation with the nominal-real covariance are robust to controlling for the factors in the CAPM, Fama-French three- and five-factor models (FF3M, FF5M), and the Carhart four-factor model (FFCM). First, we include these factors as control variables when estimating stocks' ex ante inflation exposures using Eq. (4). Table OA.10 presents the predictive regressions for these alternative estimates of the inflation risk premium. We see that both the unconditional inflation risk premium (as measured by the intercept of the predictive regression) as well as the coefficient on the nominal-real covariance are robust, both in magnitude and significance.

Next, we control for these benchmark factors ex post in the predictive regression in Eq. (7). Table OA.11 shows that the unconditionally negative inflation risk premium is captured in the FFCM and FF5M. In contrast, we find that the coefficient on the nominal-real covariance remains economically large and at least marginally significant in all models. We thus conclude that time-variation in the inflation risk premium is not due to exposure to benchmark factors, neither ex ante nor ex post.

Table OA.12 runs the predictive regressions of Eq. (7) controlling for standard Intertemporal CAPM predictors: dividend yield (DY), default spread (DS), and term spread (TS) as well as the consumption-wealth ratio (CAY). We find that the inflation risk premium varies significantly with the nominal-real covariance even after controlling for these benchmark predictors and conclude that the nominal-real covariance represents a new source of conditioning information.

Alternative Measures of Inflation and Sorting Procedures

Table OA.13 analyzes alternative measures of inflation risk. We consider raw inflation; an AR(1)innovation in inflation; the monthly change in annual inflation; and, real-time vintage CPI inflation. The latter test represents a truly out-of-sample exercise, as we skip a month after portfolio formation to take

\footnotetext{
${ }^{37}$ For comparison, an i.i.d. model has a premium of $10 \%$, the long-run risk model without stochastic volatility has a premium of $29 \%$, and the long run-risk model with stochastic volatility and preference parameters equal to the ones we use $(\gamma=15$ and $\psi=2$ ) has a premium of $44 \%$ (Table OA.9 in the Online Appendix).
} 
into account the reporting delay in inflation data. Focusing on predictability of the High-minus-Low inflation beta-sorted portfolio return, we find that our benchmark ARMA $(1,1)$ specification represents a lower bound. Two alternative measures provide particularly strong evidence for time-variation in the inflation risk premium with the nominal-real covariance. Using raw inflation or real-time inflation, the $R^{2}$ more than doubles to about $15 \%$ with a coefficient estimate that is over $7 \%$ and a $t$-statistic over three. Moreover, the unconditional inflation risk premium is negative in all cases.

Table OA.14 shows that our results are also robust to changes in the method of estimating inflation betas. We again focus on High-minus-Low portfolios that are constructed by sorting stocks on inflation betas estimated using (i) weighted least squares plus shrinkage (WLS) or ordinary least squares (OLS); (ii) a sort where each portfolio comes from a double sort on inflation beta and size (Size) or a single sort on beta (No Size), and (iii) ARMA(1,1) innovations in inflation (ARMA) or raw inflation (Inflation). In all cases, the coefficient on the nominal-real covariance is positive, economically large, and at least marginally significant. In three out of eight cases, the $t$-statistic on the nominal-real covariance is well above three. Finally, Table OA.15 replicates our main analysis using quarterly data. We find that the High-minus-Low inflation risk premium (estimated by sorting stocks on exposure in quarterly returns to ARMA(1,1)-innovations in quarterly inflation) is similarly predictably by the nominal-real covariance (estimated as the rolling coefficient in a regression of quarterly consumption growth on lagged quarterly inflation).

\section{Time-Varying Inflation Betas}

In the model of Section 6., inflation betas are linear in the nominal-real covariance. To check if our results are robust to this specification of inflation betas, we adjust Eq. (4) as follows:

$$
\left(\widehat{\alpha_{p, t}}, \widehat{\beta_{\Pi, p, t}}\right)=\underset{\alpha_{p, t}, \beta_{\Pi, p, t}^{0}, \beta_{\Pi, p, t}^{1}}{\arg \min } \sum_{\tau=1}^{t} K(\tau)\left(R_{i, \tau}-\alpha_{p, t}-\left(\beta_{\Pi, p, t}^{0}+\beta_{\Pi, p, t}^{1} N R C_{t-1}^{C}\right) u_{\Pi, \tau}\right)^{2} .
$$

We find that the linear conditional inflation betas for each decile portfolio $p, \beta_{\Pi, p, t}=\beta_{\Pi, p, t}^{0}+\beta_{\Pi, p, t}^{1} N R C_{t-1}^{C}$, are highly correlated to the betas we use in Section 5.1. (at a correlation of 0.79 on average). Moreover, Table OA.16 shows that the results from our pooled regressions are, if anything, stronger than what we report already in Table 6 . Both unconditional variation in inflation betas across portfolios as well as time-variation in the average inflation beta interact with the nominal-real covariance to generate large and significant timevariation in the returns of inflation beta-sorted portfolios.

\section{Monetary Policy (Risk)}

We perform two tests to assess the impact of monetary policy on our results. First, Campbell, Pflueger, and Viceira (2015) link time-variation in the nominal-real covariance to three periods: the period of rising inflation up to the first quarter of 1977, the inflation-fighting period under Volcker and Greenspan up to the last quarter of 2000, and the recent period of increased central bank transparency, gradualism, and renewed attention to output stabilization. Table OA.17 shows that our conclusions on the interaction between inflation 
beta and the nominal-real covariance are robust to including in the pooled regression a dummy for each of these subperiods. This finding highlights that our empirical evidence on the inflation risk premium is richer than a sample split into long-lasting monetary policy regimes.

Next, we analyze whether monetary policy risk can drive our results. Table OA.18 presents the exposures of our inflation-beta sorted portfolios to Romer and Romer (2004) monetary policy shocks and changes in the federal funds rate (also used in, e.g., Neuhierl and Weber, 2017; Velikov, 2017). Whereas exposure to changes in the federal funds rate is typically negative and significant, there is no clear pattern from High to Low inflation beta, neither in the full sample nor within any of the three monetary policy regimes. As a result, exposures for the High-minus-Low portfolio $\left(I P_{t}^{H L}\right)$ are small and insignificant. Similar results obtain for the Romer and Romer (2004) shocks. We conclude that monetary policy risk is unlikely to explain the variation in the inflation risk premium that we uncover. This conclusion is consistent with Velikov (2017), who argues that exposure to monetary policy shocks is transient and best observed in high frequency stock returns. In contrast, our exposures to inflation risk are persistent.

\section{Bond Returns}

Although our focus is on the inflation risk premium in the stock market, we can examine whether similar results apply in the bond market. We use returns on constant maturity Treasury bonds (with maturities from one to thirty years) in excess of the one-month T-bill return. In Panel A of Table OA.19, we see that average excess returns are increasing in maturity, whereas inflation beta is decreasing in maturity. The ten-minus-one-year maturity difference in average excess return is $1.44 \%$. Dividing this number by the ten-minus-one-year difference in inflation beta gives an estimate of the unconditional price of inflation risk: $1.44 /(-1.16)=-1.24 \%$. This price is close to what we estimate in the stock market in Table 3 : $-4.23 / 3.00=-1.41 \%$.

Having said that, the evidence in Panel B shows that the nominal-real covariance is not a strong predictor of excess bond returns in the time-series. We report coefficient estimates from a regression of annual excess bond returns on the lagged nominal-real covariance. Depending on maturity, a one standard deviation increase in the nominal-real covariance predicts an increase in excess bond return between $-0.81 \%$ and $0.41 \%$. Although non-negligible economically, the estimated coefficient is only statistically significant at the 1\%-level for the one-year maturity bond and at the 10\%-level for the two-year maturity bond.

\section{Conclusion}

We show that inflation risk is priced in the stock market in a time-varying way. We study a key driver of this time-variation: the nominal-real covariance, which is the time-varying relation between inflation and real future consumption growth.

Our main empirical finding is that the inflation risk premium increases by a large and significant $5.3 \%$ for a standard deviation increase in the nominal-real covariance. Given an unconditional inflation risk premium 
of $-4.2 \%$, this result implies that the inflation risk premium can change sign. The economic intuition is that when inflation predicts consumption growth with a negative sign as it did for example in the 1970s, stocks with a high inflation beta are attractive as a hedge against bad states of nature and thus command relatively low expected returns. When inflation predicts consumption growth with a positive sign, as it did since the early 2000s, high inflation beta stocks are risky (as they covary negatively with marginal utility) and the inflation risk premium switches sign.

By decomposing the time variation in the inflation risk premium into variation in the price and quantity of risk, we obtain a rich set of results that are new to the literature. The time-variation in the inflation risk premium between high and low inflation beta stocks is driven by time-variation in the price of inflation risk. Quantities do matter for the average inflation risk premium across stocks, however. The reason is that time-variation in the quantity of inflation risk is largely common across stocks. Consequently, the market risk premium contains an inflation component that is time-varying due to both the price and quantity of risk.

We develop an equilibrium model that builds on the empirical observation that inflation today predicts real consumption growth in the future in a time-varying way. In the model, this time-variation is controlled by the nominal-real covariance, which allows us to closely match the observed dynamics in the price and quantities of inflation risk in both the cross-section of stocks and the aggregate market. Our theoretical

contribution is to extend the long-run risk framework by using an observable state variable that is related to future consumption growth in a time-varying way.

\section{References}

Ang, A., Bekaert, G., Wei, M., 2007. Do macro variables, asset markets, or surveys forecast inflation better? Journal of Monetary Economics 54, 1163-1212.

Ang, A., Bekaert, G., Wei, M., 2008. The term structure of real rates and expected inflation. Journal of Finance 63, 797-849.

Ang, A., Brière, M., Signori, O., 2012. Inflation and individual equities. Financial Analysts Journal 68, 36-55.

Ang, A., Piazzesi, M., 2003. A no-arbitrage vector autoregression of term structure dynamics with macroeconomic and latent variables. Journal of Monetary economics 50, 745-787.

Ang, A., Piazzesi, M., Wei, M., 2006. What does the yield curve tell us about GDP growth? Journal of econometrics 131, 359-403.

Bansal, R., Kiku, D., Yaron, A., 2012. An empirical evaluation of the long-run risks model for asset prices. Critical Finance Review 1, 183-221.

Bansal, R., Shaliastovich, I., 2012. A long-run risks explanation of predictability puzzles in bond and currency markets. Review of Financial Studies 26, 1-33. 
Bansal, R., Yaron, A., 2004. Risks for the long run: A potential resolution of asset pricing puzzles. Journal of Finance 59, 1481-1509.

Bekaert, G., Wang, X., 2010. Inflation risk and the inflation risk premium. Economic Policy 25, 755-806.

Bilal, M. A., 2017. Zeroing in: Asset pricing at the zero lower bound. Unpublished working paper. New York University.

Boons, M., 2016. State variables, macroeconomic activity, and the cross section of individual stocks. Journal of Financial Economics 119, 489-511.

Boudoukh, J., Richardson, M., Whitelaw, R. F., 1994. Industry returns and the Fisher effect. Journal of Finance 49, 1595-1615.

Breeden, D. T., Gibbons, M. R., Litzenberger, R. H., 1989. Empirical tests of the consumption-oriented CAPM. Journal of Finance 44, 231-262.

Bretscher, L., Hsu, A., Tamoni, A., 2017. Level and volatility shocks to fiscal policy: Term structure implications. Unpublished working paper. London Business School.

Campbell, J. Y., 1991. A variance decomposition for stock return. The Economic Journal 101, 157-179.

Campbell, J. Y., Ammer, J., 1993. What moves the stock and bond markets? a variance decomposition for long-term asset returns. The Journal of Finance 48, 3-37.

Campbell, J. Y., Giglio, S., Polk, C., Turley, R., 2018. An intertemporal capm with stochastic volatility. Journal of Financial Economics 128, 207-233.

Campbell, J. Y., Pflueger, C., Viceira, L. M., 2015. Monetary policy drivers of bond and equity risks. Unpublished working paper. Harvard University.

Campbell, J. Y., Sunderam, A., Viceira, L. M., 2017. Inflation bets or deflation hedges? The changing risks of nominal bonds. Critical Finance Review 6, 263-301.

Campbell, J. Y., Viceira, L. M., 2002. Strategic Asset Allocation: Portfolio Choice for Long-Term Investors. Oxford University Press, New York.

Campbell, J. Y., Vuolteenaho, T., 2004. Bad beta, good beta. American Economic Review 94, 1249-1275.

Carhart, M. M., 1997. On persistence in mutual fund performance. Journal of Finance 52, 57-82.

Chen, H., Dou, W. W., Kogan, L., 2015. Measuring the dark matter in asset pricing models. Unpublished working paper. Massachussetts Institute of Technology. 
Chen, N.-F., Roll, R., Ross, S. A., 1986. Economic forces and the stock market. Journal of Business 59, $383-403$.

Chen, R.-R., Liu, B., Cheng, X., 2010. Pricing the term structure of inflation risk premia: Theory and evidence from TIPS. Journal of Empirical Finance 17, 702-721.

Chordia, T., Goyal, A., Shanken, J. A., 2015. Cross-sectional asset pricing with individual stocks: betas versus characteristics. Unpublished working paper. Emory University, University of Lusanne.

Cochrane, J. H., 2005. Asset Pricing, vol. 1. Princeton University Press, Princeton, NJ.

Cochrane, J. H., 2017. Macro-finance. Review of Finance 21, 945-985.

Cosemans, M., Frehen, R., Schotman, P. C., Bauer, R., 2016. Estimating security betas using prior information based on firm fundamentals. Review of Financial Studies 29, 1072-1112.

Dai, Q., Singleton, K. J., Yang, W., 2007. Regime shifts in a dynamic term structure model of US treasury bond yields. Review of Financial Studies 20, 1669-1706.

David, A., Veronesi, P., 2013. What ties return volatilities to price valuations and fundamentals? Journal of Political Economy 121, 682-746.

Driscoll, J. C., Kraay, A. C., 1998. Consistent covariance matrix estimation with spatially dependent panel data. Review of Economics and Statistics 80, 549-560.

Duffee, G., 2018. Expected inflation, real rates, and stock-bond comovement. Unpublished working paper. Johns Hopkins University.

Elton, E. J., Gruber, M. J., Urich, T. J., 1978. Are betas best? Journal of Finance 33, 1375-1384.

Engsted, T., Pedersen, T. Q., Tanggaard, C., 2012. Pitfalls in var based return decompositions: A clarification. Journal of Banking \& Finance 36, 1255-1265.

Epstein, L. G., Farhi, E., Strzalecki, T., 2014. How much would you pay to resolve long-run risk? American Economic Review 104, 2680-2697.

Epstein, L. G., Zin, S. E., 1989. Substitution, risk aversion, and the temporal behavior of consumption and asset returns: A theoretical framework. Econometrica: Journal of the Econometric Society 57, 937-969.

Fama, E. F., 1976. Foundations of Finance: Portfolio Decisions and Securities Prices. Basic Books (AZ).

Fama, E. F., French, K. R., 1993. Common risk factors in the returns on stocks and bonds. Journal of Fnancial Economics 33, 3-56. 
Fama, E. F., French, K. R., 2008. Dissecting anomalies. Journal of Finance 63, 1653-1678.

Fama, E. F., French, K. R., 2015. A five-factor asset pricing model. Journal of Financial Economics 116, $1-22$.

Fama, E. F., Gibbons, M. R., 1984. A comparison of inflation forecasts. Journal of Monetary Economics 13, $327-348$.

Fama, E. F., MacBeth, J. D., 1973. Risk, return, and equilibrium: Empirical tests. Journal of Political Economy 81, 607-636.

Ferson, W. E., Harvey, C. R., 1991. The variation of economic risk premiums. Journal of Political Economy $99,385-415$.

Fuhrer, J., Moore, G., 1995. Inflation persistence. Quarterly Journal of Economics 110, 127-159.

Gabaix, X., 2008. Variable rare disasters: A tractable theory of ten puzzles in macro-finance. American Economic Review 98, 64-67.

Gorodnichenko, Y., Weber, M., 2016. Are sticky prices costly? evidence from the stock market. American Economic Review 106, 165-99.

Gourio, F., Ngo, P., 2016. Risk premia at the ZLB: a macroeconomic interpretation. Unpublished working paper. Federal Reserve Bank of Chicago.

Gürkaynak, R. S., Sack, B., Wright, J. H., 2010. The TIPS yield curve and inflation compensation. American Economic Journal: Macroeconomics 2, 70-92.

Harvey, C. R., Liu, Y., Zhu, H., 2016. ... and the cross-section of expected returns. Review of Financial Studies 29, 5-68.

Haubrich, J., Pennacchi, G., Ritchken, P., 2012. Inflation expectations, real rates, and risk premia: Evidence from inflation swaps. Review of Financial Studies 25, 1588-1629.

Kan, R., Zhang, C., 1999. Two-pass tests of asset pricing models with useless factors. Journal of Finance 54, 203-235.

Kang, J., Pflueger, C. E., 2015. Inflation risk in corporate bonds. Journal of Finance 70, 115-162.

Koijen, R. S., Lustig, H., Van Nieuwerburgh, S., 2017. The cross-section and time series of stock and bond returns. Journal of Monetary Economics 88, 50-69.

Kreps, D. M., Porteus, E. L., 1978. Temporal resolution of uncertainty and dynamic choice theory. Econometrica: journal of the Econometric Society 46, 185-200. 
Le, A., Singleton, K. J., 2010. An equilibrium term structure model with recursive preferences. American Economic Review 100, 557-561.

Le, A., Singleton, K. J., Dai, Q., 2010. Discrete-time affine q term structure models with generalized market prices of risk. Review of Financial Studies 23, 2184-2227.

Lettau, M., Ludvigson, S., 2001a. Consumption, aggregate wealth, and expected stock returns. Journal of Finance 56, 815-849.

Lettau, M., Ludvigson, S., 2001b. Resurrecting the (C) CAPM: A cross-sectional test when risk premia are time-varying. Journal of Political Economy 109, 1238-1287.

Levi, Y., Welch, I., 2017. Best practice for cost-of-capital estimates. Journal of Financial and Quantitative Analysis 52, 427-463.

Lintner, J., 1965. The valuation of risk assets and the selection of risky investments in stock portfolios and capital budgets. Review of Economics and Statistics 47, 13-37.

Maio, P., Santa-Clara, P., 2012. Multifactor models and their consistency with the ICAPM. Journal of Financial Economics 106, 586-613.

Modigliani, F., Cohn, R. A., 1979. Inflation, rational valuation and the market. Financial Analysts Journal $35,24-44$.

Mossin, J., 1966. Equilibrium in a capital asset market. Econometrica: Journal of the Econometric Society $34,768-783$.

Neuhierl, A., Weber, M., 2017. Monetary policy slope and the stock market. Unpublished working paper. University of Chicago Booth School of Business.

Patton, A. J., Timmermann, A., 2010. Monotonicity in asset returns: New tests with applications to the term structure, the CAPM, and portfolio sorts. Journal of Financial Economics 98, 605-625.

Petkova, R., 2006. Do the Fama-French factors proxy for innovations in predictive variables? Journal of Finance 61, 581-612.

Piazzesi, M., Schneider, M., 2006. Equilibrium yield curves. NBER macroeconomics Annual 21, 389-472.

Romer, C. D., Romer, D. H., 2004. A new measure of monetary shocks: Derivation and implications. American Economic Review 94, 1055-1084.

Sharpe, W. F., 1964. Capital asset prices: A theory of market equilibrium under conditions of risk. Journal of Finance 19, 425-442. 
Song, D., 2017. Bond market exposures to macroeconomic and monetary policy risks. Review of Financial Studies 30, 2761-2817.

Stock, J. H., Watson, M. W., 2005. Implications of dynamic factor models for VAR analysis. National Bureau of Economic Research Working Paper 11467.

Vasicek, O. A., 1973. A note on using cross-sectional information in bayesian estimation of security betas. Journal of Finance 28, 1233-1239.

Vassalou, M., 2000. Exchange rate and foreign inflation risk premiums in global equity returns. Journal of International Money and Finance 19, 433-470.

Vassalou, M., 2003. News related to future GDP growth as a risk factor in equity returns. Journal of Financial Economics 68, 47-73.

Velikov, M., 2017. FOMC announcements and predictable returns. Unpublished working paper. Federal Reserve Bank of Richmond.

Wachter, J. A., 2006. A consumption-based model of the term structure of interest rates. Journal of Financial Economics 79, 365-399.

Weber, M., 2015. Nominal rigidities and asset pricing. Unpublished working paper. University of Chicago Booth School of Business.

Welch, I., Goyal, A., 2007. A comprehensive look at the empirical performance of equity premium prediction. Review of Financial Studies 21, 1455-1508. 


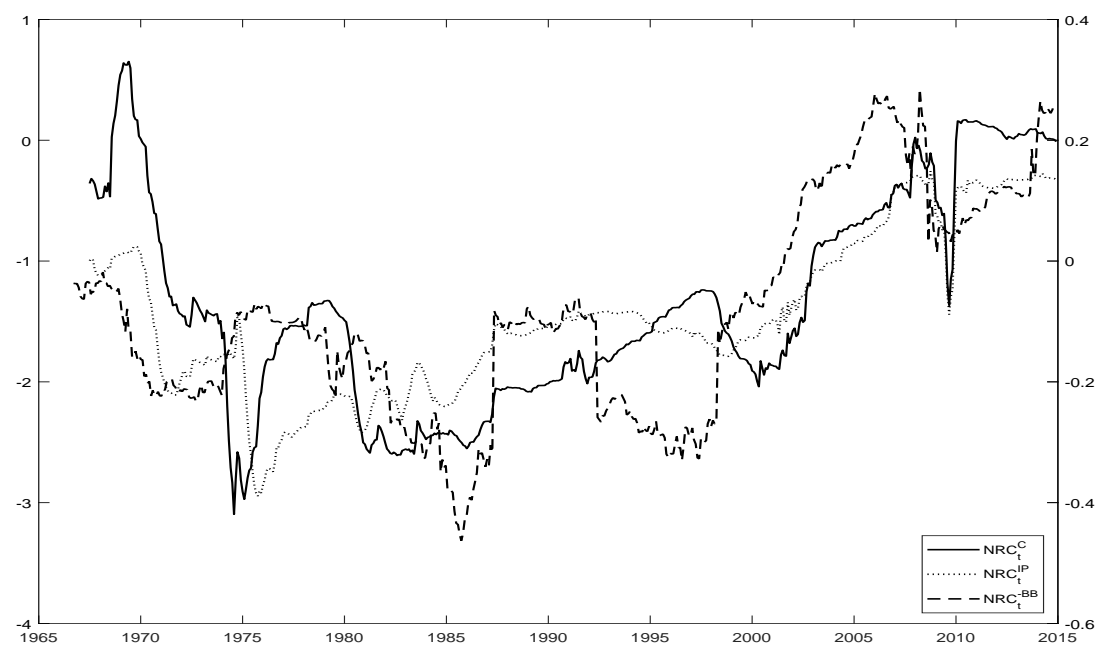

Figure 1: Alternative Measures of the Nominal-Real Covariance

We present three measures of the nominal-real covariance. The first is the coefficient in a rolling regression of twelvemonth consumption growth on lagged inflation $\left(N R C_{t}^{C}\right)$. The second measure substitutes industrial production for consumption $\left(N R C_{t}^{I P}\right)$. These measures are plotted on the left axis and $N R C_{t}^{I P}$ is divided by four to preserve scaling. The third measure is the negative of the stock market beta of the 10-year constant maturity treasury bond $\left(N R C_{t}^{-B B}\right.$, right axis).

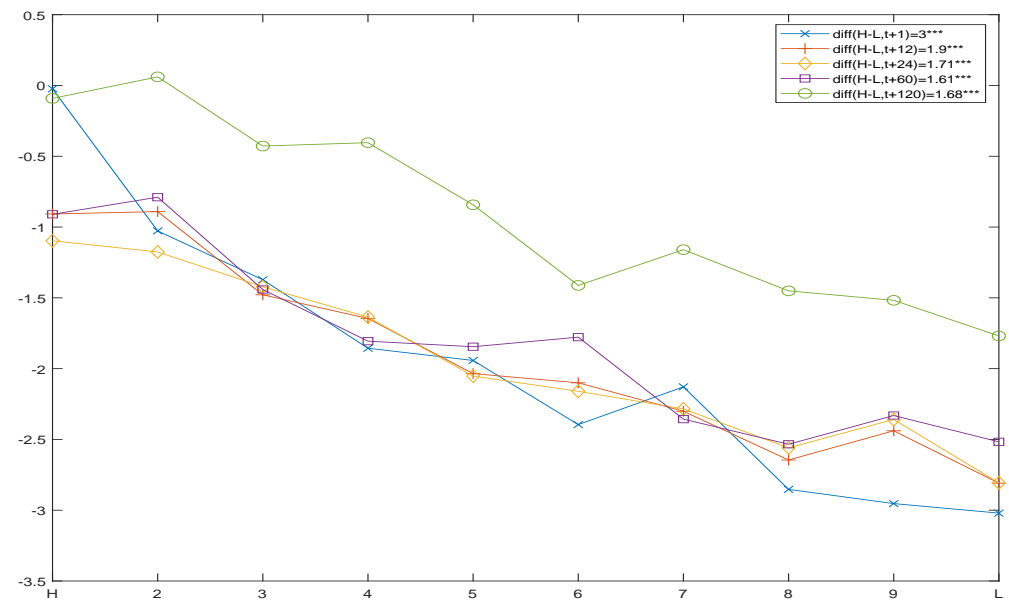

Figure 2: Post-Ranking Inflation Beta After Sorting

We plot the monthly post-ranking inflation beta for the High-minus-Low portfolio one month, one year, two years, five years, and ten years after sorting. The legend includes the estimated inflation beta and $*, * *, * * *$ indicate statistical significance at the 10, 5, and 1\%-level using Newey-West(1) standard errors. 


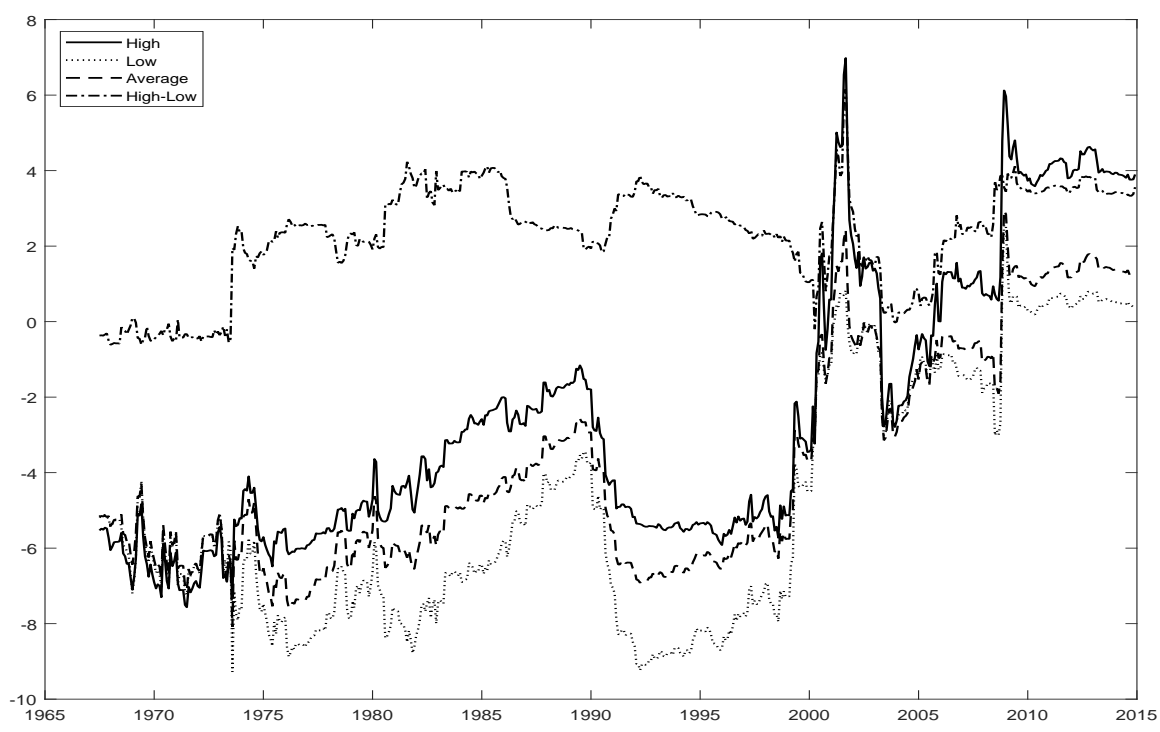

Figure 3: Post-Ranking Inflation Betas over Time

We present the inflation beta of the High, Low, and High-minus-Low inflation beta decile portfolio as well as the average inflation beta over the ten portfolios. 
Table 1: Descriptive Statistics

This table presents descriptive statistics in annualized percentages for CPI inflation $\left(\Pi_{t}\right)$, ARMA $(1,1)$-innovations in inflation $\left(u_{\Pi, t}\right)$, consumption growth $\left(\Delta C_{t}\right)$, the aggregate stock market excess return $\left(R_{m, t}\right)$, and the one month T-bill return $\left(R_{f, t}\right)$. $\operatorname{AR}(1)$ is the first-order autocorrelation coefficient. The sample period is from July 1962 to December 2014.

\begin{tabular}{lccccc}
\hline & $\Pi_{t}$ & $u_{\Pi, t}$ & $\Delta C_{t}$ & $R_{m, t}$ & $R_{f, t}$ \\
& & & & & \\
\hline Mean & 3.93 & 0.11 & 1.97 & 6.45 & 4.85 \\
St. dev. & 1.11 & 0.85 & 1.13 & 15.44 & 0.89 \\
AR(1) & 62.47 & 15.35 & -16.84 & 7.47 & 96.90 \\
& & & & & \\
& & Correlations & & \\
$\Pi_{t}$ & 1 & 79.03 & -17.75 & -13.35 & 50.35 \\
$u_{\Pi, t}$ & & 1 & -17.41 & -11.72 & 15.35 \\
$\Delta C_{t}$ & & & 1 & 17.45 & 1.26 \\
$R_{m, t}$ & & & & 1 & -8.68 \\
$R_{f, t}$ & & & & & 1 \\
\hline
\end{tabular}


Table 2: Unconditional and Conditional Predictive Regressions of Consumption Growth on Inflation

In this table we present predictive regressions of consumption growth, $\Delta C_{t+1: t+K}$ over horizons $K=1,3,6,12$, on lagged inflation, $\Pi_{t}$, from July 1967 to December 2014. Panel A presents an unconditional regression: $\Delta C_{t+1: t+K}=d_{0}^{u, K}+d_{1}^{u, K} \Pi_{t}+$ $e_{t+1: t+K}$. Panel $\mathrm{B}$ presents a two-stage conditional regression. In the first stage, we regress consumption growth on lagged inflation over a backward looking rolling window: $\Delta C_{s+1: s+K}=a_{t}^{K}+b_{t}^{K} \Pi_{s}+e_{s+1: s+K}$, for $s=1, \ldots, t-K$. Combining the estimated coefficients with inflation in month $t$, we then predict future consumption growth in the full time series: $\Delta C_{t+1: t+K}=$ $d_{0}^{c, K}+d_{1}^{c, K}\left(\widehat{a_{t}^{K}}+\widehat{b_{t}^{K}} \Pi_{t}\right)+e_{t+1: t+K}$. For this conditional setup, we present also an out-of-sample $R^{2}$, which compares the performance of the conditional model that includes inflation with a conditional model that includes only a constant. To be precise, $R^{2}-O O S=1-\frac{\operatorname{Var}\left(\Delta C_{t+1: t+K}-\widehat{\left.\left(a_{t}^{K}+\widehat{b_{t}^{K}} \Pi_{t}\right)\right)}\right.}{\operatorname{Var}\left(\Delta C_{t+1: t+K}-\widehat{a_{t}^{*}, K}\right)}$, where $\widehat{a_{t}^{*, K}}$ is estimated through a backward-looking regressing of consumption growth on a constant at each point in time (thus setting $b_{t}^{K}=0$ in the first stage). In both panels, we report $t$-statistics using Newey-West standard errors with $K$ lags. In Panel B, we also report block-bootstrapped $t$-statistics using standard errors calculated as the standard deviation of coefficient estimates in 1000 bootstrap replications. We report these $t$-statistics for two null hypotheses: $H_{0}: d_{1}^{c, K}=0$ and $H_{0}: d_{1}^{c, K}=1 . R^{2}$ 's are reported in percentage points.

\begin{tabular}{|c|c|c|c|c|c|c|c|c|c|}
\hline \multicolumn{5}{|c|}{ Panel A: Unconditional } & \multicolumn{5}{|c|}{ Panel B: Conditional } \\
\hline Horizon $K$ & 1 & 3 & 6 & 12 & & 1 & 3 & 6 & 12 \\
\hline$d_{0}^{u, K}$ & 0.00 & 0.01 & 0.01 & 0.02 & $d_{0}^{c, K}$ & 0.00 & 0.00 & 0.00 & 0.00 \\
\hline \multirow[t]{2}{*}{$t$} & $(9.10)$ & $(8.73)$ & $(7.78)$ & $(7.52)$ & $t$ & $(0.76)$ & $(0.15)$ & $(0.07)$ & $(0.10)$ \\
\hline & & & & & $t_{\text {boot }}$ & $(0.42)$ & $(0.09)$ & $(0.04)$ & $(0.05)$ \\
\hline$d_{1}^{u, K}$ & -0.07 & -0.15 & -0.20 & -0.50 & $d_{1}^{c, K}$ & 0.77 & 0.84 & 0.84 & 0.81 \\
\hline \multirow[t]{4}{*}{$t\left(d_{1}^{u, K}=0\right)$} & $(-1.37)$ & $(-1.08)$ & $(-0.77)$ & $(-1.06)$ & $t\left(d_{1}^{c, K}=0\right)$ & $(4.74)$ & $(5.60)$ & $(5.30)$ & $(4.56)$ \\
\hline & & & & & $t_{\text {boot }}\left(d_{1}^{c, K}=0\right)$ & $(2.57)$ & $(2.74)$ & $(2.24)$ & $(1.77)$ \\
\hline & & & & & $t\left(d_{1}^{c, K}=1\right)$ & $(-1.44)$ & $(-1.04)$ & $(-1.01)$ & $(-1.06)$ \\
\hline & & & & & $t_{\text {boot }}\left(d_{1}^{c, K}=1\right)$ & $(-0.78)$ & $(-0.51)$ & $(-0.43)$ & $(-0.41)$ \\
\hline$R^{2}$ & 0.26 & 0.76 & 0.44 & 1.12 & $R^{2}$ & 3.29 & 10.29 & 13.28 & 14.92 \\
\hline & & & & & $R^{2}-O O S$ & 1.80 & 4.60 & 6.70 & 10.41 \\
\hline
\end{tabular}




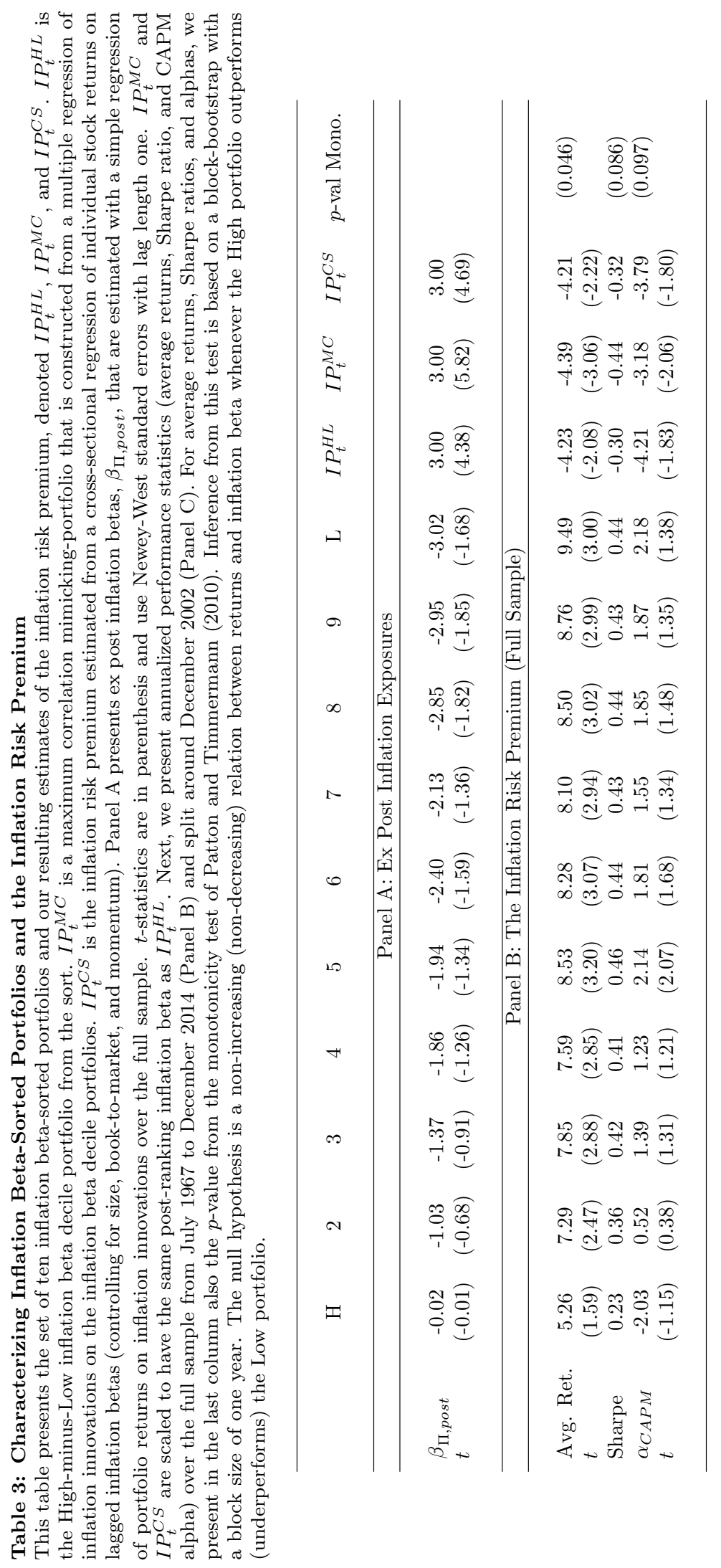




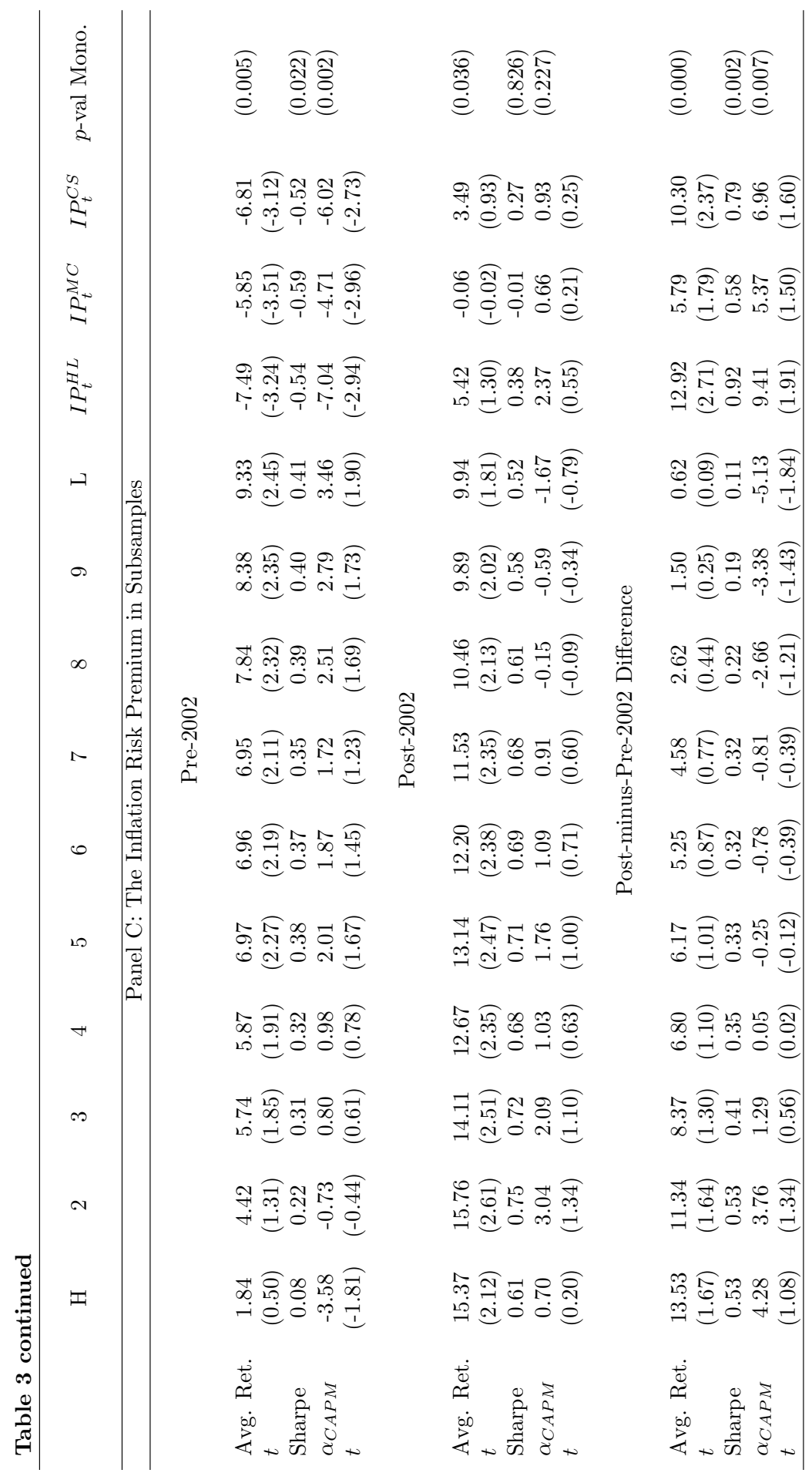


Table 4: Exposures to Breakeven Inflation

This table presents the exposure of inflation beta-sorted portfolios to first-differences in breakeven inflation (denoted $\Delta B E_{t}$ ). Breakeven inflation is the difference between the yield on a ten year constant maturity nominal Treasury bond and its TIPS equivalent. The exposures are estimated with the OLS time-series regression $R_{p, t}=a_{p}+\beta_{B E, p} \Delta B E_{t}+e_{p, t}$. In Panel $\mathrm{A}$, we use the returns of portfolios sorted on exposure to $\operatorname{ARMA}(1,1)$-innovations in inflation $\left(u_{\Pi, t}\right)$, as in Table 3 . In Panel B, we use the returns of decile portfolios sorted on exposure to $\Delta B E_{t}$. The exposure of each stock to $\triangle B E$ is estimated with an OLS regression over a 60 month rolling window. We report the coefficients $\beta_{B E, p}$ (with $t$-statistics in parentheses) and the $R^{2}$ for each of the regressions. The sample for both panels is January 2003 (when TIPS data becomes available) to December 2014.

\begin{tabular}{|c|c|c|c|c|c|c|c|c|c|c|c|}
\hline & High & 2 & 3 & 4 & 5 & 6 & 7 & 8 & 9 & Low & $I P_{t}^{H L}$ \\
\hline \multicolumn{12}{|c|}{ Panel A: Portfolios sorted on exposure to ARMA(1,1)-innovations in inflation $\left(u_{\Pi, t}\right)$} \\
\hline$\beta_{B E, p}$ & $\begin{array}{l}17.84 \\
(2.25)\end{array}$ & $\begin{array}{l}11.43 \\
(1.73)\end{array}$ & $\begin{array}{c}9.36 \\
(1.46)\end{array}$ & $\begin{array}{c}9.31 \\
(1.49)\end{array}$ & $\begin{array}{c}9.68 \\
(1.66)\end{array}$ & $\begin{array}{c}8.52 \\
(1.45)\end{array}$ & $\begin{array}{c}8.87 \\
(1.49)\end{array}$ & $\begin{array}{c}8.09 \\
(1.42)\end{array}$ & $\begin{array}{c}6.98 \\
(1.08)\end{array}$ & $\begin{array}{c}8.05 \\
(1.13)\end{array}$ & $\begin{array}{c}9.79 \\
(4.12)\end{array}$ \\
\hline$R^{2}$ & 13.09 & 7.46 & 5.60 & 6.07 & 6.85 & 5.61 & 6.76 & 5.46 & 3.95 & 4.20 & 11.87 \\
\hline \multicolumn{12}{|c|}{ Panel B: Portfolios sorted on exposure to changes in breakeven inflation $\left(\Delta B E_{t}\right)$} \\
\hline$\beta_{B E, p}$ & $\begin{array}{l}21.09 \\
(2.22)\end{array}$ & $\begin{array}{l}16.88 \\
(1.93)\end{array}$ & $\begin{array}{l}15.38 \\
(1.98)\end{array}$ & $\begin{array}{l}12.82 \\
(1.54)\end{array}$ & $\begin{array}{c}12.86 \\
(1.63)\end{array}$ & $\begin{array}{l}10.53 \\
(1.60)\end{array}$ & $\begin{array}{c}9.27 \\
(1.38)\end{array}$ & $\begin{array}{c}7.29 \\
(1.09)\end{array}$ & $\begin{array}{c}8.19 \\
(1.31)\end{array}$ & $\begin{array}{c}8.61 \\
(1.03)\end{array}$ & $\begin{array}{l}12.47 \\
(4.82)\end{array}$ \\
\hline$R^{2}$ & 16.40 & 13.66 & 13.35 & 10.43 & 11.21 & 9.88 & 7.58 & 5.28 & 7.09 & 5.08 & 20.01 \\
\hline
\end{tabular}




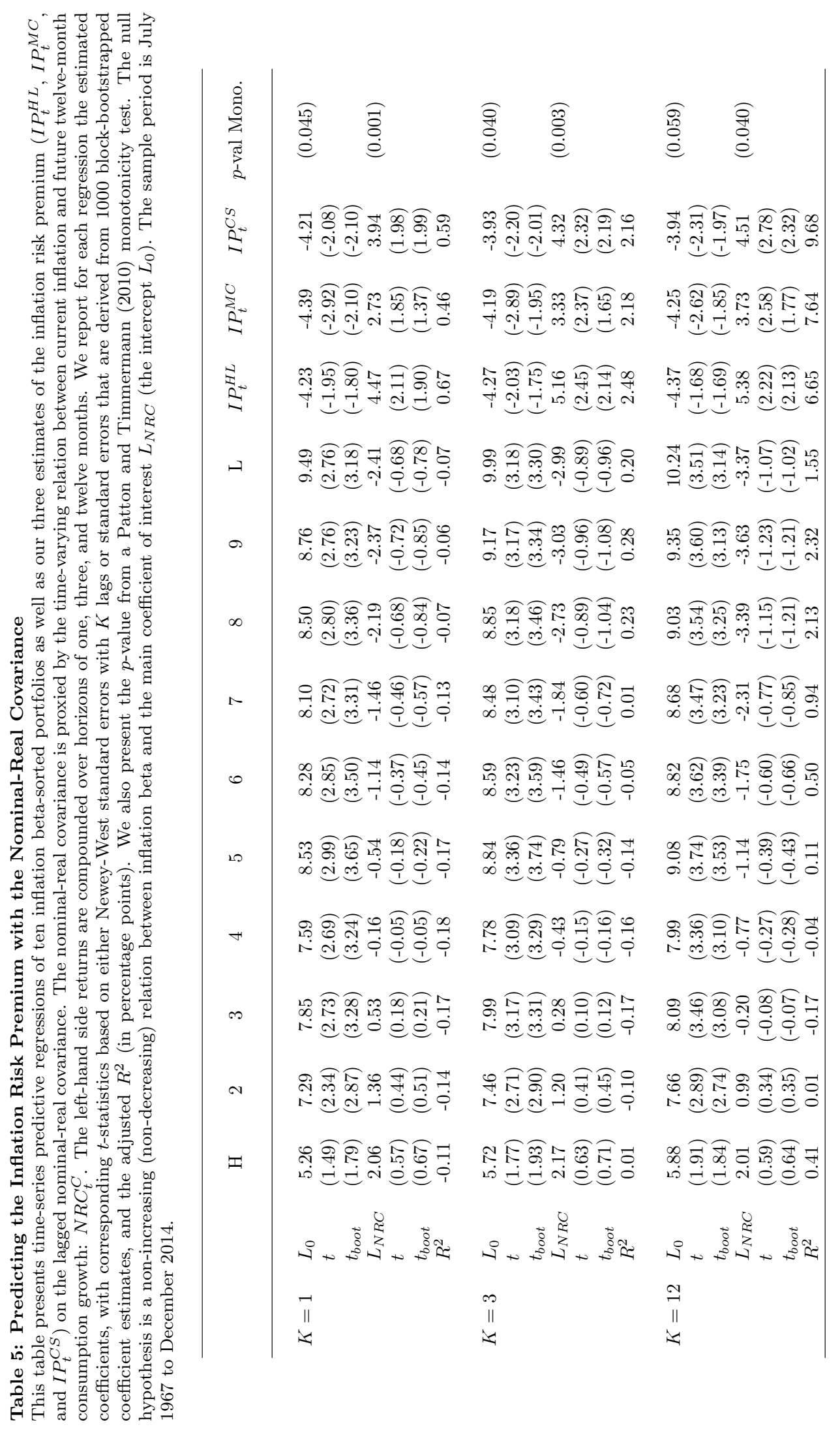


Table 6: Time-Varying Inflation Risk Premia in Pooled Regressions

Panel A of this table presents the estimated coefficients (with asymptotic Driscoll and Kraay (1998) standard errors in parentheses) from pooled predictive regressions of annual returns of the inflation beta-sorted decile portfolios on their lagged inflation beta $\left(\beta_{\Pi, p, t}\right)$, the nominal-real covariance $\left(N R C_{t}^{C}\right)$, and an interaction term: $R_{p, t+1: t+12}=L_{0}+L_{\beta_{\Pi}} \beta_{\Pi, p, t}+L_{N R C} N R C_{t}^{C}+$ $L_{\beta_{\Pi} \times N R C}\left(\beta_{\Pi, p, t} \times N R C_{t}^{C}\right)+\varepsilon_{p, t+1: t+12}$. In Model [1], we set $L_{\beta_{\Pi} \times N R C}=0$, whereas Model [2] estimates all three coefficients freely. For the latter model, Panel B presents the predicted risk premia (with asymptotic and bootstrapped standard errors in parentheses) in four distinct cases. That is, when $\beta_{\Pi, p, t}$ and $N R C_{t}^{C}$ are at plus or minus one standard deviation from their respective means in the pool. Models [3] to [5] in Panel A analyze which components of inflation betas interact with the nominal-real covariance. Model [3] replaces the interaction term, $\beta_{\Pi, p, t} \times N R C_{t}^{C}$, with the interaction between the nominal-real covariance and the portfolio-specific component of inflation betas, $\widehat{\beta_{\Pi, p}}=T^{-1} \sum_{t=1}^{T} \beta_{\Pi, p, t}$. Model [4] replaces the interaction term, $\beta_{\Pi, p, t} \times N R C_{t}^{C}$, with the interaction between the nominal-real covariance and the time-specific component of inflation betas, $\widehat{\beta_{\Pi, t}}=10^{-1} \sum_{p=1}^{10} \beta_{\Pi, p, t}$. Model [5] includes both component-wise interaction terms. To accommodate interpretation, $N R C_{t}^{C}$ is standardized in the time series to have mean zero and standard deviation equal to one, whereas $\beta_{\Pi, p, t}$ is demeaned in the pool. The sample period is July 1967 to December 2014.

\begin{tabular}{|c|c|c|c|c|c|c|c|c|}
\hline \multicolumn{6}{|c|}{ Panel A: Coefficient Estimates } & \multicolumn{3}{|c|}{ Panel B: Predicted Risk Premia from [2] } \\
\hline Model & {$[1]$} & {$[2]$} & {$[3]$} & {$[4]$} & {$[5]$} & & High $N R C_{t}^{C}$ & Low $N R C_{t}^{C}$ \\
\hline$L_{0}$ & 8.48 & 5.10 & 8.48 & 4.96 & 5.00 & High $\beta_{\Pi, p, t}$ & 7.65 & -0.50 \\
\hline$t$ & $(3.48)$ & $(2.06)$ & $(3.48)$ & $(1.96)$ & $(1.97)$ & $t$ & $(2.02)$ & $(-0.11)$ \\
\hline$L_{\beta_{\Pi}}$ & 0.51 & -0.51 & 0.56 & -0.62 & -0.57 & $t_{\text {boot }}$ & $(1.65)$ & $(-0.09)$ \\
\hline$t$ & $(0.50)$ & $(-0.65)$ & $(0.56)$ & $(-0.77)$ & $(-0.70)$ & Low $\beta_{\Pi, p, t}$ & -3.33 & 16.56 \\
\hline$L_{N R C}$ & -2.09 & -2.93 & -2.17 & -2.86 & -2.92 & $t$ & $(-0.54)$ & $(4.09)$ \\
\hline$t$ & $(-0.64)$ & $(-1.09)$ & $(-0.66)$ & $(-1.06)$ & $(-1.09)$ & $t_{\text {boot }}$ & $(-0.52)$ & $(3.18)$ \\
\hline$L_{\beta_{\Pi} \times N R C}$ & & 2.36 & & & & Diff & 10.98 & -17.05 \\
\hline$t$ & & $(3.63)$ & & & & $t$ & $(1.90)$ & $(-2.69)$ \\
\hline$L_{\beta_{\Pi, p} \times N R C}$ & & & 2.45 & & 2.17 & $t_{\text {boot }}$ & $(1.40)$ & $(-2.27)$ \\
\hline$t$ & & & $(2.80)$ & & $(2.50)$ & & & \\
\hline$L_{\beta_{\Pi, t} \times N R C}$ & & & & 2.45 & 2.43 & & & \\
\hline$t$ & & & & $(3.45)$ & $(3.42)$ & & & \\
\hline$R^{2}$ & 0.71 & 8.80 & 1.40 & 8.26 & 8.80 & & & \\
\hline
\end{tabular}


Table 7: The Nominal-Real Covariance and the Market Risk Premium (MRP)

Panel A of this table presents coefficient estimates from time-series predictive regressions of CRSP value-weighted excess stock market returns on the market's inflation beta $\left(\beta_{\Pi, m, t}\right)$, the nominal-real covariance $\left(N R C_{t}^{C}\right)$, and their interaction. The right block of results in Panel A presents the predicted MRP from Model [2] in four cases, that is, when $\beta_{\Pi, m, t}$ and $N R C_{t}^{C}$ equal plus or minus one standard deviation from their respective means. We present this regression for the twelve-month horizon and standard errors are Newey-West(12). To accommodate interpretation, $N R C_{t}^{C}$ is standardized and $\beta_{\Pi, m, t}$ is demeaned. Panel $\mathrm{B}$ presents non-parametric estimates of the MRP in four similar cases. These estimates are calculated by averaging realized excess market returns in sample months $t+1$ when $\beta_{\Pi, m, t}$ and $N R C_{t}^{C}$ in month $t$ are either below the $40^{t h}$ or above the $60^{t h}$ percentile. The sample period is July 1967 to December 2014.

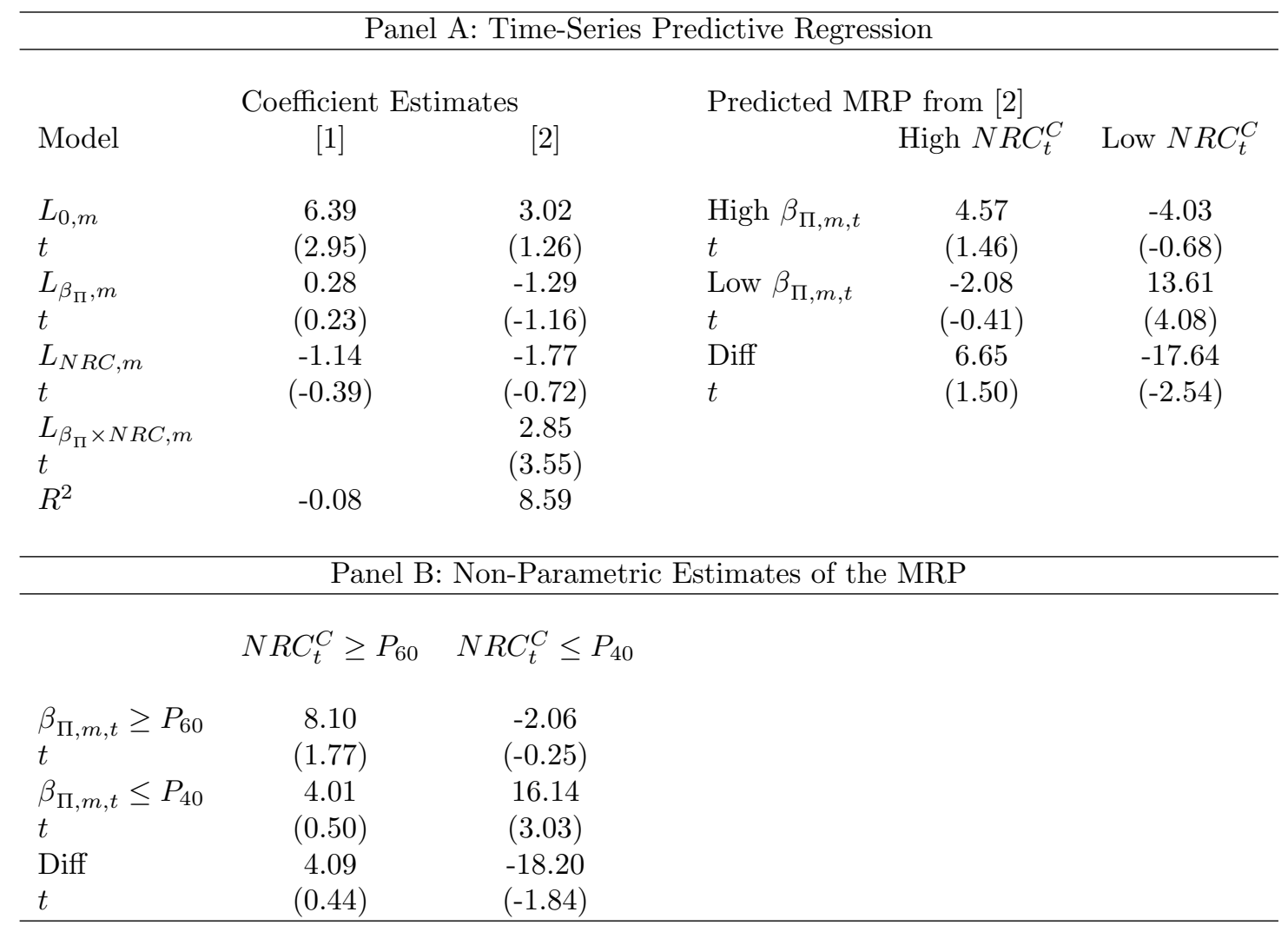


Table 8: Sorting Stocks on Exposure to Inflation-Hedging Industry Factors

For this table, we rank 48 industries on their full sample, unconditional exposure to ARMA $(1,1)$-innovations in inflation $\left(u_{\Pi, t}\right)$. From this ranking, we construct long-short industry factors that equal-weight the top (bottom) $K$ industries, where $K$ ranges from one to five. We then sort individual stocks in decile portfolios on their (60 month rolling window) exposure to these industry factors. We leave out all stocks that belong to the top and bottom $K$ industries, which are presented in Panel A. We then construct a High-minus-Low decile portfolio using the market-value weighted portfolios of the stocks with the highest and lowest exposures to the $K$ th industry factor. For each of these $K$ sorts, we present in Panel B: the ex post, or post-ranking, exposure to $u_{\Pi, t}$; and, the estimates from a predictive regression at the annual horizon of the High-minus-Low portfolio returns on the lagged nominal-real covariance (see Eq. (7) of the paper).

\begin{tabular}{|c|c|c|c|c|c|c|}
\hline & $K$ & 1 & 2 & 3 & 4 & 5 \\
\hline \multicolumn{7}{|c|}{ Panel A: The Five Best and Worst Inflation Hedging Industries } \\
\hline $\begin{array}{l}\text { Top } \\
\text { (Best Hedges) }\end{array}$ & $\begin{array}{l}1 \\
2 \\
3 \\
4 \\
5\end{array}$ & Coal & $\begin{array}{c}\text { Coal } \\
\text { Oil }\end{array}$ & $\begin{array}{l}\text { Coal } \\
\text { Oil } \\
\text { Gold }\end{array}$ & $\begin{array}{l}\text { Coal } \\
\text { Oil } \\
\text { Gold } \\
\text { Mines }\end{array}$ & $\begin{array}{c}\text { Coal } \\
\text { Oil } \\
\text { Gold } \\
\text { Mines } \\
\text { Agriculture }\end{array}$ \\
\hline $\begin{array}{l}\text { Bottom } \\
\text { (Worst Hedges) }\end{array}$ & $\begin{array}{l}1 \\
2 \\
3 \\
4 \\
5\end{array}$ & Meals & $\begin{array}{c}\text { Meals } \\
\text { Textiles }\end{array}$ & $\begin{array}{c}\text { Meals } \\
\text { Textiles } \\
\text { Clothes }\end{array}$ & $\begin{array}{c}\text { Meals } \\
\text { Textiles } \\
\text { Clothes } \\
\text { Transportation }\end{array}$ & $\begin{array}{c}\text { Meals } \\
\text { Textiles } \\
\text { Clothes } \\
\text { Transportation } \\
\text { Personal Svcs. }\end{array}$ \\
\hline \multicolumn{7}{|c|}{ Panel B: Inflation Exposure and Predictability of Industry-Sorted Portfolios } \\
\hline & $\beta_{\Pi, p o s t}$ & $\begin{array}{c}5.18 \\
(4.99)\end{array}$ & $\begin{array}{c}4.70 \\
(4.57)\end{array}$ & $\begin{array}{c}4.41 \\
(4.33)\end{array}$ & $\begin{array}{c}4.27 \\
(4.49)\end{array}$ & $\begin{array}{c}4.21 \\
(4.32)\end{array}$ \\
\hline & $L_{0}$ & $\begin{array}{l}-4.10 \\
(-1.36)\end{array}$ & $\begin{array}{l}-4.97 \\
(-2.17)\end{array}$ & $\begin{array}{c}-5.27 \\
(-2.30)\end{array}$ & $\begin{array}{l}-4.78 \\
(-2.12)\end{array}$ & $\begin{array}{c}-5.05 \\
(-2.20)\end{array}$ \\
\hline & $L_{N R C}$ & $\begin{array}{c}9.03 \\
(3.16)\end{array}$ & $\begin{array}{c}5.86 \\
(2.77)\end{array}$ & $\begin{array}{c}6.01 \\
(3.01)\end{array}$ & $\begin{array}{c}6.26 \\
(3.19)\end{array}$ & $\begin{array}{c}5.64 \\
(2.96)\end{array}$ \\
\hline & $R^{2}$ & 12.13 & 8.72 & 9.15 & 9.94 & 8.04 \\
\hline
\end{tabular}


Table 9: The Inflation Risk Premium Within- and Across-Industry

This table presents time-series predictive regressions for two alternative inflation risk premia (derived from a within- and an across-industry sort) on the lagged nominal-real covariance. For the across-industry sort, we calculate the inflation beta of each of 48 industries as the value-weighted average inflation beta of all stocks in that industry. We sort the industries into quintile portfolios, such that each across-industry quintile portfolio contains either 9 or 10 industries, which we equal-weight to calculate returns. For the within-industry sort, we first construct five value-weighted stock portfolios within each of the 48 industries by splitting at the quintiles of ranked inflation betas of the stocks within that industry. This gives us a total of 48 -by-5 value-weighted portfolios. Within-industry quintile portfolio returns are then calculated as the equal-weighted average over the 48 industries. The aggregate across- and within-industry effects are presented in the last column as the difference between the High and Low across- and within-industry portfolio returns, respectively. For each portfolio, we present coefficient estimates from the predictive regression of twelve-month compounded future returns on the lagged nominal-real covariance (as in Table $5)$.

\begin{tabular}{|c|c|c|c|c|c|c|}
\hline & \multicolumn{6}{|c|}{ Panel A: Across-Industry } \\
\hline & $\begin{array}{c}\text { High } \\
\beta_{\Pi} \text { industries }\end{array}$ & 2.00 & 3.00 & 4.00 & $\begin{array}{c}\text { Low } \\
\beta_{\Pi} \text { industries }\end{array}$ & High-Low \\
\hline$L_{0}$ & 5.93 & 6.99 & 7.94 & 5.78 & 9.29 & -3.36 \\
\hline$t$ & $(2.98)$ & $(3.29)$ & $(3.57)$ & $(2.41)$ & $(3.62)$ & $(-1.95)$ \\
\hline$L_{N R C}$ & 0.69 & -0.67 & -1.23 & -1.51 & -3.94 & 4.63 \\
\hline$t$ & $(0.30)$ & $(-0.28)$ & $(-0.48)$ & $(-0.54)$ & $(-1.30)$ & $(2.70)$ \\
\hline \multirow[t]{3}{*}{$R^{2}$} & -0.02 & -0.05 & 0.22 & 0.38 & 2.95 & 10.03 \\
\hline & \multicolumn{6}{|c|}{ Panel B: Within-Industry } \\
\hline & $\begin{array}{c}\text { High } \\
\beta_{\Pi} \text { stocks }\end{array}$ & 2.00 & 3.00 & 4.00 & $\begin{array}{c}\text { Low } \\
\beta_{\Pi} \text { stocks }\end{array}$ & High-Low \\
\hline$L_{0}$ & 6.56 & 7.16 & 7.78 & 7.89 & 8.28 & -1.73 \\
\hline$t$ & $(2.58)$ & $(2.93)$ & $(3.54)$ & $(3.33)$ & $(3.42)$ & $(-1.55)$ \\
\hline$L_{N R C}$ & 0.27 & 0.15 & -1.09 & -2.10 & -2.97 & 3.24 \\
\hline$t$ & $(0.09)$ & $(0.05)$ & $(-0.41)$ & $(-0.75)$ & $(-1.07)$ & $(3.29)$ \\
\hline$R^{2}$ & -0.17 & -0.17 & 0.14 & 0.86 & 1.74 & 10.81 \\
\hline
\end{tabular}




\section{Table 10: Inflation Risk in Cash Flows Versus Discount Rates}

We use the procedure in Campbell and Vuolteenaho (2004) to decompose the unexpected return of the High and Low inflation beta-sorted portfolios into discount rate and cash flow news: $R_{t+1}-E_{t}\left(R_{t+1}\right)=-N_{D R, t+1}+N_{C F, t+1}$. We regress each of these components separately on $\operatorname{ARMA}(1,1)$-innovations in inflation $\left(u_{\Pi, t}\right)$ to decompose inflation betas: $\beta_{\Pi}=\beta_{\Pi}^{-D R}+\beta_{\Pi}^{C F}$. We consider three sets of state variables. In the first set, we include the term spread $(T S)$, price-earnings ratio $(P E)$, and value spread $(V S)$. The second set includes the dividend yield $(D Y)$, default spread $(D S)$ and $T S$. In the third set, we add the nominal-real covariance $\left(N R C^{C}\right)$ to the first specification. To ensure that the VAR-models are correctly specified, we include in all three specifications the dividend yield of the respective inflation beta-sorted portfolio $\left(D Y_{H / L}\right)$ as a state variable. We report below the estimated exposures. The $t$-statistics use either Newey-West standard errors with one lag $\left(t_{N W}\right)$ or standard errors simulated under the null of no stock return predictability as in Campbell (1991) $\left(t_{\text {sim }}\right)$. In this simulation, we allow (innovations in) the state variables and inflation to be correlated, just like in the data. For each portfolio, we also report the resulting return variance decomposition (column \% Var) that shows the share of return variance explained by (the negative of) discount rate news, cash flow news, and two times their covariance. In the last row, we report the fraction of the High-minus-Low difference in inflation beta coming from exposure in cash flows.

\begin{tabular}{|c|c|c|c|c|c|c|c|c|c|c|c|c|c|}
\hline & \multicolumn{4}{|c|}{ Model 1: TS,PE,VS,DY $Y_{H / L}$} & \multicolumn{4}{|c|}{ Model 2: $D Y_{M K T}, D S, T S, D Y_{H / L}$} & \multicolumn{4}{|c|}{ Model 3: $N R C^{C}, T S, P E, V S, D Y_{H / L}$} \\
\hline & & $\beta_{\Pi}$ & $t_{N W}$ & $t_{\text {sim }}$ & $\% \operatorname{Var}$ & $\beta_{\Pi}$ & $t_{N W}$ & $t_{\text {sim }}$ & $\%$ Var & $\beta_{\Pi}$ & $t_{N W}$ & $t_{s i m}$ & $\% \operatorname{Var}$ \\
\hline \multirow[t]{4}{*}{ High } & $R_{t+1}-E_{t}\left[R_{t+1}\right]$ & -0.89 & $(-0.54)$ & $(-0.83)$ & 1.00 & -0.94 & $(-0.57)$ & $(-0.90)$ & 1.00 & -0.87 & $(-0.52)$ & $(-0.81)$ & 1.00 \\
\hline & $-N_{D R, t+1}$ & -1.78 & $(-1.57)$ & $(-1.94)$ & 0.43 & -1.66 & $(-1.32)$ & $(-1.06)$ & 0.57 & -2.82 & $(-2.14)$ & $(-2.84)$ & 0.87 \\
\hline & $N_{C F, t+1}$ & 0.89 & $(1.24)$ & $(0.66)$ & 0.34 & 0.71 & $(1.01)$ & $(0.39)$ & 0.32 & 1.95 & $(1.67)$ & $(1.40)$ & 1.08 \\
\hline & $2 \times \operatorname{Cov}\left(-N_{D R, t+1}, N_{C F, t+1}\right)$ & & & & 0.23 & & & & 0.11 & & & & -0.95 \\
\hline \multirow[t]{5}{*}{ Low } & $R_{t+1}-E_{t}\left[R_{t+1}\right]$ & -2.71 & $(-1.65)$ & $(-2.58)$ & 1.00 & -2.73 & $(-1.66)$ & $(-2.60)$ & 1.00 & -2.70 & $(-1.65)$ & $(-2.57)$ & 1.00 \\
\hline & $-N_{D R, t+1}$ & -1.20 & $(-1.66)$ & $(-1.29)$ & 0.37 & -2.02 & $(-3.73)$ & $(-0.17)$ & 0.37 & -1.05 & $(-1.40)$ & $(-0.88)$ & 0.35 \\
\hline & $N_{C F, t+1}$ & -1.51 & $(-1.45)$ & $(-1.15)$ & 0.42 & -0.71 & $(-0.48)$ & $(-0.06)$ & 0.62 & -1.65 & $(-1.70)$ & $(-1.13)$ & 0.39 \\
\hline & $2 \times \operatorname{Cov}\left(-N_{D R, t+1}, N_{C F, t+1}\right)$ & & & & 0.22 & & & & 0.02 & & & & 0.26 \\
\hline & $\frac{\beta_{\Pi, H i g h}^{C F}-\beta_{\Pi, L o w}^{C F}}{\beta_{\Pi, H i g h}-\beta_{\Pi, L o w}}$ & 1.32 & & & & 0.80 & & & & 1.96 & & & \\
\hline
\end{tabular}


Table 11: Calibrated Parameters of the Model

This table reports the configuration of the parameters used in the calibration of the model. The model is calibrated on a monthly decision interval.

\begin{tabular}{|c|c|c|}
\hline \multicolumn{3}{|l|}{ Preferences } \\
\hline Discount factor & $\delta$ & 0.99 \\
\hline Elasticity of intertemporal substitution & $\psi$ & 2 \\
\hline Risk aversion coefficient & $\gamma$ & 15 \\
\hline \multicolumn{3}{|l|}{ Inflation } \\
\hline Mean of inflation rate & $\mu_{\pi}$ & 0.0033 \\
\hline Inflation AR(1) coefficient & $\rho_{\pi}$ & 0.7992 \\
\hline \multirow[t]{2}{*}{ Inflation MA(1) coefficients } & $\phi_{\pi}$ & -0.0025 \\
\hline & $\xi_{\pi}$ & $7.30 \times 10^{-4}$ \\
\hline \multicolumn{3}{|l|}{ Consumption } \\
\hline Mean of real consumption growth & $\mu_{c}$ & 0.0016 \\
\hline Exposure of expected consumption to inflation & $\rho_{c}$ & -0.0521 \\
\hline Volatility of consumption-specific shocks & $\sigma_{c}$ & 0.0020 \\
\hline Consumption exposure to inflation shocks & $\xi_{c}$ & -0.1983 \\
\hline \multicolumn{3}{|l|}{ Nominal-real covariance } \\
\hline Mean of nominal-real covariance & $\varphi_{0}$ & -0.0062 \\
\hline Persistence of nominal-real covariance & $\nu$ & 0.9963 \\
\hline Volatility of nominal-real covariance & $\sigma_{w}$ & 0.0029 \\
\hline \multicolumn{3}{|l|}{ High inflation beta portfolio } \\
\hline Mean of dividend growth & $\mu_{H}$ & $3.44 \times 10^{-4}$ \\
\hline Exposure of expected dividends to inflation & $\rho_{H}$ & -0.2345 \\
\hline Exposure to consumption-specific shocks & $\sigma_{H}$ & 0.0318 \\
\hline Exposure to inflation shocks & $\xi_{H}$ & 0.1942 \\
\hline \multicolumn{3}{|l|}{ Low inflation beta portfolio } \\
\hline Mean of dividend growth & $\mu_{L}$ & 0.0060 \\
\hline Exposure of expected dividends to inflation & $\rho_{L}$ & 0.6989 \\
\hline Exposure to consumption-specific shocks & $\sigma_{L}$ & -0.0517 \\
\hline Exposure to inflation shocks & $\xi_{L}$ & 0.1082 \\
\hline \multicolumn{3}{|l|}{ Aggregate Market } \\
\hline Mean of dividend growth & $\mu_{M K T}$ & $1.72 \times 10^{-4}$ \\
\hline Exposure of expected dividends to inflation & $\rho_{M K T}$ & 0.7099 \\
\hline Exposure to consumption-specific shocks & $\sigma_{M K T}$ & 0.0337 \\
\hline Exposure to inflation shocks & $\xi_{M K T}$ & -0.3076 \\
\hline
\end{tabular}


Table 12: Calibrated Moments

This table shows that the model can match means and standard deviations of returns and betas, as well as the slope coefficient from regressing returns on the High-minus-Low portfolio (as well as the aggregate market portfolio) on the nominal-real covariance.

\begin{tabular}{|c|c|c|c|c|c|c|}
\hline & \multicolumn{3}{|c|}{ Data } & \multicolumn{3}{|c|}{ Model } \\
\hline \multicolumn{7}{|c|}{ Descriptive Statistics } \\
\hline & $\pi_{t}$ & $u_{\pi, t}$ & $\Delta c_{t}$ & $\pi_{t}$ & $u_{\pi, t}$ & $\Delta c_{t}$ \\
\hline Mean & 3.93 & 0.11 & 1.97 & 3.93 & 0.00 & 1.97 \\
\hline St. dev. & 1.11 & 0.85 & 1.13 & 1.11 & 0.85 & 2.49 \\
\hline $\operatorname{AR}(1)$ & 62.47 & 15.35 & -16.84 & 62.47 & 0.00 & 0.19 \\
\hline \multicolumn{7}{|l|}{ Correlations } \\
\hline$\pi_{t}$ & 1 & 79.03 & -17.75 & 1 & 76.71 & -8.10 \\
\hline$u_{\pi, t}$ & & 1 & -17.41 & & 1 & 0.00 \\
\hline$\Delta c_{t}$ & & & 1 & & & 1 \\
\hline \multicolumn{7}{|c|}{ Predictve Regressions of Consumption Growth on Inflation } \\
\hline & Uncond. & Cond. & & Uncond. & Cond. & \\
\hline$d_{0}^{12}$ & 0.02 & 0.00 & & 0.02 & 0.00 & \\
\hline$d_{1}^{12}$ & -0.50 & 0.81 & & -0.50 & 1.00 & \\
\hline$R^{2}$ & 1.12 & 14.92 & & 0.41 & 15.40 & \\
\hline $\operatorname{AR}(1) N R C_{t}^{C}$ & & 99.63 & & & 99.63 & \\
\hline St. dev. $N R C_{t}^{C}$ & & 0.87 & & & 0.79 & \\
\hline \multicolumn{7}{|c|}{ Characterizing Inflation Beta-Sorted Portfolios } \\
\hline & High & Low & & High & Low & \\
\hline Mean $\beta_{u, i t}$ & -0.02 & -3.02 & & -0.02 & -3.04 & \\
\hline St. dev. $\beta_{u, i t}$ & 3.61 & 3.25 & & 4.03 & 2.86 & \\
\hline Mean Ret. & 5.26 & 9.49 & & 5.28 & 9.42 & \\
\hline St. dev. & 22.72 & 21.78 & & 22.74 & 18.97 & \\
\hline \multicolumn{7}{|c|}{ Characterizing Inflation Risk Premium $\left(I P_{t}^{H L}\right)$} \\
\hline St. $\operatorname{dev} \beta_{u, H L t}$ & & 1.36 & & & 1.00 & \\
\hline$L_{N R C, I P_{t}^{H L}}$ & & 5.38 & & & 5.41 & \\
\hline \multicolumn{7}{|c|}{ The NRC and the Market Risk Premium } \\
\hline Mean $\beta_{u, m t}$ & & -1.96 & & & -1.95 & \\
\hline St. dev. $\beta_{u, m t}$ & & 2.19 & & & 2.85 & \\
\hline $\operatorname{corr}\left(\beta_{u, H}, \beta_{u, m}\right)$ & & 95.75 & & & 100.00 & \\
\hline $\operatorname{corr}\left(\beta_{u, L}, \beta_{u, m}\right)$ & & 97.12 & & & 100.00 & \\
\hline$L_{\beta_{\Pi} \times N R C, m}$ & & 2.85 & & & 2.69 & \\
\hline
\end{tabular}




\section{Online Appendix to "Time-Varying Inflation Risk and Stock Returns"}

In this Online Appendix, we present more detail for the block-bootstrap procedure as well as the derivation of our model. We finally report results from a variety of robustness checks.

\section{Bootstrap Algorithm}

The block-bootstrap algorithm associated to the regressions of Tables 2, 5 and 6 in the paper consists of the following steps.

1. In each replication $m=1, \ldots, 1000$, we construct pseudo-samples for both consumption growth and inflation by drawing with replacement $T_{m}$ overlapping two-year blocks from:

$$
\left\{\Delta C_{t+1: t+24}^{m}, \Pi_{t+1: t+24}^{m}\right\}, t=s_{1}^{m}, s_{2}^{m}, \ldots, s_{T_{m}}^{m}
$$

where the time indices, $s_{1}^{m}, s_{2}^{m}, \ldots, s_{T_{m}}^{m}$, are drawn randomly from the original time sequence $1, \ldots, T$. The two-year block size is chosen to preserve the (auto-) correlation between consumption growth and inflation in the data and to respect the estimation setup in Eqs. (2) and (3) of the paper. Additionally, it is a way to conserve the size of the cross-section in the resampled CRSP file (see Step 3 below). We join these blocks to construct a monthly time series matching the length of the sample from July 1967 to December 2014.

2. For $m=1, \ldots, 1000$, we run the two-stage tests described in Section 3. for the artificial data:

$$
\begin{aligned}
& \Delta C_{t+1: t+K}^{m}=d_{m, 0}^{c, K}+d_{m, 1}^{c, K}\left(\widehat{a_{m, t}^{K}}+\widehat{b_{m, t}^{K}} \Pi_{t}^{m}\right)+e_{t+1: t+K}^{m}, \text { where } \\
& \Delta C_{s+1: s+K}^{m} \quad=a_{m, t}^{K}+b_{m, t}^{K} \Pi_{s}^{m}+e_{s+1: s+K}^{m}, s=1, \ldots, t-K
\end{aligned}
$$

and save the estimates $d_{m, 0}^{c, K}, d_{m, 1}^{c, K}$, and $b_{m, t}^{K}$, for $K=1,3,6,12$. The bootstrapped standard errors reported in Table 2 are calculated as the standard deviation of $d_{m, 0}^{c, K}$ and $d_{m, 1}^{c, K}$ over the 1000 bootstrap replications. The bootstrap estimates, $b_{m, t}^{12}$, represents the artificial nominal-real covariance that is going to be used to get the bootstrapped standard errors for the remaining tables of the paper.

3. Using the same time indexes $s_{1}^{m}, s_{2}^{m}, \ldots, s_{T_{m}}^{m}$, we re-sample all firms $i=1, \ldots, I$ in the CRSP file. To be consistent with the data, we bootstrap both returns, $R_{t+1}=\left\{R_{1, t+1}, R_{2, t+1}, \ldots, R_{I, t+1}\right\}^{\prime}$, and firm characteristics, $Z_{t}=\left\{M V_{t}, B M_{t}, M O M_{t}\right\}$, with, e.g., $M V_{t}=\left\{M V_{1, t}, M V_{2, t}, \ldots, M V_{I, t}\right\}^{\prime}$, such that:

$$
\left\{R_{t+1: t+24}^{m}, Z_{t: t+24-1}^{m}\right\}, t=s_{1}^{m}, s_{2}^{m}, \ldots, s_{T_{m}}^{m} .
$$


Notice that the characteristics are lagged by one month just like in the data. We join these blocks to construct 1000 artificial CRSP files matching the length of our sample.

4. In each replication, we estimate at the end of month $t$ and for each artificial stock $i$ its exposure to $\operatorname{ARMA}(1,1)$-innovations in inflation, denoted $u_{\Pi, t+1}^{m}$. The ARMA model is estimated for the inflation series described in Step 1. The inflation betas are estimated using the WLS-Vasicek procedure described in Section 4.1.1. of the paper. We require that an artificial stock return series has at least 24 out of the last 60 months of returns available to estimate inflation beta, $\beta_{\Pi, i, t}^{m}$. Since many stocks have some missing returns in the CRSP file, due to late introduction or early exit, the overlapping block-bootstrap reduces the number of firms that satisfy this requirement relative to the data. However, we end up with about two-thirds of the number of firms that we use in the data in each bootstrapped cross-section.

5. For $m=1, \ldots, 1000$ and at the end of each month $t$, we then sort the artificial stocks on these inflation betas and their market values to construct the ten value-weighted size-controlled inflation beta-sorted portfolios that feature prominently in the paper, $R_{p, t+1}^{m}=\left\{R_{H i g h, t+1}^{m}, R_{2, t+1}^{m}, \ldots, R_{\text {Low,t+1 }}^{m}\right\}$. The three bootstrap estimates of the inflation risk premium are constructed as follows. First, we take the highminus-low spreading portfolio from this sort: $R_{I P_{t}^{H L}, t+1}^{m}=R_{H i g h, t+1}^{m}-R_{L o w, t+1}^{m}$. Second, we regress the artificial $\operatorname{ARMA}(1,1)$-innovations in inflation, $u_{\Pi, t+1}^{m}$, on the inflation sorted portfolios to construct the maximum correlation inflation-mimicking portfolio:

$$
u_{\Pi, t+1}^{m}=\text { intercept }_{m}+\text { weights } s_{m}^{\prime} \times R_{p, t+1}^{m}+e_{t+1}^{m},
$$

such that $R_{I P_{t}^{M C}, t+1}^{m}$ is the portfolio return weights ${ }_{m}^{\prime} \times R_{p, t+1}^{m}$. Finally, we run a cross-sectional regression of returns on lagged inflation betas, where we control for the firm characteristics:

$$
R_{i, t+1}^{m}=l_{m, 0, t}+l_{m, \Pi, t} \beta_{\Pi, i, t}^{m}+l_{Z, t} Z_{n, t}^{m}+u_{t+1}^{m} .
$$

The time series of coefficient estimates, $l_{m, \Pi, t}$, represents our third estimate of the inflation risk premium $R_{I P_{t}^{C S}, t+1}^{m}$.

6. For each replication, we then run the predictive regression described in Section 4.2. of the paper. That is, we regress returns on the artificial inflation portfolios and risk premia (compounded over horizons $K=1,3,12$ months) on the lagged nominal-real covariance (i.e., the bootstrap coefficient estimate $b_{m, t}^{12}$ from Step 2 above) using:

$$
R_{p, t+1: t+K}^{m}=L_{m, 0}+L_{m, N R C} b_{m, t}^{12}+\varepsilon_{t+1: t+K}^{m}
$$

Note that the timing in the different steps of the bootstrap is consistent with the data, so that the lefthand side returns are observed strictly after the consumption and inflation numbers used to estimate the right-hand side nominal-real covariance. We use the standard deviation of the estimates $L_{m, 0}$ and 
$L_{m, N R C}$ over the 1000 bootstrap replications as the standard error for the predictive regressions of Tables 5 .

7. Finally, we run the pooled predictive regressions described in Section 5.1. of the paper. That is, we regress returns on the artificial inflation beta-sorted decile portfolios, $p=H i g h, 2,3, \ldots$, Low on their time-varying inflation betas $\left(\beta_{\Pi, p, t}^{m}\right)$, the nominal-real covariance, and an interaction:

$$
R_{p, t+1: t+K}^{m}=L_{m, 0}+L_{m, \beta_{\Pi}} \beta_{\Pi, p, t}^{m}+L_{m, N R C} b_{m, t}^{12}+L_{m, \beta_{\Pi} \times N R C}\left(\beta_{\Pi, p, t}^{m} \times b_{m, t}^{12}\right)+\varepsilon_{t+1: t+K}^{m}
$$

Because this regression includes an interaction term, the bootstrap may contain samples $m$ that suffer from multicollinearity. To address this problem, we evaluate the joint significance of the variables by performing inference on the predicted risk premia when $\beta_{\Pi, p, t}^{m}$ and $b_{m, t}^{12}$ are at plus or minus one standard deviation from their respective means in the block-bootstrapped sample. We use the standard deviation of these predicted risk premia to calculate the $t$-statistics for the estimates report in Panel B of Table 6 .

\section{Model Derivations}

This section present detailed derivations of our model results, including expressions for the moments that we target in our calibration.

\subsection{Setup}

The representative agent has preferences given by the recursive utility function of Epstein and Zin (1989) and Kreps and Porteus (1978),

$$
U_{t}\left(W_{t}\right)=\left((1-\delta) C_{t}^{1-1 / \psi}+\delta E_{t}\left[U_{t+1}\left(W_{t+1}\right)^{1-\gamma}\right]^{\frac{1-1 / \psi}{1-\gamma}}\right)^{\frac{1}{1-1 / \psi}}
$$

where $W_{t}$ is real aggregate wealth and $C_{t}$ is real aggregate consumption. The constant $\delta \in(0,1)$ is the discount rate, $\gamma>0$ is the coefficient of relative risk aversion and $\psi>0$ is the elasticity of intertemporal substitution (EIS). The first order condition for the representative agent's problem implies that the gross return $R_{i, t+1}$ on any tradable asset $i$ satisfies the Euler equation

$$
1=E_{t}\left[M_{t+1} R_{i, t+1}\right]
$$

with a stochastic discount factor $M_{t+1}$ given by

$$
m_{t+1} \equiv \log M_{t+1}=\theta \log \delta-\frac{\theta}{\psi} \Delta c_{t+1}+(\theta-1) r_{c, t+1},
$$

where

$$
\theta=\frac{1-\gamma}{1-\frac{1}{\psi}}
$$


and lowercase letters denote logarithms, so that $\Delta c_{t}=\ln C_{t}-\ln C_{t-1}$ and $r_{c, t}=\log \left(R_{c, t}\right)$. The processes for real consumption growth, $\Delta c_{t}$, inflation, $\pi_{t}$, the nominal-real covariance, $\varphi_{t}$, and real dividend growth for asset $i, \Delta d_{i, t}$, are exogenous and given by

$$
\begin{aligned}
\pi_{t+1}= & \mu_{\pi}+\rho_{\pi}\left(\pi_{t}-\mu_{\pi}\right)+\phi_{\pi} u_{t+1}+\xi_{\pi} u_{t}, \\
\Delta c_{t+1}= & \mu_{c}+\rho_{c}\left(\pi_{t}-\mu_{\pi}\right)+\sigma_{c} \eta_{t+1}+\xi_{c} \varphi_{t-1} u_{t}, \\
\varphi_{t+1}= & \varphi_{0}+v\left(\varphi_{t}-\varphi_{0}\right)+\sigma_{w} w_{t+1}, \\
\Delta d_{i, t+1}= & \mu_{i}+\rho_{i}\left(\pi_{t}-\mu_{\pi}\right)+\sigma_{i} \eta_{t+1}+\xi_{i} \varphi_{t-1} u_{t}, \\
& u_{t}, \eta_{t}, w_{t} \text { iid standard normal. }
\end{aligned}
$$

\subsection{The Nominal-Real Covariance}

The covariance between time- $(t+1)$ inflation and consumption conditional on times $t$ and $t-1$ are

$$
\begin{aligned}
\operatorname{Cov}_{t}\left(\Delta c_{t+1}, \pi_{t+1}\right) & =0 \\
\operatorname{Cov}_{t-1}\left(\Delta c_{t+1}, \pi_{t+1}\right) & =\phi_{\pi} \rho_{c}\left(\xi_{\pi}+\phi_{\pi} \rho_{\pi}\right)+\xi_{c}\left(\rho_{\pi} \phi_{\pi}+\xi_{\pi}\right) \varphi_{t-1} .
\end{aligned}
$$

Conditional predictability of consumption growth with inflation is given by

$$
\operatorname{Cov}_{t}\left(\Delta c_{t+1+j}, \pi_{t+1}\right)=\left\{\begin{array}{cc}
\phi_{\pi}^{2} \rho_{c}+\phi_{\pi} \xi_{c} \varphi_{t} & , \text { if } j=1 \\
\phi_{\pi} \rho_{c} \rho_{\pi}^{j-2}\left(\xi_{\pi}+\phi_{\pi} \rho_{\pi}\right) & , \text { if } j>1
\end{array},\right.
$$

and, using that

$$
\operatorname{Var}_{t}\left(\pi_{t+1}\right)=\phi_{\pi}^{2}
$$

we get

$$
\begin{aligned}
b_{t}^{K} & =\frac{\operatorname{Cov}_{t}\left(\sum_{j=1}^{K} \Delta c_{t+1+j}, \pi_{t+1}\right)}{\operatorname{Var}_{t}\left(\pi_{t+1}\right)} \\
& =\left(1+\left(\frac{\xi_{\pi}}{\phi_{\pi}}+\rho_{\pi}\right) \frac{\rho_{\pi}^{K-1}-1}{\rho_{\pi}-1}\right) \rho_{c}+\frac{\xi_{c}}{\phi_{\pi}} \varphi_{t} \\
& =h_{K}+\frac{\xi_{c}}{\phi_{\pi}} \varphi_{t},
\end{aligned}
$$

where we have defined

$$
h_{K} \equiv\left(1+\left(\frac{\xi_{\pi}}{\phi_{\pi}}+\rho_{\pi}\right) \frac{\rho_{\pi}^{K-1}-1}{\rho_{\pi}-1}\right) \rho_{c} .
$$

Our main measure of the nominal-real covariance is

$$
N R C_{t}^{C} \equiv b_{t}^{12}
$$




\subsection{Coefficients of the Wealth-Consumption Ratio}

To price assets, we conjecture (and later verify) that the log wealth-consumption ratio is linear quadratic in the state variables $\pi_{t}, \varphi_{t}$, and $u_{t}$ and has the following form

$$
w c_{t}=A_{0}+A_{1}\left(\pi_{t}-\mu_{\pi}\right)+A_{2} \varphi_{t-1} u_{t}+A_{3} u_{t}+A_{4}\left(\varphi_{t}-\varphi_{0}\right)+A_{5}\left(\varphi_{t}^{2}-E\left[\varphi_{t}^{2}\right]\right)
$$

and that the price-dividend ratio for asset $i$ is

$$
p d_{i, t}=D_{i, 0}+D_{i, 1}\left(\pi_{t}-\mu_{\pi}\right)+D_{i, 2} \varphi_{t-1} u_{t}+D_{i, 3} u_{t}+D_{i, 4}\left(\varphi_{t}-\varphi_{0}\right)+D_{i, 5}\left(\varphi_{t}^{2}-E\left[\varphi_{t}^{2}\right]\right)
$$

where

$$
E\left[\varphi_{t}^{2}\right]=\varphi_{0}^{2}+\frac{\sigma_{w}^{2}}{1-v^{2}}
$$

is the unconditional mean of $\varphi_{t}^{2}$. A Campbell-Shiller approximation gives returns on the aggregate consumption claim (the wealth portfolio), $r_{c, t+1}$, and returns on any asset $i, r_{i, t+1}$,

$$
\begin{aligned}
r_{c, t+1} & =\kappa_{0}+\kappa_{1} w c_{t+1}-w c_{t}+\Delta c_{t+1}, \\
r_{i, t+1} & =\kappa_{i, 0}+\kappa_{i, 1} p d_{i, t+1}-p d_{i, t}+\Delta d_{i, t+1}
\end{aligned}
$$

with approximations constants

$$
\begin{aligned}
\kappa_{1} & =\frac{e^{E\left[w c_{t}\right]}}{e^{E\left[w c_{t}\right]}+1}, \\
\kappa_{0} & =\log \left(e^{E\left[w c_{t}\right]}+1\right)-\frac{e^{E\left[w c_{t}\right]}}{e^{E\left[w c_{t}\right]}+1} E\left[w c_{t}\right], \\
\kappa_{i, 1} & =\frac{e^{E\left[p d_{i, t}\right]}}{e^{E\left[p d_{i, t}\right]}+1}, \\
\kappa_{i, 0} & =\log \left(e^{E\left[p d_{i, t}\right]}+1\right)-\frac{e^{E\left[p d_{i, t}\right]}}{e^{E\left[p d_{i, t}\right]}+1} E\left[p d_{i, t}\right] .
\end{aligned}
$$

Using equations (OA.20), and (OA.9), we get

$$
m_{t+1}+r_{c, t+1}=\theta\left(\log \delta+\kappa_{0}\right)+\theta\left(1-\frac{1}{\psi}\right) \Delta c_{t+1}+\theta\left(\kappa_{1} w c_{t+1}-w c_{t}\right) .
$$

The Euler equation for any asset $i$ (including $i=c$ ) with lognormal returns is

$$
0=E_{t}\left[m_{t+1}+r_{i, t+1}\right]+\frac{1}{2} \operatorname{Var}_{t}\left[m_{t+1}+r_{i, t+1}\right]
$$

Plugging equation (OA.26) into (OA.27) with $i=c$, and evaluating conditional means and variances, the Euler equation for the consumption claim $(i=c)$ can be expressed as

$$
0=C_{0}+C_{1}\left(\pi_{t}-\mu_{\pi}\right)+C_{2} \varphi_{t-1} u_{t}+C_{3} u_{t}+C_{4}\left(\varphi_{t}-\varphi_{0}\right)+C_{5}\left(\varphi_{t}^{2}-E\left[\varphi_{t}^{2}\right]\right)
$$


where $C_{i}$ are constants that depend on $A_{0}, A_{1}, A_{2}, A_{3}, A_{4}, A_{5}$. In order for the Euler equation to be satisfied at all times, the coefficients $C_{i}$ must be identically zero, which yields the following system of equations in $A_{0}, A_{1}, A_{2}, A_{3}, A_{4}, A_{5}$ :

$$
\begin{aligned}
\left(\pi_{t}-\mu_{\pi}\right): \quad 0=\left(1-\frac{1}{\psi}\right) \rho_{c}-A_{1}\left(1-\kappa_{1} \rho_{\pi}\right) \\
\left(\varphi_{t-1} u_{t}\right): \quad 0=\left(1-\frac{1}{\psi}\right) \xi_{c}-A_{2}, \\
\left(u_{t}\right): \quad 0=\kappa_{1} \xi_{\pi} A_{1}-A_{3}, \\
\left(\varphi_{t}-\varphi_{0}\right): \quad 0=\theta \kappa_{1}^{2} A_{2}\left(A_{3}+\phi_{\pi} A_{1}\right)+\left(v \kappa_{1}-1\right) A_{4}, \\
+\left(2 v \theta \kappa_{1}^{2} \sigma_{w}^{2}\right) A_{4} A_{5}-2 \kappa_{1} \varphi_{0} v(v-1)\left(2 \theta \kappa_{1} \sigma_{w}^{2} A_{5}+1\right) A_{5} \\
\left(\varphi_{t}^{2}-E\left[\varphi_{t}^{2}\right]\right): \quad 0=\frac{\theta \kappa_{1}^{2}}{2}\left(A_{2}^{2}+4 v^{2} \sigma_{w}^{2} A_{5}^{2}\right)-A_{5}\left(1-v^{2} \kappa_{1}\right) \\
(\text { const }): \quad 0=\frac{\theta^{2}}{2}\left(\frac{1}{\psi}-1\right)^{2} \sigma_{c}^{2}-\frac{\theta}{\psi} \mu_{c}+\theta\left(\kappa_{1}-1\right) A_{0}+\theta\left(\kappa_{0}+\mu_{c}+\ln \delta\right) \\
\quad+\frac{1}{2} \theta^{2} \kappa_{1}^{2}\left(\left(A_{3}+\varphi_{0} A_{2}+\phi_{\pi} A_{1}\right)^{2}+\sigma_{w}^{2}\left(\left(A_{4}+2 \varphi_{0} A_{5}\right)^{2}+2 \sigma_{w}^{2} A_{5}^{2}\right)\right) \\
\quad+\frac{\theta^{2} \kappa_{1}^{2} \sigma_{w}^{2}}{2\left(1-v^{2}\right)}\left(A_{2}^{2}+4 v^{2} \sigma_{w}^{2} A_{5}^{2}\right) .
\end{aligned}
$$

We solve for $A_{1}, A_{2}, A_{3}, A_{4}, A_{5}$ in terms of $A_{0}$

$$
\begin{aligned}
& A_{1}=\left(1-\frac{1}{\psi}\right) \frac{\rho_{c}}{1-\kappa_{1} \rho_{\pi}}, \\
& A_{2}=\left(1-\frac{1}{\psi}\right) \xi_{c} \\
& A_{3}=\left(1-\frac{1}{\psi}\right) \frac{\kappa_{1} \xi_{\pi} \rho_{c}}{1-\kappa_{1} \rho_{\pi}}, \\
& A_{4}=\frac{A_{2} \theta \kappa_{1}^{2}\left(A_{1} \phi_{\pi}+A_{3}\right)+2 \kappa_{1} \varphi_{0} v(1-v)\left(1+2 \theta \kappa_{1} \sigma_{w}^{2} A_{5}\right) A_{5}}{1-\kappa_{1} v\left(1+2 \theta \kappa_{1} \sigma_{w}^{2} A_{5}\right)}, \\
& A_{5}=\frac{1-v^{2} \kappa_{1}}{\theta\left(2 v \kappa_{1} \sigma_{w}\right)^{2}}\left(1-\sqrt{1-\left(\frac{2(1-\gamma) v \kappa_{1}^{2} \sigma_{w} \xi_{c}}{1-v^{2} \kappa_{1}}\right)^{2}}\right),
\end{aligned}
$$

where we pick the negative root in the expression for $A_{5}$ (which comes from solving a quadratic equation) so that $\xi_{c}=0$ implies the intuitive economic restriction that the nominal-real covariance $\varphi_{t}$ has no effect on the wealth-consumption ratio when it does not affect consumption. Then we solve for $A_{0}$ numerically using equations (OA.23), (OA.22) and (OA.33).

\subsection{Coefficients of the Price-Dividend Ratio}

The calculation is analogous to that for the $A_{i}$ but instead of using the Euler equation for $r_{c, t}$, we use the one for $r_{i, t}$. The Euler equation for asset $i$ can be expressed as

$$
0=F_{i, 0}+F_{i, 1}\left(\pi_{t}-\mu_{\pi}\right)+F_{i, 2} \varphi_{t-1} u_{t}+F_{i, 3} u_{t}+F_{i, 4}\left(\varphi_{t}-\varphi_{0}\right)+F_{i, 5}\left(\varphi_{t}^{2}-E\left[\varphi_{t}^{2}\right]\right)
$$


where $F_{i, j}$ are constants that depend on $D_{i, 0}, D_{i, 1}, D_{i, 2}, D_{i, 3}, D_{i, 4}, D_{i, 5}$. In order for the Euler equation to be satisfied, the coefficients $F_{i, j}$ must be identically zero, which yields the following system of five equations in the five unknowns $D_{i, 1}, D_{i, 2}, D_{i, 3}, D_{i, 4}, D_{i, 5}$

$$
\begin{aligned}
&\left(\pi_{t}-\mu_{\pi}\right): \quad 0=\rho_{i}-D_{i, 1}\left(1-\kappa_{i, 1} \rho_{\pi}\right)+(\theta-1)\left(\rho_{c}+A_{1}\left(\kappa_{1} \rho_{\pi}-1\right)\right)-\frac{\theta}{\psi} \rho_{c}, \\
&\left(\varphi_{t-1} u_{t}\right): \quad 0=\xi_{i}-D_{i, 2}+(\theta-1)\left(\xi_{c}-A_{2}\right)-\frac{\theta}{\psi} \xi_{c}, \\
&\left(u_{t}\right): \quad 0=(\theta-1)\left(\kappa_{1} \xi_{\pi} A_{1}-A_{3}\right)+\xi_{\pi} \kappa_{i, 1} D_{i, 1}-D_{i, 3}, \\
&\left(\varphi_{t}-\varphi_{0}\right): \quad 0=2 D_{i, 5} v \kappa_{i, 1} \varphi_{0}(1-v)-D_{i, 4}\left(1-v \kappa_{i, 1}\right) \\
&-\left(A_{4}-\kappa_{1} v\left(A_{4}+2 A_{5} \varphi_{0}(1-v)\right)\right)(\theta-1) \\
&+\left(\kappa_{i, 1} D_{i, 2}+\kappa_{1} A_{2}(\theta-1)\right)\left(\kappa_{i, 1} D_{i, 3}+\kappa_{1} A_{3}(\theta-1)\right) \\
&+\phi_{\pi}\left(\kappa_{i, 1} D_{i, 2}+\kappa_{1} A_{2}(\theta-1)\right)\left(\kappa_{i, 1} D_{i, 1}+\kappa_{1} A_{1}(\theta-1)\right) \\
&+2 v \sigma_{w}^{2}\left(\kappa_{i, 1} D_{i, 4}+\kappa_{1} A_{4}(\theta-1)\right)\left(\kappa_{i, 1} D_{i, 5}+\kappa_{1} A_{5}(\theta-1)\right) \\
&-4 v \varphi_{0} \sigma_{w}^{2}\left(\kappa_{i, 1} D_{i, 5}+\kappa_{1} A_{5}(\theta-1)\right)^{2}(v-1), \\
& \quad 0=\left(2 v^{2} \sigma_{w}^{2} \kappa_{i, 1}^{2}\right) D_{i, 5}^{2} \\
&+\left(v^{2} \kappa_{i, 1}\left(4 \kappa_{1} \sigma_{w}^{2} A_{5}(\theta-1)+1\right)-1\right) D_{i, 5} \\
&+\frac{1}{2}\left(\kappa_{i, 1} D_{i, 2}-\kappa_{1} A_{2}+\theta \kappa_{1} A_{2}\right)^{2} \\
&+A_{5}(\theta-1)\left(v^{2} \kappa_{1}\left(2 \kappa_{1} \sigma_{w}^{2} A_{5}(\theta-1)+1\right)-1\right),
\end{aligned}
$$


and one equation for $D_{i, 0}$

$$
\begin{aligned}
\left(1-\kappa_{i, 1}\right) D_{i, 0}= & \kappa_{i, 0}+\mu_{i}+\theta \ln \delta-\frac{\theta}{\psi} \mu_{c}+(\theta-1)\left(\kappa_{0}+\mu_{c}-A_{0}\left(1-\kappa_{1}\right)\right) \\
& +\frac{1}{2}\left(\sigma_{i}+\sigma_{c}\left(\theta-\frac{\theta}{\psi}-1\right)\right)^{2} \\
& +\frac{1}{2} \phi_{\pi}^{2}\left(\kappa_{i, 1} D_{i, 1}+(\theta-1) \kappa_{1} A_{1}\right)^{2} \\
& +\frac{1}{2} \kappa_{1} \sigma_{w}^{2} A_{4}(\theta-1)\left(2 \kappa_{i, 1} D_{i, 4}+\kappa_{1}(\theta-1)\left(A_{4}+4(1-v) \varphi_{0} A_{5}\right)\right) \\
& +\sigma_{w}^{2}\left(2 \varphi_{0}^{2}\left(1-v^{2}\right)+\sigma_{w}^{2}\right)\left(\kappa_{i, 1} D_{i, 5}+\kappa_{1} A_{5}(\theta-1)\right)^{2} \\
& +\kappa_{i, 1} \varphi_{0}\left(\kappa_{i, 1} D_{i, 2}+(\theta-1) \kappa_{1} A_{2}\right)\left(\phi_{\pi} D_{i, 1}+D_{i, 3}\right) \\
& +\kappa_{1}(\theta-1) \varphi_{0}\left(\kappa_{i, 1} D_{i, 2}+(\theta-1) \kappa_{1} A_{2}\right)\left(A_{3}+\phi_{\pi} A_{1}\right) \\
& +\frac{1}{2} \kappa_{i, 1}\left(\kappa_{i, 1} D_{i, 3}+(\theta-1) \kappa_{1} A_{3}\right)\left(2 \phi_{\pi} D_{i, 1}+D_{i, 3}\right) \\
& +\frac{1}{2} \kappa_{1}(\theta-1)\left(\kappa_{i, 1} D_{i, 3}+(\theta-1) \kappa_{1} A_{3}\right)\left(A_{3}+2 \phi_{\pi} A_{1}\right) \\
& +\frac{1}{2}\left(\varphi_{0}^{2}+\frac{\sigma_{w}^{2}}{1-v^{2}}\right) \kappa_{1}^{2}(\theta-1)^{2}\left(A_{2}^{2}+4 v^{2} \sigma_{w}^{2} A_{5}^{2}\right) \\
& +\left(\varphi_{0}^{2}+\frac{\sigma_{w}^{2}}{1-v^{2}}\right) \kappa_{i, 1} \kappa_{1}(\theta-1)\left(A_{2} D_{i, 2}+4 v^{2} \sigma_{w}^{2} A_{5} D_{i, 5}\right) \\
& +\kappa_{i, 1}^{2}\left(D_{i, 2}^{2}+4 v^{2} \sigma_{w}^{2} D_{i, 5}^{2}\right) \\
& +\frac{1}{2} \sigma_{w}^{2} \kappa_{i, 1} D_{i, 4} \kappa_{i, 1}\left(4 \varphi_{0} D_{i, 5}+D_{i, 4}\right) \\
& +\sigma_{w}^{2} \kappa_{i, 1} \sigma_{w}^{2} \kappa_{1} \varphi_{0}(\theta-1)\left(A_{4} D_{i, 5}+A_{5} D_{i, 4}\right) \\
& +2 \sigma_{w}^{2} v \kappa_{1}^{2} \varphi_{0} A_{4} A_{5}(\theta-1)^{2} .
\end{aligned}
$$

We solve for $D_{i, j}$ for $j=1,2,3,4,5$ in terms of $D_{i, 0}$ in closed form, picking the negative root in the solution of the quadratic equation for $D_{i, 5}$, as we did for the solution of the system of equations for $A_{i}$. We then solve for $D_{i, 0}$ numerically using equations (OA.25), (OA.24) and (OA.45).

\subsection{Conditional Pricing}

We first write the SDF in terms of state variables $\left(\pi_{t}-\mu_{\pi}, \varphi_{t-1} u_{t}, u_{t}, \varphi_{t}-\varphi_{0}, \varphi_{t}^{2}-E\left[\varphi_{t}^{2}\right]\right)$ and innova-

tions $\left(u_{t+1}, w_{t+1}, \frac{w_{t+1}^{2}-1}{\sqrt{2}}, \eta_{t+1}\right)$. Note that the innovations are mean zero, variance one and orthogonal to each other (even though $w_{t+1}$ and $w_{t+1}^{2}$ are not independent) since

$$
E_{t}\left[w_{t+1}\left(w_{t+1}^{2}-1\right)\right]=E_{t}\left[w_{t+1}^{3}\right]-E_{t}\left[w_{t+1}\right]=0
$$


The innovation to the SDF is

$$
\begin{aligned}
m_{t+1}-E_{t}\left[m_{t+1}\right]= & \kappa_{1}(\theta-1)\left(\phi_{\pi} A_{1}+\varphi_{t} A_{2}+A_{3}\right) u_{t+1} \\
& +\kappa_{1} \sigma_{w}(\theta-1)\left(A_{4}+2 \varphi_{0} A_{5}(1-v)+2 v A_{5} \varphi_{t}\right) w_{t+1} \\
& +\sqrt{2} \kappa_{1} \sigma_{w}^{2}(\theta-1) A_{5}\left(\frac{w_{t+1}^{2}-1}{\sqrt{2}}\right) \\
& +\left(\theta\left(1-\frac{1}{\psi}\right)-1\right) \sigma_{c} \eta_{t+1} .
\end{aligned}
$$

We can then write

$$
m_{t+1}-E_{t}\left[m_{t+1}\right]=-\lambda_{u, t} u_{t+1}-\lambda_{w, t} w_{t+1}-\lambda_{2 w}\left(\frac{w_{t+1}^{2}-1}{\sqrt{2}}\right)-\lambda_{\eta} \eta_{t+1},
$$

where the prices of risk for $\left(u_{t+1}, w_{t+1}, \frac{w_{t+1}^{2}-1}{\sqrt{2}}, \eta_{t+1}\right)$ are

$$
\begin{aligned}
\lambda_{u, t} & \equiv-\frac{\operatorname{Cov}_{t}\left(m_{t+1}, u_{t+1}\right)}{\operatorname{Var}_{t}\left(u_{t+1}\right)} \\
& =-\kappa_{1}(\theta-1)\left(\phi_{\pi} A_{1}+\varphi_{t} A_{2}+A_{3}\right) \\
& =\kappa_{1}\left(\gamma-\frac{1}{\psi}\right)\left(\frac{\rho_{c}\left(\phi_{\pi}+\kappa_{1} \xi_{\pi}\right)}{1-\rho_{\pi} \kappa_{1}}+\xi_{c} \varphi_{t}\right), \\
\lambda_{w, t} & \equiv-\frac{\operatorname{Cov}_{t}\left(m_{t+1}, w_{t+1}\right)}{\operatorname{Var}_{t}\left(w_{t+1}\right)} \\
& =-\kappa_{1} \sigma_{w}(\theta-1)\left(A_{4}+2 \varphi_{0} A_{5}(1-v)+2 v A_{5} \varphi_{t}\right), \\
\lambda_{2 w} & \equiv-\frac{\operatorname{Cov}_{t}\left(m_{t+1},\left(w_{t+1}^{2}-1\right) / \sqrt{2}\right)}{\operatorname{Var}_{t}\left(\left(w_{t+1}^{2}-1\right) / \sqrt{2}\right)} \\
& =-\sqrt{2} \kappa_{1} \sigma_{w}^{2}(\theta-1) A_{5}, \\
\lambda_{\eta} & \equiv-\frac{\operatorname{Cov}_{t}\left(m_{t+1}, \eta_{t+1}\right)}{\operatorname{Var}_{t}\left(\eta_{t+1}\right)} \\
& =\gamma \sigma_{c} .
\end{aligned}
$$

For returns of asset $i$, we have

$$
r_{i, t+1}=E_{t}\left[r_{i, t+1}\right]+\beta_{u, i t} u_{t+1}+\beta_{w, i} w_{t+1}+\beta_{2 w, i}\left(\frac{w_{t+1}^{2}-1}{\sqrt{2}}\right)+\beta_{\eta, i} \eta_{t+1},
$$

where the conditional return is

$$
\begin{aligned}
E_{t}\left[r_{i, t+1}\right]= & \kappa_{i, 0}+\mu_{i}-D_{i, 0}\left(1-\kappa_{i, 1}\right) \\
& +\left(\rho_{i}-D_{i, 1}\left(1-\kappa_{i, 1} \rho_{\pi}\right)\right)\left(\pi_{t}-\mu_{\pi}\right) \\
& -u_{t}\left(D_{i, 3}-\xi_{\pi} \kappa_{i, 1} D_{i, 1}\right)+\left(\xi_{i}-D_{i, 2}\right) \varphi_{t-1} u_{t} \\
& +\left(2 D_{i, 5} v \kappa_{i, 1} \varphi_{0}(1-v)-D_{i, 4}\left(1-v \kappa_{i, 1}\right)\right)\left(\varphi_{t}-\varphi_{0}\right) \\
& -D_{i, 5}\left(1-v^{2} \kappa_{i, 1}\right)\left(\varphi_{t}^{2}-E\left[\varphi_{t}^{2}\right]\right),
\end{aligned}
$$


and the quantities of risk are given by the betas

$$
\begin{aligned}
\beta_{u, i t} & \equiv \frac{\operatorname{Cov}_{t}\left(u_{t+1}, r_{i, t+1}\right)}{\operatorname{Var}_{t}\left(u_{t+1}\right)} \\
& =\kappa_{i, 1}\left(\phi_{\pi} D_{i, 1}+D_{i, 2} \varphi_{t}+D_{i, 3}\right) \\
& =\kappa_{i, 1}\left(\left(\frac{\rho_{i}}{\rho_{c}}-\frac{1}{\psi}\right) \frac{\left(\phi_{\pi}+\xi_{\pi} \kappa_{i, 1}\right) \rho_{c}}{1-\rho_{\pi} \kappa_{i, 1}}+\left(\frac{\xi_{i}}{\xi_{c}}-\frac{1}{\psi}\right) \xi_{c} \varphi_{t}\right) \\
\beta_{w, i t} & \equiv \frac{\operatorname{Cov}_{t}\left(w_{t+1}, r_{i, t+1}\right)}{\operatorname{Var}_{t}\left(w_{t+1}\right)} \\
& =\kappa_{i, 1} \sigma_{w}\left(D_{i, 4}+2 \varphi_{0} D_{i, 5}(1-v)+2 v D_{i, 5} \varphi_{t}\right), \\
\beta_{2 w, i} & \equiv \frac{\operatorname{Cov}_{t}\left(\left(w_{t+1}^{2}-1\right) / \sqrt{2}, r_{i, t+1}\right)}{\operatorname{Var}_{t}\left(\left(w_{t+1}^{2}-1\right) / \sqrt{2}\right)} \\
& =\sqrt{2} \kappa_{i, 1} \sigma_{w}^{2} D_{i, 5}, \\
\beta_{\eta, i} & \equiv \frac{\operatorname{Cov}_{t}\left(\eta_{t+1}, r_{i, t+1}\right)}{\operatorname{Var}_{t}\left(\eta_{t+1}\right)} \\
& =\sigma_{i} .
\end{aligned}
$$

Expected excess returns for asset $i$ can then be written

$$
\begin{aligned}
-\operatorname{Cov}_{t}\left(m_{t+1}, r_{i, t+1}\right) & =-E_{t}\left[\left(m_{t+1}-E_{t}\left[m_{t+1}\right]\right)\left(r_{i, t+1}-E_{t}\left[r_{i, t+1}\right]\right)\right] \\
& =\lambda_{u, t} \beta_{u, i t}+\lambda_{w, t} \beta_{w, i t}+\lambda_{2 w} \beta_{2 w, i}+\lambda_{\eta} \beta_{\eta, i} .
\end{aligned}
$$

Of course, we can always set $i=c$ and study the consumption portfolio:

$$
r_{c, t+1}=E_{t}\left[r_{c, t+1}\right]+\beta_{u, c t} u_{t+1}+\beta_{w, c t} w_{t+1}+\beta_{2 w, c}\left(\frac{w_{t+1}^{2}-1}{\sqrt{2}}\right)+\beta_{\eta, c} \eta_{t+1},
$$

where

$$
\begin{aligned}
E_{t}\left[r_{c, t+1}\right]= & \kappa_{0}+\mu_{c}-A_{0}\left(1-\kappa_{1}\right) \\
& +\left(\rho_{c}-A_{1}\left(1-\kappa_{1} \rho_{\pi}\right)\right)\left(\pi_{t}-\mu_{\pi}\right) \\
& +\left(\xi_{\pi} \kappa_{1} A_{1}-A_{3}\right) u_{t} \\
& +\left(\xi_{c}-A_{2}\right) \varphi_{t-1} u_{t} \\
& +\left(2 v \kappa_{1} \varphi_{0}(1-v) A_{5}-\left(1-v \kappa_{1}\right) A_{4}\right)\left(\varphi_{t}-\varphi_{0}\right) \\
& -A_{5}\left(1-v^{2} \kappa_{1}\right)\left(\varphi_{t}^{2}-E\left[\varphi_{t}^{2}\right]\right),
\end{aligned}
$$


and where the quantities of risk are given by the betas

$$
\begin{aligned}
\beta_{u, c t} & =\frac{\operatorname{Cov}_{t}\left(u_{t+1}, r_{c, t+1}\right)}{\operatorname{Var}_{t}\left(u_{t+1}\right)} \\
& =\kappa_{1}\left(\phi_{\pi} A_{1}+A_{2} \varphi_{t}+A_{3}\right), \\
\beta_{w, c t} & =\frac{\operatorname{Cov}_{t}\left(w_{t+1}, r_{c, t+1}\right)}{\operatorname{Var}_{t}\left(w_{t+1}\right)} \\
& =\kappa_{1} \sigma_{w}\left(\left(A_{4}+2 \varphi_{0} A_{5}(1-v)\right)+2 v A_{5} \varphi_{t}\right), \\
\beta_{2 w, c} & =\frac{\operatorname{Cov}_{t}\left(\left(w_{t+1}^{2}-1\right) / \sqrt{2}, r_{c, t+1}\right)}{\operatorname{Var}_{t}\left(\left(w_{t+1}^{2}-1\right) / \sqrt{2}\right)} \\
& =\sqrt{2} \kappa_{1} \sigma_{w}^{2} A_{5}, \\
\beta_{\eta, c} & =\frac{\operatorname{Cov}_{t}\left(\eta_{t+1}, r_{c, t+1}\right)}{\operatorname{Var}_{t}\left(\eta_{t+1}\right)} \\
& =\sigma_{c} .
\end{aligned}
$$

Expected excess returns for asset $c$ can then be written

$$
\begin{aligned}
-\operatorname{Cov}_{t}\left(m_{t+1}, r_{c, t+1}\right) & =-E_{t}\left[\left(m_{t+1}-E_{t}\left[m_{t+1}\right]\right)\left(r_{c, t+1}-E_{t}\left[r_{c, t+1}\right]\right)\right] \\
& =\lambda_{u, t} \beta_{u, c t}+\lambda_{w, t} \beta_{w, c t}+\lambda_{2 w} \beta_{2 w, c}+\lambda_{\eta} \beta_{\eta, c} .
\end{aligned}
$$

Inflation betas and the price of risk with respect to the shock $u_{\Pi, t+1} \equiv \phi_{\pi} u_{t+1}$ are

$$
\begin{aligned}
\beta_{\Pi, i, t} & =\phi_{\pi} \beta_{u, i t}, \\
\lambda_{\Pi, t} & =\frac{\lambda_{u, t}}{\phi_{\pi}} .
\end{aligned}
$$


2.6 Derivation of equations (20) and (21)

First, we compute innovations for $\pi_{t+2+j}, \varphi_{t+2+j}$ and $\varphi_{t+2+j}^{2}$ :

$$
\begin{aligned}
& \pi_{t+2+j}=\mu_{\pi}+\rho_{\pi}\left(\pi_{t+1+j}-\mu_{\pi}\right)+\phi_{\pi} u_{t+2+j}+\xi_{\pi} u_{t+1+j} \\
& E_{t+1+j}\left[\pi_{t+2+j}\right]=\mu_{\pi}+\rho_{\pi}\left(\pi_{t+1+j}-\mu_{\pi}\right)+\xi_{\pi} u_{t+1+j}, \\
& \pi_{t+2+j}-E_{t+1+j}\left[\pi_{t+2+j}\right]=\phi_{\pi} u_{t+2+j}, \\
& \varphi_{t+2+j}=\varphi_{0}+v\left(\varphi_{t+1+j}-\varphi_{0}\right)+\sigma_{w} w_{t+2+j}, \\
& E_{t+1+j}\left[\varphi_{t+2+j}\right]=\varphi_{0}+v\left(\varphi_{t+1+j}-\varphi_{0}\right), \\
& \varphi_{t+2+j}-E_{t+1+j}\left[\varphi_{t+2+j}\right]=\sigma_{w} w_{t+2+j} \text {, } \\
& \varphi_{t+2+j}^{2}=\left(\varphi_{0}+v\left(\varphi_{t+1+j}-\varphi_{0}\right)\right)^{2}+\sigma_{w}^{2} w_{t+2+j}^{2} \\
& +2\left(\varphi_{0}+v\left(\varphi_{t+1+j}-\varphi_{0}\right)\right) \sigma_{w} w_{t+2+j}, \\
& E_{t+1+j}\left[\varphi_{t+2+j}^{2}\right]=\left(\varphi_{0}+v\left(\varphi_{t+1+j}-\varphi_{0}\right)\right)^{2}+\sigma_{w}^{2}, \\
& \varphi_{t+2+j}^{2}-E_{t+1+j}\left[\varphi_{t+2+j}^{2}\right]=\sqrt{2} \sigma_{w}^{2}\left(\frac{w_{t+2+j}^{2}-1}{\sqrt{2}}\right) \\
& +2\left(\varphi_{0}+v\left(\varphi_{t+1+j}-\varphi_{0}\right)\right) \sigma_{w} w_{t+2+j} \text {. }
\end{aligned}
$$


We use these innovations to compute innovations in $w c_{t+2+j}$

$$
\begin{aligned}
w c_{t+2+j}= & A_{0}+A_{1}\left(\pi_{t+2+j}-\mu_{\pi}\right)+A_{2} \varphi_{t+1+j} u_{t+2+j}+A_{3} u_{t+2+j} \\
& +A_{4}\left(\varphi_{t+2+j}-\varphi_{0}\right)+A_{5}\left(\varphi_{t+2+j}^{2}-E\left[\varphi_{t}^{2}\right]\right) \\
E_{t+1+j}\left[w c_{t+2+j}\right]= & A_{0}+A_{1}\left(E_{t+1+j}\left[\pi_{t+2+j}\right]-\mu_{\pi}\right)+A_{2} E_{t+1+j}\left[\varphi_{t+1+j} u_{t+2+j}\right] \\
& +A_{3} E_{t+1+j}\left[u_{t+2+j}\right]+A_{4}\left(E_{t+1+j}\left[\varphi_{t+2+j}\right]-\varphi_{0}\right) \\
& +A_{5}\left(E_{t+1+j}\left[\varphi_{t+2+j}^{2}\right]-E\left[\varphi_{t}^{2}\right]\right) \\
w c_{t+2+j}-E_{t+1+j}\left[w c_{t+2+j}\right]= & A_{1}\left(\pi_{t+2+j}-E_{t+1+j}\left[\pi_{t+2+j}\right]\right)+A_{2} \varphi_{t+1+j} u_{t+2+j} \\
& +A_{3} u_{t+2+j}+A_{4}\left(\varphi_{t+2+j}-E_{t+1+j}\left[\varphi_{t+2+j}\right]\right) \\
& +A_{5}\left(\varphi_{t+2+j}^{2}-E_{t+1+j}\left[\varphi_{t+2+j}^{2}\right]\right) \\
= & A_{1}\left(\pi_{t+2+j}-E_{t+1+j}\left[\pi_{t+2+j}\right]\right)+A_{2} \varphi_{t+1+j} u_{t+2+j} \\
& +A_{3} u_{t+2+j}+A_{4}\left(\varphi_{t+2+j}-E_{t+1+j}\left[\varphi_{t+2+j}\right]\right) \\
& +A_{5}\left(\varphi_{t+2+j}^{2}-E_{t+1+j}\left[\varphi_{t+2+j}^{2}\right]\right) \\
= & \left(A_{1} \phi_{\pi}+A_{2} \varphi_{t+1+j}+A_{3}\right) u_{t+2+j} \\
& +\left(A_{4}+2 A_{5}\left(\varphi_{0}+v\left(\varphi_{t+1+j}-\varphi_{0}\right)\right)\right) \sigma_{w} w_{t+2+j} \\
& +A_{5} \sqrt{2} \sigma_{w}^{2}\left(\frac{w_{t+2+j}^{2}-1}{\sqrt{2}}\right)
\end{aligned}
$$

Innovations in consumption $\Delta c_{t+2+j}$ are

$$
\begin{aligned}
\Delta c_{t+2+j} & =\mu_{c}+\rho_{c}\left(\pi_{t+1+j}-\mu_{\pi}\right)+\sigma_{c} \eta_{t+2+j}+\xi_{c} \varphi_{t+j} u_{t+1+j}, \\
E_{t+1+j}\left[\Delta c_{t+2+j}\right] & =\mu_{c}+\rho_{c}\left(\pi_{t+1+j}-\mu_{\pi}\right)+\xi_{c} \varphi_{t+j} u_{t+1+j}, \\
\Delta c_{t+2+j}-E_{t+1+j}\left[\Delta c_{t+2+j}\right] & =\sigma_{c} \eta_{t+2+j} .
\end{aligned}
$$

Since the shocks $u_{t+2+j}, w_{t+2+j},\left(w_{t+2+j}^{2}-1\right) / \sqrt{2}$ are independent of $\eta_{t+2+j}$, equations (OA.60) and (OA.61) imply

$$
\operatorname{Cov}_{t+1+j}\left(\Delta c_{t+2+j}, w c_{t+2+j}\right)=0 .
$$

Eq. (OA.26) gives

$$
\begin{aligned}
m_{t+2+j}+r_{c, t+2+j} & =\theta\left(\log \delta+\kappa_{0}\right)+\theta\left(1-\frac{1}{\psi}\right) \Delta c_{t+2+j}+\theta\left(\kappa_{1} w c_{t+2+j}-w c_{t+1+j}\right) \\
& =\theta\left(\log \delta+\kappa_{0}\right)-\theta w c_{t+1+j}+\theta\left(1-\frac{1}{\psi}\right) \Delta c_{t+2+j}+\theta \kappa_{1} w c_{t+2+j} .
\end{aligned}
$$


Eqs. (OA.61) and (OA.62) then imply

$$
\begin{aligned}
\operatorname{Var}_{t+1+j}\left(m_{t+2+j}+r_{c, t+2+j}\right)= & \operatorname{Var}_{t+1+j}\left(\theta\left(1-\frac{1}{\psi}\right) \Delta c_{t+2+j}+\theta \kappa_{1} w c_{t+2+j}\right) \\
= & \theta^{2}\left(1-\frac{1}{\psi}\right)^{2} \operatorname{Var}_{t+1+j}\left(\Delta c_{t+2+j}\right) \\
& +\left(\theta \kappa_{1}\right)^{2} \operatorname{Var}_{t+1+j}\left(w c_{t+2+j}\right) \\
& +2 \theta^{2} \kappa_{1}\left(1-\frac{1}{\psi}\right) \operatorname{Cov}_{t+1+j}\left(\Delta c_{t+2+j}, w c_{t+2+j}\right) \\
= & \theta^{2}\left(1-\frac{1}{\psi}\right)^{2} \sigma_{c}^{2}+\left(\theta \kappa_{1}\right)^{2} \operatorname{Var}_{t+1+j}\left(w c_{t+2+j}\right) .
\end{aligned}
$$

The innovations in $\operatorname{Var}_{t+1+j}\left(m_{t+2+j}+r_{c, t+2+j}\right)$ are

$$
\left(E_{t+1}-E_{t}\right) \operatorname{Var}_{t+1+j}\left(m_{t+2+j}+r_{c, t+2+j}\right)=\left(\theta \kappa_{1}\right)^{2}\left(E_{t+1}-E_{t}\right) \operatorname{Var}_{t+1+j}\left(w c_{t+2+j}\right) .
$$

Plug (OA.63) evaluated at $j=1$ into the first term of the Euler equation (OA.27) evaluated at $t+2$ to get

$$
\begin{aligned}
0= & E_{t+1}\left[m_{t+2}+r_{c, t+2}\right]+\frac{1}{2} \operatorname{Var}_{t+1}\left[m_{t+2}+r_{c, t+2}\right], \\
0= & E_{t+1}\left[\theta\left(\log \delta+\kappa_{0}\right)+\theta\left(1-\frac{1}{\psi}\right) \Delta c_{t+2}+\theta\left(\kappa_{1} w c_{t+2}-w c_{t+1}\right)\right] \\
& +\frac{1}{2} \operatorname{Var}_{t+1}\left[m_{t+2}+r_{c, t+2}\right] .
\end{aligned}
$$

Solving for $w c_{t+1}$ and iterating forward

$$
\begin{aligned}
w c_{t+1}= & E_{t+1}\left[\log \delta+\kappa_{0}+\left(1-\frac{1}{\psi}\right) \Delta c_{t+2}+\kappa_{1} w c_{t+2}\right]+\frac{1}{2 \theta} \operatorname{Var}_{t+1}\left[m_{t+2}+r_{c, t+2}\right] \\
= & \log \delta+\kappa_{0}+\left(1-\frac{1}{\psi}\right) E_{t+1}\left[\Delta c_{t+2}\right]+\frac{1}{2 \theta} \operatorname{Var}_{t+1}\left[m_{t+2}+r_{c, t+2}\right]+\kappa_{1} E_{t+1}\left[w c_{t+2}\right] \\
= & \log \delta+\kappa_{0}+\left(1-\frac{1}{\psi}\right) E_{t+1}\left[\Delta c_{t+2}\right]+\frac{1}{2 \theta} \operatorname{Var}_{t+1}\left[m_{t+2}+r_{c, t+2}\right] \\
& +\kappa_{1} E_{t+1}\left[\log \delta+\kappa_{0}+\left(1-\frac{1}{\psi}\right) E_{t+2}\left[\Delta c_{t+3}\right]+\frac{1}{2 \theta} \operatorname{Var}_{t+2}\left[m_{t+3}+r_{c, t+3}\right]+\kappa_{1} E_{t+2}\left[w c_{t+3}\right]\right] \\
= & \left(1+\kappa_{1}\right)\left(\log \delta+\kappa_{0}\right) \\
& +\left(1-\frac{1}{\psi}\right)\left[E_{t+1}\left[\Delta c_{t+2}\right]+\kappa_{1} E_{t+2}\left[\Delta c_{t+3}\right]\right] \\
& +\frac{1}{2 \theta} E_{t+1}\left[\operatorname{Var}_{t+1}\left[m_{t+2}+r_{c, t+2}\right]+\kappa_{1} \operatorname{Var}_{t+2}\left[m_{t+3}+r_{c, t+3}\right]\right] \\
& +\kappa_{1}^{2} E_{t+1}\left[w c_{t+3}\right] \\
= & \ldots \\
= & \left(\log \delta+\kappa_{0}\right) \sum_{j=0}^{\infty} \kappa_{1}^{j}+\left(1-\frac{1}{\psi}\right) E_{t+1} \sum_{j=0}^{\infty} \kappa_{1}^{j} E_{t+1+j}\left[\Delta c_{t+2+j}\right] \\
& +\frac{1}{2 \theta} E_{t+1} \sum_{j=0}^{\infty} \kappa_{1}^{j} \operatorname{Var}_{t+1+j}\left[m_{t+2+j}+r_{c, t+2+j}\right]+\lim _{j \rightarrow \infty} \kappa_{1}^{j} E_{t+1+j} w c_{t+2+j} .
\end{aligned}
$$


Assuming the that the bubble term vanishes and applying $\left(E_{t+1}-E_{t}\right)$ to both sides gives

$$
\begin{aligned}
\left(E_{t+1}-E_{t}\right) w c_{t+1}= & \left(1-\frac{1}{\psi}\right)\left(E_{t+1}-E_{t}\right) \sum_{j=0}^{\infty} \kappa_{1}^{j} E_{t+1+j}\left[\Delta c_{t+2+j}\right] \\
& +\frac{1}{2 \theta}\left(E_{t+1}-E_{t}\right) \sum_{j=0}^{\infty} \kappa_{1}^{j} \operatorname{Var}_{t+1+j}\left[m_{t+2+j}+r_{c, t+2+j}\right] .
\end{aligned}
$$

Using (OA.64) in (OA.66) gives

$$
\begin{aligned}
\left(E_{t+1}-E_{t}\right) w c_{t+1}= & \left(1-\frac{1}{\psi}\right)\left(E_{t+1}-E_{t}\right) \sum_{j=0}^{\infty} \kappa_{1}^{j} E_{t+1+j}\left[\Delta c_{t+2+j}\right] \\
& +\frac{1}{2 \theta}\left(E_{t+1}-E_{t}\right) \sum_{j=0}^{\infty} \kappa_{1}^{j} \operatorname{Var}_{t+1+j}\left[m_{t+2+j}+r_{c, t+2+j}\right] \\
= & \left(1-\frac{1}{\psi}\right)\left(E_{t+1}-E_{t}\right) \sum_{j=0}^{\infty} \kappa_{1}^{j} E_{t+1+j}\left[\Delta c_{t+2+j}\right] \\
& +\frac{\theta \kappa_{1}^{2}}{2}\left(E_{t+1}-E_{t}\right) \sum_{j=0}^{\infty} \kappa_{1}^{j} \operatorname{Var}_{t+1+j}\left(w c_{t+2+j}\right) .
\end{aligned}
$$

Plugging (OA.20) into (OA.9) and using (OA.10) gives

$$
\begin{aligned}
m_{t+1} & =\theta \log \delta-\frac{\theta}{\psi} \Delta c_{t+1}-(1-\theta) r_{c, t+1} \\
& =\theta \log \delta+(\theta-1) \kappa_{0}-(\theta-1) w c_{t}-\gamma \Delta c_{t+1}-(1-\theta) \kappa_{1} w c_{t+1} .
\end{aligned}
$$

Applying $\left(E_{t+1}-E_{t}\right)$ to both sides and using (OA.67) gives equations (20)-(21)

$$
\begin{aligned}
m_{t+1}-E_{t} m_{t+1}= & -\gamma\left(E_{t+1}-E_{t}\right) \Delta c_{t+1}-(1-\theta) \kappa_{1}\left(E_{t+1}-E_{t}\right) w c_{t+1} \\
= & -\gamma\left(E_{t+1}-E_{t}\right) \Delta c_{t+1} \\
& -(1-\theta) \kappa_{1}\left(\left(1-\frac{1}{\psi}\right)\left(E_{t+1}-E_{t}\right) \sum_{j=0}^{\infty} \kappa_{1}^{j} E_{t+1+j}\left[\Delta c_{t+2+j}\right]\right. \\
& \left.+\frac{\theta \kappa_{1}^{2}}{2}\left(E_{t+1}-E_{t}\right) \sum_{j=0}^{\infty} \kappa_{1}^{j} \operatorname{Var}_{t+1+j}\left(w c_{t+2+j}\right)\right) \\
= & -\gamma N_{C, t+1}-(1-\theta) \kappa_{1}\left(\left(1-\frac{1}{\psi}\right) N_{P A T H, t+1}+\frac{\theta \kappa_{1}^{2}}{2} N_{R I S K, t+1}\right),
\end{aligned}
$$

where we define

$$
\begin{aligned}
N_{C, t+1} & \equiv\left(E_{t+1}-E_{t}\right) \Delta c_{t+1}, \\
N_{P A T H, t+1} & \equiv\left(E_{t+1}-E_{t}\right) \sum_{j=0}^{\infty} \kappa_{1}^{j} E_{t+1+j}\left[\Delta c_{t+2+j}\right], \\
N_{R I S K, t+1} & \equiv\left(E_{t+1}-E_{t}\right) \sum_{j=0}^{\infty} \kappa_{1}^{j} \operatorname{Var}_{t+1+j}\left(w c_{t+2+j}\right) .
\end{aligned}
$$




\subsection{Shocks Driving $N_{C, t+1}, N_{P A T H, t+1}$, and $N_{R I S K, t+1}$}

Using equation (14), news to $N_{C, t+1}$ depends only on the shock $\eta_{t+1}$, such that

$$
-\gamma N_{C, t+1}=-\gamma \sigma_{c} \eta_{t+1}
$$

$N_{P A T H, t+1}$ in equation (22) can be obtained by direct computation using equation (14) and the law of iterated expectations:

$$
\begin{aligned}
N_{P A T H, t+1} & =\left(E_{t+1}-E_{t}\right) \sum_{j=0}^{\infty} \kappa_{1}^{j} E_{t+1+j}\left[\Delta c_{t+2+j}\right] \\
& =\left(E_{t+1}-E_{t}\right) \sum_{j=0}^{\infty} \kappa_{1}^{j} \Delta c_{t+2+j} \\
& =\left(E_{t+1}-E_{t}\right) \Delta c_{t+2}+\left(E_{t+1}-E_{t}\right) \sum_{j=1}^{\infty} \kappa_{1}^{j} \Delta c_{t+2+j} \\
& =\left(\phi_{\pi} \rho_{c}+\xi_{c} \varphi_{t}\right) u_{t+1}+\sum_{j=1}^{\infty} \kappa_{1}^{j} \rho_{\pi}^{j-1} \rho_{c}\left(\xi_{\pi}+\rho_{\pi} \phi_{\pi}\right) u_{t+1} \\
& =\left(\frac{\phi_{\pi} \rho_{c}}{1-\rho_{\pi} \kappa_{1}}+\xi_{c} \varphi_{t}+\frac{\rho_{c} \kappa_{1} \xi_{\pi}}{1-\rho_{\pi} \kappa_{1}}\right) u_{t+1} .
\end{aligned}
$$

We now show that $N_{R I S K, t+1}$ depends only on the nominal-real covariance shock $w_{t+1}$. Eqs. (21) and (OA.46) give

$$
\begin{aligned}
m_{t+1}-E_{t}\left[m_{t+1}\right]= & -\gamma N_{C, t+1}-(1-\theta) \kappa_{1}\left(\left(1-\frac{1}{\psi}\right) N_{P A T H, t+1}+\frac{\theta \kappa_{1}^{2}}{2} N_{R I S K, t+1}\right) \\
= & \kappa_{1}(\theta-1)\left(\phi_{\pi} A_{1}+\varphi_{t} A_{2}+A_{3}\right) u_{t+1} \\
& +\left(\theta\left(1-\frac{1}{\psi}\right)-1\right) \sigma_{c} \eta_{t+1} \\
& +\kappa_{1} \sigma_{w}(\theta-1)\left(A_{4}+2 \varphi_{0} A_{5}(1-v)+2 v A_{5} \varphi_{t}\right) w_{t+1} \\
& +\sqrt{2} \kappa_{1} \sigma_{w}^{2}(\theta-1) A_{5}\left(\frac{w_{t+1}^{2}-1}{\sqrt{2}}\right) .
\end{aligned}
$$

Solving for $\frac{\kappa_{1}^{3}}{2} \theta(\theta-1) N_{R I S K, t+1}$ gives

$$
\begin{aligned}
+\frac{\kappa_{1}^{3}}{2} \theta(\theta-1) N_{R I S K, t+1}= & \kappa_{1}(\theta-1)\left(\phi_{\pi} A_{1}+\varphi_{t} A_{2}+A_{3}\right) u_{t+1} \\
& -(\theta-1) \kappa_{1}\left(1-\frac{1}{\psi}\right) N_{P A T H, t+1} \\
& +\left(\theta\left(1-\frac{1}{\psi}\right)-1\right) \sigma_{c} \eta_{t+1}+\gamma N_{C, t+1} \\
& +\kappa_{1} \sigma_{w}(\theta-1)\left(A_{4}+2 \varphi_{0} A_{5}(1-v)+2 v A_{5} \varphi_{t}\right) w_{t+1} \\
& +\sqrt{2} \kappa_{1} \sigma_{w}^{2}(\theta-1) A_{5}\left(\frac{w_{t+1}^{2}-1}{\sqrt{2}}\right) .
\end{aligned}
$$


Using (OA.34)-(OA.36), (OA.68) and (OA.69), the last equation becomes

$$
\begin{aligned}
\frac{\kappa_{1}^{3}}{2} \theta(\theta-1) N_{R I S K, t+1}= & \kappa_{1} \sigma_{w}(\theta-1)\left(A_{4}+2 \varphi_{0} A_{5}(1-v)+2 v A_{5} \varphi_{t}\right) w_{t+1} \\
& +\sqrt{2} \kappa_{1} \sigma_{w}^{2}(\theta-1) A_{5}\left(\frac{w_{t+1}^{2}-1}{\sqrt{2}}\right)
\end{aligned}
$$

which shows $N_{R I S K, t+1}$ depends on the shock $w_{t+1}$ but neither on $\eta_{t+1}$ nor $u_{t+1}$. Using equations (OA.34)(OA.38) and (OA.47)-(OA.50), equations (OA.68), (OA.69) and (OA.70) can be written as

$$
\begin{aligned}
\gamma N_{C, t+1} & =\lambda_{\eta} \eta_{t+1}, \\
\kappa_{1}(1-\theta)\left(1-\frac{1}{\psi}\right) N_{P A T H, t+1} & =\lambda_{u, t} u_{t+1}, \\
\frac{\kappa_{1}^{3}}{2} \theta(\theta-1) N_{R I S K, t+1} & =-\lambda_{w, t} w_{t+1}-\lambda_{2 w}\left(\frac{w_{t+1}^{2}-1}{\sqrt{2}}\right) .
\end{aligned}
$$

\subsection{Derivation of equation (25)}

Using equation (OA.21) in equation (OA.27) and solving for $p d_{i, t+1}$ gives

$$
p d_{i, t+1}=E_{t+1}\left[m_{t+2}+\kappa_{i, 0}+\Delta d_{i, t+2}+\kappa_{i, 1} p d_{i, t+2}\right]+\frac{1}{2} \operatorname{Var}_{t+1}\left[m_{t+2}+\kappa_{i, 1} p d_{i, t+2}+\Delta d_{i, t+2}\right] .
$$

Iterating forward, we get

$$
\begin{aligned}
p d_{i, t+1}= & E_{t+1}\left[m_{t+2}+\kappa_{i, 0}+\Delta d_{i, t+2}\right]+\frac{1}{2} \operatorname{Var}_{t+1}\left[m_{t+2}+\kappa_{i, 1} p d_{i, t+2}+\Delta d_{i, t+2}\right] \\
& +\kappa_{i, 1} E_{t+1}\left[p d_{i, t+2}\right] \\
= & E_{t+1}\left[m_{t+2}+\kappa_{i, 0}+\Delta d_{i, t+2}\right]+\frac{1}{2} \operatorname{Var}_{t+1}\left[m_{t+2}+\kappa_{i, 1} p d_{i, t+2}+\Delta d_{i, t+2}\right] \\
& +\kappa_{i, 1} E_{t+1}\left[E_{t+2}\left[m_{t+3}+\kappa_{i, 0}+\Delta d_{i, t+3}\right]+\frac{1}{2} \operatorname{Var}_{t+2}\left[m_{t+3}+\kappa_{i, 1} p d_{i, t+3}+\Delta d_{i, t+3}\right]\right. \\
& \left.+\kappa_{i, 1} E_{t+2}\left[p d_{i, t+3}\right]\right] \\
= & E_{t+1}\left[m_{t+2}+\kappa_{i, 0}+\Delta d_{i, t+2}\right]+\kappa_{i, 1} E_{t+1} E_{t+2}\left[m_{t+3}+\kappa_{i, 0}+\Delta d_{i, t+3}\right] \\
& +\frac{1}{2} \operatorname{Var}_{t+1}\left[m_{t+2}+\kappa_{i, 1} p d_{i, t+2}+\Delta d_{i, t+2}\right]+\frac{1}{2} \kappa_{i, 1} E_{t+1} \operatorname{Var}_{t+2}\left[m_{t+3}+\kappa_{i, 1} p d_{i, t+3}+\Delta d_{i, t+3}\right] \\
& +\kappa_{i, 1} E_{t+1}\left[p d_{i, t+3}\right] \\
= & \ldots \\
= & E_{t+1} \sum_{q=0}^{\infty} \kappa_{i, 1}^{q} E_{t+1+q}\left[m_{t+2+q}+\kappa_{i, 0}+\Delta d_{i, t+2+q}\right] \\
& +\frac{1}{2} E_{t+1} \sum_{q=0}^{\infty} \kappa_{i, 1}^{q} \operatorname{Var}_{t+1+q}\left[m_{t+2+q}+\kappa_{i, 1} p d_{i, t+2+q}+\Delta d_{i, t+2+q}\right] \\
& +\lim _{q \rightarrow \infty} \kappa_{i, 1}^{q} E_{t+1}\left[p d_{i, t+2+q}\right] .
\end{aligned}
$$


Applying $\left(E_{t+1}-E_{t}\right)$ to both sides and assuming that the bubble term is zero

$$
\begin{aligned}
\left(E_{t+1}-E_{t}\right) p d_{i, t+1}= & \left(E_{t+1}-E_{t}\right) \sum_{q=0}^{\infty} \kappa_{i, 1}^{q} E_{t+1+q}\left[m_{t+2+q}\right] \\
& +\frac{1}{2}\left(E_{t+1}-E_{t}\right) \sum_{q=0}^{\infty} \kappa_{i, 1}^{q} \operatorname{Var}_{t+1+q}\left[m_{t+2+q}\right] \\
& +\left(E_{t+1}-E_{t}\right) \sum_{q=0}^{\infty} \kappa_{i, 1}^{q} E_{t+1+q}\left[\Delta d_{i, t+2+q}\right] \\
& +\frac{\kappa_{i, 1}^{2}}{2}\left(E_{t+1}-E_{t}\right) \sum_{q=0}^{\infty} \kappa_{i, 1}^{q} \operatorname{Var}_{t+1+q}\left[p d_{i, t+2+q}\right] \\
& +\frac{1}{2}\left(E_{t+1}-E_{t}\right) \sum_{q=0}^{\infty} \kappa_{i, 1}^{q} \operatorname{Var}_{t+1+q}\left[\Delta d_{i, t+2+q}\right] \\
& +\kappa_{i, 1}\left(E_{t+1}-E_{t}\right) \sum_{q=0}^{\infty} \kappa_{i, 1}^{q} \operatorname{Cov}_{t+1+q}\left[m_{t+2+q}, p d_{i, t+2+q}\right] \\
& +\kappa_{i, 1}\left(E_{t+1}-E_{t}\right) \sum_{q=0}^{\infty} \kappa_{i, 1}^{q} \operatorname{Cov}_{t+1+q}\left[p d_{i, t+2+q}, \Delta d_{i, t+2+q}\right] .
\end{aligned}
$$

Applying $\left(E_{t+1}-E_{t}\right)$ to both sides of equation (OA.21) and using equation (OA.75) gives equation (25)

$$
\begin{aligned}
\left(E_{t+1}-E_{t}\right) r_{i, t+1} & =\kappa_{i, 1}\left(E_{t+1}-E_{t}\right) p d_{i, t+1}+\left(E_{t+1}-E_{t}\right) \Delta d_{i, t+1} \\
& =\kappa_{i, 1}\left(N_{P A T H, t+1}^{i}+N_{m, t+1}^{i}+\frac{1}{2} N_{R I S K, t+1}^{i}\right)+N_{D, t+1}^{i}
\end{aligned}
$$


where we define

$$
\begin{aligned}
N_{P A T H, t+1}^{i} \equiv & \left(E_{t+1}-E_{t}\right) \sum_{q=0}^{\infty} \kappa_{i, 1}^{q} E_{t+1+q}\left[\Delta d_{i, t+2+q}\right] \\
N_{m, t+1}^{i} \equiv & \left(E_{t+1}-E_{t}\right) \sum_{q=0}^{\infty} \kappa_{i, 1}^{q} E_{t+1+q}\left[m_{t+2+q}\right] \\
N_{R I S K, t+1}^{i} \equiv & \left(E_{t+1}-E_{t}\right) \sum_{q=0}^{\infty} \kappa_{i, 1}^{q} \operatorname{Var}_{t+1+q}\left[m_{t+2+q}+\kappa_{i, 1} p d_{i, t+1}+\Delta d_{i, t+1}\right] \\
= & \frac{1}{2}\left(E_{t+1}-E_{t}\right) \sum_{q=0}^{\infty} \kappa_{i, 1}^{q} \operatorname{Var}_{t+1+q}\left[m_{t+2+q}\right] \\
& +\frac{\kappa_{i, 1}^{2}}{2}\left(E_{t+1}-E_{t}\right) \sum_{q=0}^{\infty} \kappa_{i, 1}^{q} \operatorname{Var}_{t+1+q}\left[p d_{i, t+2+q}\right] \\
& +\frac{1}{2}\left(E_{t+1}-E_{t}\right) \sum_{q=0}^{\infty} \kappa_{i, 1}^{q} \operatorname{Var}_{t+1+q}\left[\Delta d_{i, t+2+q}\right] \\
& +\kappa_{i, 1}\left(E_{t+1}-E_{t}\right) \sum_{q=0}^{\infty} \kappa_{i, 1}^{q} \operatorname{Cov}_{t+1+q}\left[m_{t+2+q}, p d_{i, t+2+q}\right] \\
& +\kappa_{i, 1}\left(E_{t+1}-E_{t}\right) \sum_{q=0}^{\infty} \kappa_{i, 1}^{q} \operatorname{Cov}_{t+1+q}\left[p d_{i, t+2+q}, \Delta d_{i, t+2+q}\right] \\
& +\kappa_{i, 1}\left(E_{t+1}-E_{t}\right) \sum_{q=0}^{\infty} \kappa_{i, 1}^{q} \operatorname{Cov}_{t+1+q}\left[m_{t+2+q}, \Delta d_{i, t+2+q}\right] \\
\equiv & \left(E_{t+1}-E_{t}\right) \Delta d_{i, t+1} .
\end{aligned}
$$

2.9 Shocks Driving $N_{P A T H, t+1}^{i}, N_{m, t+1}^{i}, N_{R I S K, t+1}^{i}$ and $N_{D, t+1}^{i}$

$N_{P A T H, t+1}^{i}$ can be computed directly from equations (OA.11)-(OA.14):

$$
\begin{aligned}
N_{P A T H, t+1}^{i} & \equiv\left(E_{t+1}-E_{t}\right) \sum_{q=0}^{\infty} \kappa_{i, 1}^{q} E_{t+1+q}\left[\Delta d_{i, t+2+q}\right] \\
& =\left(E_{t+1}-E_{t}\right) \sum_{q=0}^{\infty} \kappa_{i, 1}^{q}\left(\mu_{i}+\rho_{i}\left(\pi_{t+1+q}-\mu_{\pi}\right)+\xi_{i} \varphi_{t+q} u_{t+1+q}\right) \\
& =\left(\frac{\rho_{i}\left(\phi_{\pi}+\kappa_{i, 1} \xi_{\pi}\right)}{1-\rho_{\pi} \kappa_{i, 1}}+\xi_{i} \varphi_{t}\right) u_{t+1}
\end{aligned}
$$

and depends only on $u_{t+1}$. Iterating equation (OA.11) forward

$$
\begin{aligned}
\pi_{t+j} & =\mu_{\pi}+\rho_{\pi}\left(\pi_{t+j-1}-\mu_{\pi}\right)+\phi_{\pi} u_{t+j}+\xi_{\pi} u_{t+j-1} \\
& =\mu_{\pi}+\rho_{\pi}\left(\rho_{\pi}\left(\pi_{t+j-2}-\mu_{\pi}\right)+\phi_{\pi} u_{t+j-1}+\xi_{\pi} u_{t+j-2}\right)+\phi_{\pi} u_{t+j}+\xi_{\pi} u_{t+j-1} \\
& =\cdots \\
& =\mu_{\pi}+\rho_{\pi}^{j}\left(\pi_{t}-\mu_{\pi}\right)+\phi_{\pi} u_{t+j}-\rho_{\pi}^{j} \phi_{\pi} u_{t}+\sum_{q=0}^{j-1} \rho_{\pi}^{q}\left(\rho_{\pi} \phi_{\pi}+\xi_{\pi}\right) u_{t+j-1-q},
\end{aligned}
$$


gives

$$
\left(E_{t+1}-E_{t}\right) \pi_{t+1+j}=\left\{\begin{array}{cc}
\phi_{\pi} u_{t+1} & , \text { if } j=0 \\
\rho_{\pi}^{j-1}\left(\rho_{\pi} \phi_{\pi}+\xi_{\pi}\right) u_{t+1} & , \text { if } j>0
\end{array} .\right.
$$

It follows that

$$
\begin{aligned}
\left(E_{t+1}-E_{t}\right) \sum_{q=0}^{\infty} \kappa_{i, 1}^{q} \pi_{t+1+j} & =\left(E_{t+1}-E_{t}\right) \pi_{t+1}+\left(E_{t+1}-E_{t}\right) \sum_{q=1}^{\infty} \kappa_{i, 1}^{q} \pi_{t+1+q} \\
& =\left(\phi_{\pi}+\frac{\kappa_{i, 1}\left(\rho_{\pi} \phi_{\pi}+\xi_{\pi}\right)}{1-\kappa_{i, 1} \rho_{\pi}}\right) u_{t+1} .
\end{aligned}
$$

Using equation (OA.20) in equation (OA.9) gives

$$
\begin{aligned}
E_{t+1+j}\left[m_{t+2+j}\right]= & \theta \log \delta+(\theta-1) \kappa_{0}-\gamma E_{t+1+j} \Delta c_{t+2+j}-(\theta-1) w c_{t+1+j} \\
& +(\theta-1) \kappa_{1} E_{t+1+j} w c_{t+2+j} .
\end{aligned}
$$

Plugging (OA.12) and (OA.18) into the last equation and computing expectations

$$
\begin{aligned}
E_{t+1+j}\left[m_{t+2+j}\right]= & \theta \log \delta+(\theta-1) \kappa_{0}-\gamma\left(\mu_{c}+\rho_{c}\left(\pi_{t+1+j}-\mu_{\pi}\right)+\xi_{c} \varphi_{t+j} u_{t+1+j}\right) \\
& -(\theta-1)\left(A_{0}+A_{1}\left(\pi_{t+1+j}-\mu_{\pi}\right)+A_{2} \varphi_{t+j} u_{t+1+j}+A_{3} u_{t+1+j}\right. \\
& \left.+A_{4}\left(\varphi_{t+1+j}-\varphi_{0}\right)+A_{5}\left(\varphi_{t+1+j}^{2}-E\left[\varphi_{t}^{2}\right]\right)\right) \\
& +(\theta-1) \kappa_{1}\left(A_{0}+A_{1}\left(\rho_{\pi}\left(\pi_{t+1+j}-\mu_{\pi}\right)+\xi_{\pi} u_{t+1+j}\right)\right. \\
& \left.+A_{4}\left(E_{t+1+j} \varphi_{t+2+j}-\varphi_{0}\right)+A_{5}\left(E_{t+1+j} \varphi_{t+2+j}^{2}-E\left[\varphi_{t}^{2}\right]\right)\right) \\
= & \theta \log \delta+(\theta-1) \kappa_{0}-\gamma \mu_{c}-(\theta-1) A_{0}+(\theta-1) \kappa_{1} A_{0} \\
& -\frac{\rho_{c}}{\psi}\left(\pi_{t+1+j}-\mu_{\pi}\right) \\
& +\left((\theta-1)\left(\kappa_{1} A_{1} \xi_{\pi}-A_{3}\right)-\frac{1}{\psi} \xi_{c} \varphi_{t+j}\right) u_{t+1+j} \\
& -(\theta-1) A_{4}\left(\varphi_{t+1+j}-\varphi_{0}\right)-(\theta-1) A_{5}\left(\varphi_{t+1+j}^{2}-E\left[\varphi_{t}^{2}\right]\right) \\
& +(\theta-1) \kappa_{1} A_{4}\left(E_{t+1+j} \varphi_{t+2+j}-\varphi_{0}\right) \\
& +(\theta-1) \kappa_{1} A_{5}\left(E_{t+1+j} \varphi_{t+2+j}^{2}-E\left[\varphi_{t}^{2}\right]\right) .
\end{aligned}
$$


$N_{m, t+1}^{i}$ can now be computed using equations (OA.77) and (OA.78)

$$
\begin{aligned}
N_{m, t+1}^{i}= & \left(E_{t+1}-E_{t}\right) \sum_{q=0}^{\infty} \kappa_{i, 1}^{q} E_{t+1+j}\left[m_{t+2+j}\right] \\
= & -\frac{\rho_{c}}{\psi}\left(E_{t+1}-E_{t}\right) \sum_{q=0}^{\infty} \kappa_{i, 1}^{q} \pi_{t+1+j} \\
& +\left(E_{t+1}-E_{t}\right) \sum_{q=0}^{\infty} \kappa_{i, 1}^{q}\left((\theta-1)\left(\kappa_{1} A_{1} \xi_{\pi}-A_{3}\right)-\frac{1}{\psi} \xi_{c} \varphi_{t+j}\right) u_{t+1+j} \\
& +\left(E_{t+1}-E_{t}\right) \sum_{q=0}^{\infty} \kappa_{i, 1}^{q}\left[-(\theta-1) A_{4}\left(\varphi_{t+1+j}-\varphi_{0}\right)-(\theta-1) A_{5}\left(\varphi_{t+1+j}^{2}-E\left[\varphi_{t}^{2}\right]\right)\right] \\
& +\left(E_{t+1}-E_{t}\right) \sum_{q=0}^{\infty} \kappa_{i, 1}^{q}\left[(\theta-1) \kappa_{1} A_{4}\left(E_{t+1+j} \varphi_{t+2+j}-\varphi_{0}\right)\right. \\
& \left.+(\theta-1) \kappa_{1} A_{5}\left(E_{t+1+j} \varphi_{t+2+j}^{2}-E\left[\varphi_{t}^{2}\right]\right)\right] \\
= & -\frac{\rho_{c}}{\psi} \frac{\kappa_{i, 1}\left(\rho_{\pi} \phi_{\pi}+\xi_{\pi}\right)}{1-\kappa_{i, 1} \rho_{\pi}} u_{t+1} \\
& +\left((\theta-1)\left(\kappa_{1} A_{1} \xi_{\pi}-A_{3}\right)-\frac{\rho_{c}}{\psi} \phi_{\pi}-\frac{1}{\psi} \xi_{c} \varphi_{t}\right) u_{t+1} \\
& +\left(E_{t+1}-E_{t}\right) \sum_{q=0}^{\infty} \kappa_{i, 1}^{q}\left[-(\theta-1) A_{4}\left(\varphi_{t+1+j}-\varphi_{0}\right)-(\theta-1) A_{5}\left(\varphi_{t+1+j}^{2}-E\left[\varphi_{t}^{2}\right]\right)\right] \\
& +\left(E_{t+1}-E_{t}\right) \sum_{q=0}^{\infty} \kappa_{i, 1}^{q}\left[(\theta-1) \kappa_{1} A_{4}\left(E_{t+1+j} \varphi_{t+2+j}-\varphi_{0}\right)\right. \\
& \left.+(\theta-1) \kappa_{1} A_{5}\left(E_{t+1+j} \varphi_{t+2+j}^{2}-E\left[\varphi_{t}^{2}\right]\right)\right] .
\end{aligned}
$$

By equation (OA.13), the innovations $\left(E_{t+1}-E_{t}\right) \varphi_{t+j+2}$ and $\left(E_{t+1}-E_{t}\right) \varphi_{t+j+2}^{2}$ only depend on the shock $w_{t+1}$, so we can write equation $(\mathrm{OA} .79)$ as

$$
\begin{aligned}
N_{m, t+1}^{i}= & -\frac{\rho_{c}}{\psi} \frac{\kappa_{i, 1}\left(\rho_{\pi} \phi_{\pi}+\xi_{\pi}\right)}{1-\kappa_{i, 1} \rho_{\pi}} u_{t+1} \\
& +\left((\theta-1)\left(\kappa_{1} A_{1} \xi_{\pi}-A_{3}\right)-\frac{\rho_{c}}{\psi} \phi_{\pi}-\frac{1}{\psi} \xi_{c} \varphi_{t}\right) u_{t+1} \\
& +R\left(w_{t+1}\right)
\end{aligned}
$$

where $R(\cdot)$ is a function of $w_{t+1}$ but not of $\left(\eta_{t+1}, u_{t+1}\right)$. By direct computation, equation (OA.14) implies that

$$
\begin{aligned}
N_{D, t+1}^{i} & \equiv\left(E_{t+1}-E_{t}\right) \Delta d_{i, t+1} \\
& =\sigma_{i} \eta_{t+1}
\end{aligned}
$$

and thus $N_{D, t+1}^{i}$ depends only on the shock $\eta_{t+1}$. Finally, by noting that $N_{R I S K, t+1}^{i}$ depends only on variances and covariances and that $\varphi_{t}$ is the only source of heteroskedasticity in the model, $N_{R I S K, t+1}^{i}$ depends only 
on the shock $w_{t+1}$.

\subsection{Regression from Table 7}

The goal of this section is to compute the coefficient $L_{\beta_{\Pi} \times N R C, m}$ in regression (9) when the regression is run in the model (instead of in the data, as is done in Table 7).

\subsection{Preliminary Calculations}

Iterating equation (OA.13) forward, we have that for $j>1$

$$
\begin{aligned}
\varphi_{t+j}-\varphi_{0} & =v\left(\varphi_{t+j-1}-\varphi_{0}\right)+\sigma_{w} w_{t+j} \\
& =v^{2}\left(\varphi_{t+j-2}-\varphi_{0}\right)+v \sigma_{w} w_{t+j-1}+\sigma_{w} w_{t+j} \\
& =\ldots \\
& =v^{j}\left(\varphi_{t}-\varphi_{0}\right)+v^{j-1} \sigma_{w} w_{t+1}+\ldots+v \sigma_{w} w_{t+j-1}+\sigma_{w} w_{t+j} \\
& =v^{j}\left(\varphi_{t}-\varphi_{0}\right)+\sum_{q=0}^{j-1} v^{q} \sigma_{w} w_{t+j-q} .
\end{aligned}
$$

Squaring both sides gives

$$
\begin{aligned}
\varphi_{t+j}^{2}= & \left(\varphi_{0}+v^{j}\left(\varphi_{t}-\varphi_{0}\right)\right)^{2}+2\left(\varphi_{0}+v^{j}\left(\varphi_{t}-\varphi_{0}\right)\right) \sum_{q=0}^{j-1} v^{q} \sigma_{w} w_{t+j-q} \\
& +\left(\sum_{q=0}^{j-1} v^{q} \sigma_{w} w_{t+j-q}\right)^{2} .
\end{aligned}
$$

Taking expectations conditional on time $t$ in equations (OA.76), ( OA.82) and (OA.83) gives

$$
\begin{aligned}
& E_{t}\left[\pi_{t+s-1}\right]-\mu_{\pi}=\left\{\begin{array}{cl}
\pi_{t}-\mu_{\pi} & , \text { if } s=1 \\
\rho_{\pi}^{s-1}\left(\pi_{t}-\mu_{\pi}\right)+\rho_{\pi}^{s-2} \xi_{\pi} u_{t} & , \text { if } s>1
\end{array}\right. \text {, } \\
& E_{t}\left[\varphi_{t+s-1}-\varphi_{0}\right]=\left\{\begin{array}{cc}
\varphi_{t}-\varphi_{0} & , \text { if } s=1 \\
v^{s-1}\left(\varphi_{t}-\varphi_{0}\right) & , \text { if } s>1
\end{array},\right. \\
& E_{t}\left[\varphi_{t+s-1}^{2}-E\left[\varphi_{t}^{2}\right]\right]=\left\{\begin{array}{cl}
\varphi_{t}^{2}-E\left[\varphi_{t}^{2}\right] & \text { if } s=1 \\
\left(\varphi_{0}+v^{s-1}\left(\varphi_{t}-\varphi_{0}\right)\right)^{2}+\sigma_{w}^{2} \frac{v^{2(s-1)}-1}{\nu^{2}-1}-E\left[\varphi_{t}^{2}\right], & \text { if } s>1
\end{array} .\right.
\end{aligned}
$$

Because $\varphi_{\tau}$ and $u_{t}$ are independent for all $t, \tau$, we have

$$
E_{t}\left[\varphi_{t+s-2} u_{t+s-1}\right]=\left\{\begin{array}{cl}
\varphi_{t-1} u_{t} & , \text { if } s=1 \\
0 & , \text { if } s>1
\end{array} .\right.
$$


Since $u_{t}$ and $w_{t}$ are independent, we have

$$
\begin{aligned}
\operatorname{Cov}\left(\pi_{t}, \varphi_{t}\right) & =0, \\
\operatorname{Cov}\left(u_{t}, \varphi_{t}\right) & =0, \\
\operatorname{Cov}\left(\varphi_{t-1} u_{t}, \varphi_{t}\right) & =E\left[\varphi_{t-1} u_{t}\left(\varphi_{t}-\varphi_{0}\right)\right] \\
& =E\left[E_{t}\left[\varphi_{t-1} u_{t}\left(\varphi_{t}-\varphi_{0}\right)\right]\right] \\
& =E\left[\varphi_{t-1} E_{t}\left[u_{t}\left(\varphi_{t}-\varphi_{0}\right)\right]\right] \\
& =E\left[\varphi_{t-1} E_{t}\left[u_{t}\right] E_{t}\left[\varphi_{t}-\varphi_{0}\right]\right] \\
& =0,
\end{aligned}
$$

and

$$
\begin{aligned}
\operatorname{Cov}\left(\pi_{t}, \varphi_{t}^{2}\right)= & 0 \\
\operatorname{Cov}\left(u_{t}, \varphi_{t}^{2}\right)= & 0, \\
\operatorname{Cov}\left(\varphi_{t-1} u_{t},\left(\varphi_{t}-\varphi_{0}\right)^{2}\right)= & \operatorname{Cov}\left(\varphi_{t-1} u_{t}, \varphi_{0}^{2}-2 \varphi_{0} \varphi_{t}+\varphi_{t}^{2}\right) \\
= & \operatorname{Cov}\left(\varphi_{t-1} u_{t}, \varphi_{0}^{2}\right) \\
& -2 \varphi_{0} \operatorname{Cov}\left(\varphi_{t-1} u_{t}, \varphi_{t}\right) \\
& +\operatorname{Cov}\left(\varphi_{t-1} u_{t}, \varphi_{t}^{2}\right) \\
= & \operatorname{Cov}\left(\varphi_{t-1} u_{t}, \varphi_{t}^{2}\right) \\
= & E\left[\varphi_{t-1} u_{t}\left(\varphi_{t}^{2}-E\left[\varphi_{0}^{2}\right]\right)\right] \\
= & E\left[\varphi_{t-1} E_{t}\left[u_{t}\left(\varphi_{t}^{2}-E\left[\varphi_{0}^{2}\right]\right)\right]\right] \\
= & E\left[\varphi_{t-1} E_{t}\left[u_{t}\right] E_{t}\left[\varphi_{t}^{2}-E\left[\varphi_{0}^{2}\right]\right]\right] \\
= & 0 .
\end{aligned}
$$

Define $\tilde{\theta}_{t}^{(12)}$ to be the nominal-real covariance $\theta_{t}^{(12)}$ normalized to have mean equal to zero and standard deviation equal to one, and $\tilde{\beta}_{u, i t}$ to be the inflation beta $\beta_{u, i t}$ minus its unconditional mean:

$$
\begin{aligned}
& \tilde{\theta}_{t}^{(12)} \equiv \frac{\theta_{t}^{(12)}-E\left[\theta_{t}^{(12)}\right]}{\operatorname{std}\left(\theta_{t}^{(12)}\right)}, \\
& \tilde{\beta}_{u, i t} \equiv \beta_{u, i t}-E\left[\beta_{u, i t}\right] .
\end{aligned}
$$


Using equations (19), (OA.13) and (OA.53), we find

$$
\begin{aligned}
& \tilde{\theta}_{t}^{(12)}=\frac{\operatorname{sign}\left(\xi_{c}\right)}{\operatorname{sign}\left(\phi_{\pi}\right)} \frac{\sqrt{1-\nu^{2}}}{\left|\sigma_{w}\right|}\left(\varphi_{t}-\varphi_{0}\right), \\
& \tilde{\beta}_{u, i t}=\kappa_{i, 1}\left(\xi_{i}-\frac{\xi_{c}}{\psi}\right)\left(\varphi_{t}-\varphi_{0}\right) .
\end{aligned}
$$

and therefore

$$
\tilde{\beta}_{u, i t} \times \tilde{\theta}_{t}^{(12)}=\frac{\operatorname{sign}\left(\xi_{c}\right)}{\operatorname{sign}\left(\phi_{\pi}\right)} \frac{\kappa_{i, 1} \sqrt{1-\nu^{2}}}{\left|\sigma_{w}\right|}\left(\xi_{i}-\frac{\xi_{c}}{\psi}\right)\left(\varphi_{t}-\varphi_{0}\right)^{2} .
$$

\subsection{Running the Regression}

We let the asset $i=m$ be the asset that represents the aggregate stock market in the model. We can write $(\log )$ returns cumulated from $t+1$ to $t+12$ as the sum of expected and unexpected components as of time $t$

$$
\begin{aligned}
r_{m, t+1: t+12} & =\sum_{s=1}^{12} r_{m, t+s} \\
& =\sum_{s=1}^{12} E_{t}\left[r_{m, t+s}\right]+\sum_{s=1}^{12}\left(r_{m, t+s}-E_{t}\left[r_{m, t+s}\right]\right) .
\end{aligned}
$$

Using equations (OA.28)-(OA.31), (OA.51) and (OA.52) gives

$$
\begin{aligned}
\sum_{s=1}^{12} E_{t}\left[r_{m, t+1: t+12}\right]= & R_{0}+R_{1}\left(\pi_{t}-\mu_{\pi}\right)+R_{2} \varphi_{t-1} u_{t}+R_{3} u_{t} \\
& +R_{4}\left(\varphi_{t}-\varphi_{0}\right)+R_{5}\left(\varphi_{t}^{2}-E\left[\varphi_{t}^{2}\right]\right), \\
\sum_{s=1}^{12}\left(r_{m, t+s}-E_{t}\left[r_{m, t+s}\right]\right)= & \sum_{s=1}^{12}\left(\beta_{u, m, t+s-1} u_{t+s}+\beta_{w, m} w_{t+s}\right) \\
& +\sum_{s=1}^{12}\left(\beta_{2 w, m}\left(\frac{w_{t+s}^{2}-1}{\sqrt{2}}\right)+\beta_{\eta, m} \eta_{t+s}\right)
\end{aligned}
$$

where the constants $R_{i}$ are given by

$$
\begin{aligned}
& R_{0}=12\left(\kappa_{m, 0}+\mu_{m}-D_{m, 0}\left(1-\kappa_{m, 1}\right)\right) \\
& R_{1}=\left(\rho_{m}-D_{m, 1}\left(1-\kappa_{m, 1} \rho_{\pi}\right)\right)\left(\sum_{s=1}^{12} \rho_{\pi}^{s-1}\right), \\
& R_{2}=\xi_{m}-D_{m, 2}, \\
& R_{3}=\xi_{\pi}\left(\kappa_{m, 1} \sum_{s=1}^{12} \rho_{\pi}^{s-1}+\frac{1-\rho_{m}}{\rho_{\pi}}\left(1-\sum_{s=1}^{12} \rho_{\pi}^{s-1}\right)\right)-D_{m, 3}, \\
& R_{4}=-2 \varphi_{0} v D_{m, 5} v^{s-2} \sum_{s=1}^{12}\left(1-v^{s-1}-\kappa_{m, 1} v\left(1-v^{s}\right)\right)-D_{m, 4}\left(1-v \kappa_{m, 1}\right)\left(\sum_{s=1}^{12} v^{s-1}\right), \\
& R_{5}=-D_{m, 5}\left(1-v^{2} \kappa_{m, 1}\right)\left(\sum_{s=1}^{12} v^{2(s-1)}\right) .
\end{aligned}
$$


Defining

$$
\begin{aligned}
\varepsilon_{t+1: t+12} & \equiv \sum_{s=1}^{12}\left(\beta_{u, m, t+s-1} u_{t+s}+\beta_{w, m} w_{t+s}+\beta_{2 w, m}\left(\frac{w_{t+s}^{2}-1}{\sqrt{2}}\right)+\beta_{\eta, m} \eta_{t+s}\right), \\
\varkappa_{t} & \equiv R_{1}\left(\pi_{t}-\mu_{\pi}\right)+R_{2} \varphi_{t-1} u_{t}+R_{3} u_{t}, \\
L_{2} & \equiv-\frac{1}{\kappa_{m, 1}} \frac{\operatorname{sign}\left(\phi_{\pi}\right)}{\operatorname{sign}\left(\xi_{c}\right)} \frac{\left|\sigma_{w}\right|}{\sqrt{1-\nu^{2}}} \frac{R_{5}}{\xi_{c} / \psi-\xi_{m}}, \\
L_{1} & \equiv R_{4}+2 R_{5} \varphi_{0}, \\
L_{0} & \equiv R_{0}-\frac{\sigma_{w}^{2}}{1-v^{2}} R_{5},
\end{aligned}
$$

and using equations (OA.88), (OA.90) and (OA.91), we can write equation (OA.89) as

$$
r_{m, t+1: t+12}=L_{0}+L_{1}\left(\varphi_{t}-\varphi_{0}\right)+L_{2}\left(\tilde{\beta}_{u, m t} \times \tilde{\theta}_{t}^{(12)}\right)+\varkappa_{t}+\varepsilon_{t+1: t+12}
$$

Under the null of the model, running regression (OA.94) with or without including the regressors $\varkappa_{t}$ gives the same estimate $\hat{L}_{0}, \hat{L}_{1}, \hat{L}_{2}$ for $L_{0}, L_{1}, L_{2}$ since $\varkappa_{t}$ is uncorrelated to the other regressors by equations (OA.84)-(OA.85), and uncorrelated to the error term by the law of iterated expectations and the fact that $E_{t}\left[\varepsilon_{t+1: t+12}\right]=0$ :

$$
\begin{aligned}
E\left[\varkappa_{t} \varepsilon_{t+1: t+12}\right] & =E\left[E_{t}\left[\varkappa_{t} \varepsilon_{t+1: t+12}\right]\right] \\
& =E\left[\varkappa_{t} E_{t}\left[\varepsilon_{t+1: t+12}\right]\right] \\
& =0 .
\end{aligned}
$$

Since we are interested in $\hat{L}_{2}$, we therefore run regression (OA.94) in the model without including $\varkappa_{t}$

$$
r_{m, t+1: t+12}=L_{0}+L_{1}\left(\varphi_{t}-\varphi_{0}\right)+L_{2}\left(\tilde{\beta}_{u, m t} \times \tilde{\theta}_{t}^{(12)}\right)+\varepsilon_{t+1: t+12}
$$

and obtain estimates $\hat{L}_{0}, \hat{L}_{1}, \hat{L}_{2}$. In the data, we run the regression in equation (9) reproduced next for convenience:

$$
r_{m, t+1: t+12}=L_{0, m}+L_{\beta_{\Pi}, m} \tilde{\beta}_{u, m t}+L_{N R C, m} \tilde{\theta}_{t}^{(12)}+L_{\beta_{\Pi} \times N R C, m}\left(\tilde{\beta}_{u, m t} \times \tilde{\theta}_{t}^{(12)}\right)+\varepsilon_{t+1: t+12} .
$$

We now show that, under the null of the model, the estimate $\hat{L}_{\beta_{\Pi} \times N R C, m}$ from regression (OA.96) is the same as the estimate $\hat{L}_{2}$ obtained from regression (OA.95). By equations (OA.86)-(OA.87),

$$
\begin{aligned}
L_{0, m}+L_{\beta_{\Pi}, m} \tilde{\beta}_{u, m t}+L_{N R C, m} \tilde{\theta}_{t}^{(12)}= & L_{0, m}+L_{\beta_{\Pi}, m} \kappa_{i, 1}\left(\xi_{i}-\frac{\xi_{c}}{\psi}\right)\left(\varphi_{t}-\varphi_{0}\right) \\
& +L_{N R C, m} \frac{\operatorname{sign}\left(\xi_{c}\right)}{\operatorname{sign}\left(\phi_{\pi}\right)} \frac{\sqrt{1-\nu^{2}}}{\left|\sigma_{w}\right|}\left(\varphi_{t}-\varphi_{0}\right) \\
= & L_{0, m} \\
& +\left[L_{\beta_{\Pi}, m} \kappa_{i, 1}\left(\xi_{i}-\frac{\xi_{c}}{\psi}\right)+L_{N R C, m} \frac{\operatorname{sign}\left(\xi_{c}\right)}{\operatorname{sign}\left(\phi_{\pi}\right)} \frac{\sqrt{1-\nu^{2}}}{\left|\sigma_{w}\right|}\right]\left(\varphi_{t}-\varphi_{0}\right),
\end{aligned}
$$


so regression (OA.96) can be written as

$$
\begin{aligned}
r_{m, t+1: t+12}= & L_{0, m}+\left[L_{\beta_{\Pi}, m} \kappa_{i, 1}\left(\xi_{i}-\frac{\xi_{c}}{\psi}\right)+L_{N R C, m} \frac{\operatorname{sign}\left(\xi_{c}\right)}{\operatorname{sign}\left(\phi_{\pi}\right)} \frac{\sqrt{1-\nu^{2}}}{\left|\sigma_{w}\right|}\right]\left(\varphi_{t}-\varphi_{0}\right) \\
& +L_{\beta_{\Pi} \times N R C, m}\left(\tilde{\beta}_{u, m t} \times \tilde{\theta}_{t}^{(12)}\right)+\varepsilon_{t+1: t+12},
\end{aligned}
$$

where we can then identify

$$
\begin{aligned}
& \hat{L}_{0}=\hat{L}_{0, m}, \\
& \hat{L}_{1}=\hat{L}_{\beta_{\Pi}, m} \kappa_{i, 1}\left(\xi_{i}-\frac{\xi_{c}}{\psi}\right)+\hat{L}_{N R C, m} \frac{\operatorname{sign}\left(\xi_{c}\right)}{\operatorname{sign}\left(\phi_{\pi}\right)} \frac{\sqrt{1-\nu^{2}}}{\left|\sigma_{w}\right|}, \\
& \hat{L}_{2}=\hat{L}_{\beta_{\Pi} \times N R C, m} .
\end{aligned}
$$

Note that although $\hat{L}_{\beta_{\Pi}, m}$ and $\hat{L}_{N R C, m}$ cannot be separately identified in the model by running regression (OA.95), the coefficient we seek, $\hat{L}_{\beta_{\Pi} \times N R C, m}$, is identified by $\hat{L}_{2}$. Plugging (OA.93) into (OA.92), we find

$$
\begin{aligned}
L_{2} & =L_{\beta_{\Pi} \times N R C, m} \\
& =\frac{1}{\kappa_{m, 1}} \frac{\operatorname{sign}\left(\phi_{\pi}\right)}{\operatorname{sign}\left(\xi_{c}\right)} \frac{\left|\sigma_{w}\right|}{\sqrt{1-\nu^{2}}} \frac{D_{m, 5}\left(1-v^{2} \kappa_{m, 1}\right)\left(\sum_{s=1}^{12} v^{2(s-1)}\right)}{\xi_{c} / \psi-\xi_{m}} .
\end{aligned}
$$

Note also that

$$
\begin{aligned}
\frac{\operatorname{cov}\left(r_{m, t+1: t+12}, \tilde{\beta}_{u, i t} \times \tilde{\theta}_{t}^{(12)}\right)}{\operatorname{var}\left(\tilde{\beta}_{u, i t} \times \tilde{\theta}_{t}^{(12)}\right)}= & \frac{\operatorname{cov}\left(L_{1}\left(\varphi_{t}-\varphi_{0}\right), \tilde{\beta}_{u, m t} \times \tilde{\theta}_{t}^{(12)}\right)}{\operatorname{var}\left(\tilde{\beta}_{u, i t} \times \tilde{\theta}_{t}^{(12)}\right)} \\
& +\frac{\operatorname{cov}\left(L_{2}, \tilde{\beta}_{u, m t} \times \tilde{\theta}_{t}^{(12)}\right)}{\operatorname{var}\left(\tilde{\beta}_{u, i t} \times \tilde{\theta}_{t}^{(12)}\right)} \\
& +\frac{\operatorname{cov}\left(L_{0}+\varkappa_{t}+\varepsilon_{t+1: t+12}, \tilde{\beta}_{u, i t} \times \tilde{\theta}_{t}^{(12)}\right)}{\operatorname{var}\left(\tilde{\beta}_{u, i t} \times \tilde{\theta}_{t}^{(12)}\right)} \\
= & L_{1} \frac{\operatorname{cov}\left(\varphi_{t}-\varphi_{0}, \tilde{\beta}_{u, i t} \times \tilde{\theta}_{t}^{(12)}\right)}{\operatorname{var}\left(\tilde{\beta}_{u, i t} \times \tilde{\theta}_{t}^{(12)}\right)}+L_{2}+\frac{\operatorname{cov}\left(\varepsilon_{t+1: t+12}, \tilde{\beta}_{u, i t} \times \tilde{\theta}_{t}^{(12)}\right)}{\operatorname{var}\left(\tilde{\beta}_{u, i t} \times \tilde{\theta}_{t}^{(12)}\right)} \\
= & \frac{\operatorname{sign}\left(\xi_{c}\right)}{\operatorname{sign}\left(\phi_{\pi}\right)} \frac{\kappa_{i, 1} \sqrt{1-\nu^{2}}}{\left|\sigma_{w}\right|}\left(\xi_{i}-\frac{\xi_{c}}{\psi}\right) L_{1} \frac{\operatorname{cov}\left(\varphi_{t}-\varphi_{0},\left(\varphi_{t}-\varphi_{0}\right)^{2}\right)}{\operatorname{var}\left(\tilde{\beta}_{u, i t} \times \tilde{\theta}_{t}^{(12)}\right)} \\
& +L_{2}+\frac{\operatorname{sign}\left(\xi_{c}\right)}{\operatorname{sign}\left(\phi_{\pi}\right)} \frac{\kappa_{i, 1} \sqrt{1-\nu^{2}}}{\left|\sigma_{w}\right|}\left(\xi_{i}-\frac{\xi_{c}}{\psi}\right) \frac{\operatorname{cov}\left(\varepsilon_{t+1: t+12},\left(\varphi_{t}-\varphi_{0}\right)^{2}\right)}{\operatorname{vvar}\left(\tilde{\beta}_{u, i t} \times \tilde{\theta}_{t}^{(12)}\right)} \\
= & L_{2}, \quad
\end{aligned}
$$


since

$$
\begin{gathered}
\operatorname{cov}\left(\varphi_{t}-\varphi_{0},\left(\varphi_{t}-\varphi_{0}\right)^{2}\right)=0, \\
\frac{\operatorname{cov}\left(\varepsilon_{t+1: t+12},\left(\varphi_{t}-\varphi_{0}\right)^{2}\right)}{\operatorname{var}\left(\left(\varphi_{t}-\varphi_{0}\right)^{2}\right)}=0 .
\end{gathered}
$$

It then follows that $L_{\beta_{\Pi} \times N R C, m}$ can actually be computed from a univariate regression of $r_{m, t+1: t+12}$ on $\tilde{\beta}_{u, i t} \times \tilde{\theta}_{t}^{(12)}$ since $\tilde{\beta}_{u, i t} \times \tilde{\theta}_{t}^{(12)}$ is uncorrelated to the rest of the regressors.

\subsection{Unconditional and Conditional Predictability of Consumption with Inflation}

We show how to compute, in the model, the coefficients $d_{0}^{u, K}, d_{1}^{u, K}, d_{0}^{c, K}, d_{1}^{c, K}$ and the $R^{2}$ of the regressions in equations (1) and (3) and Table 2. Using equation (OA.76), we have

$$
\begin{aligned}
\Delta c_{t+1+j}= & \mu_{c}+\rho_{c}\left(\pi_{t+j}-\mu_{\pi}\right)+\sigma_{c} \eta_{t+1+j}+\xi_{c} \varphi_{t-1+j} u_{t+j} \\
= & \mu_{c}+\rho_{c}\left(\pi_{t+j}-\mu_{\pi}\right)+\sigma_{c} \eta_{t+1+j}+\xi_{c} \varphi_{t-1+j} u_{t+j} \\
= & \mu_{c}+\rho_{c}\left(\rho_{\pi}^{j}\left(\pi_{t}-\mu_{\pi}\right)+\phi_{\pi} u_{t+j}-\rho_{\pi}^{j} \phi_{\pi} u_{t}+\sum_{q=0}^{j-1} \rho_{\pi}^{q}\left(\rho_{\pi} \phi_{\pi}+\xi_{\pi}\right) u_{t+j-1-q}\right) \\
& +\sigma_{c} \eta_{t+1+j}+\xi_{c}\left(\varphi_{0}+v^{j}\left(\varphi_{t-1}-\varphi_{0}\right)+\sum_{q=0}^{j-1} v^{q} \sigma_{w} w_{t-1+j-q}\right) u_{t+j},
\end{aligned}
$$

and therefore

$$
\begin{aligned}
E_{t}\left[\Delta c_{t+1+j}\right]= & \mu_{c}+\rho_{c}\left(\rho_{\pi}^{j}\left(\pi_{t}-\mu_{\pi}\right)+\phi_{\pi} E_{t}\left[u_{t+j}\right]-\rho_{\pi}^{j} \phi_{\pi} E_{t}\left[u_{t}\right]+\sum_{q=0}^{j-1} \rho_{\pi}^{q}\left(\rho_{\pi} \phi_{\pi}+\xi_{\pi}\right) E_{t}\left[u_{t+j-1-q}\right]\right) \\
& +\sigma_{c} E_{t}\left[\eta_{t+1+j}\right]+\xi_{c}\left(\varphi_{0}+v^{j}\left(\varphi_{t-1}-\varphi_{0}\right)+\sum_{q=0}^{j-1} v^{q} \sigma_{w} E_{t}\left[w_{t-1+j-q}\right]\right) E_{t}\left[u_{t+j}\right] \\
= & \mu_{c}+\rho_{c}\left(\rho_{\pi}^{j}\left(\pi_{t}-\mu_{\pi}\right)-\rho_{\pi}^{j} \phi_{\pi} u_{t}+\left(\rho_{\pi} \phi_{\pi}+\xi_{\pi}\right) E_{t}\left[u_{t+j-1}\right]\right) \\
& +\rho_{c} \sum_{q=1}^{j-1} \rho_{\pi}^{q}\left(\rho_{\pi} \phi_{\pi}+\xi_{\pi}\right) E_{t}\left[u_{t+j-1-q}\right] \\
= & \mu_{c}+\rho_{c}\left(\rho_{\pi}^{j}\left(\pi_{t}-\mu_{\pi}\right)-\rho_{\pi}^{j} \phi_{\pi} u_{t}+\rho_{\pi}^{j-1}\left(\rho_{\pi} \phi_{\pi}+\xi_{\pi}\right) u_{t}\right) .
\end{aligned}
$$


Using equations (OA.11)-(OA.13), (OA.98), (OA.100), and the law of total covariance

$$
\begin{aligned}
& \operatorname{Cov}\left(\Delta c_{t+1+j}, \pi_{t+1}\right)=E\left[\operatorname{Cov}_{t}\left(\Delta c_{t+1+j}, \pi_{t+1}\right)\right]+\operatorname{cov}\left(E_{t}\left[\Delta c_{t+1+j}\right], E_{t}\left[\pi_{t+1}\right]\right) \\
& =\left\{\begin{array}{cc}
\rho_{\pi} \rho_{c} \operatorname{Var}\left(\pi_{t}\right)+\varphi_{0} \xi_{c}\left(\xi_{\pi}+\phi_{\pi} \rho_{\pi}\right)+\rho_{c} \xi_{\pi} \phi_{\pi} & , \text { if } j=0 \\
\phi_{\pi}^{2} \rho_{c}+\phi_{\pi} \xi_{c} \varphi_{0} & , \text { if } j=1 \\
\phi_{\pi} \rho_{c} \rho_{\pi}^{j-2}\left(\xi_{\pi}+\phi_{\pi} \rho_{\pi}\right) & , \text { if } j>1
\end{array}\right. \\
& +\left\{\begin{array}{cc}
\rho_{\pi} \rho_{c} \operatorname{Var}\left(\pi_{t}\right)+\rho_{c} \xi_{\pi} \phi_{\pi}+\varphi_{0} \xi_{c}\left(\xi_{\pi}+\phi_{\pi} \rho_{\pi}\right) & , \text { if } j=0 \\
\rho_{\pi}^{j+1} \rho_{c} \operatorname{Var}\left(\pi_{t}\right)+\rho_{c} \xi_{\pi} \rho_{\pi}^{j-1}\left(\xi_{\pi}+2 \phi_{\pi} \rho_{\pi}\right) & , \text { if } j>0
\end{array}\right. \\
& =\left\{\begin{array}{cc}
2\left(\rho_{\pi} \rho_{c} \operatorname{Var}\left(\pi_{t}\right)+\varphi_{0} \xi_{c}\left(\xi_{\pi}+\phi_{\pi} \rho_{\pi}\right)+\rho_{c} \phi_{\pi} \xi_{\pi}\right) & , \text { if } j=0 \\
\rho_{c} \operatorname{Var}\left(\pi_{t}\right)+\xi_{c} \varphi_{0} \phi_{\pi} & , \text { if } j=1 \\
\rho_{c} \rho_{\pi}^{j-1} \operatorname{Var}\left(\pi_{t}\right)+\rho_{\pi}^{j-2} \rho_{c} \phi_{\pi} \xi_{\pi} & , \text { if } j>1
\end{array} .\right.
\end{aligned}
$$

It follows that

$$
\begin{aligned}
d_{1}^{u, K}= & \frac{\operatorname{Cov}\left(\sum_{j=0}^{K-1} \Delta c_{t+1+j}, \pi_{t}\right)}{\operatorname{Var}\left(\pi_{t}\right)} \\
= & \frac{\sum_{j=0}^{K-1} \operatorname{Cov}\left(\Delta c_{t+1+j}, \pi_{t}\right)}{\operatorname{Var}\left(\pi_{t}\right)} \\
= & \frac{\operatorname{Cov}\left(\Delta c_{t+1}, \pi_{t}\right)}{\operatorname{Var}\left(\pi_{t}\right)}+\frac{\operatorname{Cov}\left(\Delta c_{t+2}, \pi_{t}\right)}{\operatorname{Var}\left(\pi_{t}\right)}+\frac{\sum_{j=2}^{K-1} \operatorname{Cov}\left(\Delta c_{t+1+j}, \pi_{t}\right)}{\operatorname{Var}\left(\pi_{t}\right)} \\
= & \frac{2\left(\rho_{\pi} \rho_{c} \operatorname{Var}\left(\pi_{t}\right)+\varphi_{0} \xi_{c}\left(\xi_{\pi}+\phi_{\pi} \rho_{\pi}\right)+\rho_{c} \phi_{\pi} \xi_{\pi}\right)}{\operatorname{Var}\left(\pi_{t}\right)}+\frac{\rho_{c} \operatorname{Var}\left(\pi_{t}\right)+\xi_{c} \varphi_{0} \phi_{\pi}}{\operatorname{Var}\left(\pi_{t}\right)} \\
& +\frac{\sum_{j=2}^{K-1}\left(\rho_{c} \rho_{\pi}^{j-1} \operatorname{Var}\left(\pi_{t}\right)+\rho_{\pi}^{j-2} \rho_{c} \phi_{\pi} \xi_{\pi}\right)}{\operatorname{Var}\left(\pi_{t}\right)} \\
= & 2 \rho_{\pi} \rho_{c}+\rho_{c}+\frac{\varphi_{0} \xi_{c}\left(\phi_{\pi}+2 \phi_{\pi} \rho_{\pi}+2 \xi_{\pi}\right)+\rho_{c} \phi_{\pi} \xi_{\pi}}{\operatorname{Var}\left(\pi_{t}\right)} \\
& +\rho_{c} \sum_{j=2}^{K-1} \rho_{\pi}^{j-1}+\frac{\rho_{c} \phi_{\pi} \xi_{\pi}}{\operatorname{Var}\left(\pi_{t}\right)} \sum_{j=2}^{K-1} \rho_{\pi}^{j-2} \\
= & \rho_{c}\left(\frac{1-\rho_{\pi}^{K}}{1-\rho_{\pi}}\right)\left(1+\frac{\xi_{\pi} \phi_{\pi}}{\operatorname{Var}\left(\pi_{t}\right)}\left(\frac{1-\rho_{\pi}^{K-1}}{1-\rho_{\pi}^{K}}\right)\right)+\frac{\xi_{c} \phi_{\pi}}{\operatorname{Var}\left(\pi_{t}\right)} \varphi_{0},
\end{aligned}
$$

and

$$
\begin{aligned}
d_{0}^{u, K} & =E\left[\sum_{j=0}^{K-1} \Delta c_{t+1+j}-d_{1}^{u, K} \pi_{t}\right] \\
& =\sum_{j=0}^{K-1} E\left[\Delta c_{t+1+j}\right]-d_{1}^{u, K} E\left[\pi_{t}\right] \\
& =K \mu_{c}-d_{1}^{u, K} \mu_{\pi} .
\end{aligned}
$$


Now we turn to the conditional regression of Table 2. Because of the way we constructed $b_{t}^{K}$, we can write

$$
\sum_{j=0}^{K-1} \Delta c_{t+1+j}=a_{t}^{K}+b_{t}^{K} \pi_{t}+\varepsilon_{t+K},
$$

where, using equations (OA.11) and (OA.100), we have

$$
\begin{aligned}
a_{t}^{K}= & E_{t}\left[\sum_{j=1}^{K} \Delta c_{t+1+j}-b_{t}^{K} \pi_{t+1}\right] \\
= & \sum_{j=1}^{K} E_{t}\left[\Delta c_{t+1+j}\right]-b_{t}^{K} E_{t}\left[\pi_{t+1}\right] \\
= & \sum_{j=1}^{K}\left(\mu_{c}+\rho_{c}\left(\rho_{\pi}^{j}\left(\pi_{t}-\mu_{\pi}\right)-\rho_{\pi}^{j} \phi_{\pi} u_{t}+\rho_{\pi}^{j-1}\left(\rho_{\pi} \phi_{\pi}+\xi_{\pi}\right) u_{t}\right)\right) \\
& -b_{t}^{K}\left(\mu_{\pi}+\rho_{\pi}\left(\pi_{t}-\mu_{\pi}\right)+\xi_{\pi} u_{t}\right) \\
= & K \mu_{c}+\rho_{\pi} \rho_{c}\left(\pi_{t}-\mu_{\pi}+\frac{\xi_{\pi}}{\rho_{\pi}} u_{t}\right) \frac{1-\rho_{\pi}^{K}}{1-\rho_{\pi}} \\
& -b_{t}^{K}\left(\mu_{\pi}+\rho_{\pi}\left(\pi_{t}-\mu_{\pi}\right)+\xi_{\pi} u_{t}\right) .
\end{aligned}
$$

The residual $\varepsilon_{t+K}$ is known at time $t+K$ and, because it is a regression residual of the conditional regression,

$$
\begin{aligned}
E_{t-1}\left[\varepsilon_{t+K}\right] & =0 \\
\operatorname{Cov}_{t-1}\left(a_{t}^{K}+b_{t}^{K} \pi_{t}, \varepsilon_{t+K}\right) & =0 .
\end{aligned}
$$

By the law of total covariance and equations (OA.104)-(OA.105), we have

$$
\begin{aligned}
\operatorname{Cov}\left(a_{t}^{K}+b_{t}^{K} \pi_{t}, \varepsilon_{t+K}\right)= & \operatorname{Cov}\left(E_{t-1}\left[a_{t}^{K}+b_{t}^{K} \pi_{t}\right], E_{t-1}\left[\varepsilon_{t+K}\right]\right) \\
& +E\left[\operatorname{Cov}_{t-1}\left(a_{t}^{K}+b_{t}^{K} \pi_{t}, \varepsilon_{t+K}\right)\right] \\
= & 0 .
\end{aligned}
$$

Then, using (OA.106), we compute

$$
\begin{aligned}
d_{1}^{c, K} & =\frac{\operatorname{Cov}\left(\sum_{j=0}^{K-1} \Delta c_{t+1+j}, a_{t}^{K}+b_{t}^{K} \pi_{t}\right)}{\operatorname{Var}\left(a_{t}^{K}+b_{t}^{K} \pi_{t}\right)} \\
& =\frac{\operatorname{Cov}\left(a_{t}^{K}+b_{t}^{K} \pi_{t}+\varepsilon_{t+K}, a_{t}^{K}+b_{t}^{K} \pi_{t}\right)}{\operatorname{Var}\left(a_{t}^{K}+b_{t}^{K} \pi_{t}\right)} \\
& =\frac{\operatorname{Cov}\left(a_{t}^{K}+b_{t}^{K} \pi_{t}, a_{t}^{K}+b_{t}^{K} \pi_{t}\right)}{\operatorname{Var}\left(a_{t}^{K}+b_{t}^{K} \pi_{t}\right)}+\frac{\operatorname{Cov}\left(\varepsilon_{t+K}, a_{t}^{K}+b_{t}^{K} \pi_{t}\right)}{\operatorname{Var}\left(a_{t}^{K}+b_{t}^{K} \pi_{t}\right)} \\
& =\frac{\operatorname{Var}\left(a_{t}^{K}+b_{t}^{K} \pi_{t}\right)}{\operatorname{Var}\left(a_{t}^{K}+b_{t}^{K} \pi_{t}\right)}+\frac{\operatorname{Cov}\left(\varepsilon_{t+K}, a_{t}^{K}+b_{t}^{K} \pi_{t}\right)}{\operatorname{Var}\left(a_{t}^{K}+b_{t}^{K} \pi_{t}\right)} \\
& =1 .
\end{aligned}
$$


By (OA.103), (OA.107), and the law of iterated expectations,

$$
\begin{aligned}
d_{0}^{c, K} & =E\left[\sum_{j=0}^{K-1} \Delta c_{t+1+j}-d_{1}^{c, K}\left(a_{t}^{K}+b_{t}^{K} \pi_{t}\right)\right] \\
& =E\left[\sum_{j=0}^{K-1} \Delta c_{t+1+j}\right]-E\left[a_{t}^{K}\right]-E\left[b_{t}^{K} \pi_{t}\right] \\
& =E\left[\sum_{j=0}^{K-1} \Delta c_{t+1+j}\right]-E\left[E_{t}\left[\sum_{j=1}^{K} \Delta c_{t+1+j}-b_{t}^{K} \pi_{t+1}\right]\right]-E\left[b_{t}^{K} \pi_{t}\right] \\
& =E\left[\sum_{j=0}^{K-1} \Delta c_{t+1+j}\right]-E\left[\sum_{j=1}^{K} \Delta c_{t+1+j}-b_{t}^{K} \pi_{t+1}\right]-E\left[b_{t}^{K} \pi_{t}\right] \\
& =\sum_{j=0}^{K-1} E\left[\Delta c_{t+1+j}\right]-\sum_{j=1}^{K} E\left[\Delta c_{t+1+j}\right]+E\left[b_{t}^{K} \pi_{t+1}\right]-E\left[b_{t}^{K} \pi_{t}\right] .
\end{aligned}
$$

Using equations (OA.11)-(OA.13) and (OA.15), equation (OA.108) gives

$$
\begin{aligned}
d_{0}^{c, K} & =K \mu_{c}-K \mu_{c}+E\left[b_{t}^{K}\left(\pi_{t+1}-\pi_{t}\right)\right] \\
& =K \mu_{c}-K \mu_{c}+E\left[E_{t}\left[b_{t}^{K}\left(\pi_{t+1}-\pi_{t}\right)\right]\right] \\
& =E\left[b_{t}^{K}\left(E_{t}\left[\pi_{t+1}\right]-\pi_{t}\right)\right] \\
& =E\left[b_{t}^{K}\left(\mu_{\pi}+\rho_{\pi}\left(\pi_{t}-\mu_{\pi}\right)+\xi_{\pi} u_{t}-\pi_{t}\right)\right] \\
& =E\left[\left(h_{K}+\frac{\xi_{c}}{\phi_{\pi}} \varphi_{t}\right)\left(\left(\rho_{\pi}-1\right)\left(\pi_{t}-\mu_{\pi}\right)+\xi_{\pi} u_{t}\right)\right] \\
& =E\left[h_{K}+\frac{\xi_{c}}{\phi_{\pi}} \varphi_{t}\right] E\left[\left(\rho_{\pi}-1\right)\left(\pi_{t}-\mu_{\pi}\right)+\xi_{\pi} u_{t}\right] \\
& =E\left[h_{K}+\frac{\xi_{c}}{\phi_{\pi}} \varphi_{t}\right] \times 0 \\
& =0 .
\end{aligned}
$$




\subsection{Calibrated Moments in Table 12}

In this section, we compute the target moments for calibration in Table 12. Using equations (OA.11)(OA.13), the moments for consumption and inflation in the "Descriptive Statistics" part of Table 12 are

$$
\begin{aligned}
E\left[\pi_{t}\right] & =\mu_{\pi}, \\
\sigma\left(\pi_{t}\right) & =\frac{\sqrt{\left|\xi_{\pi}^{2}+2 \xi_{\pi} \rho_{\pi} \phi_{\pi}+\phi_{\pi}^{2}\right|}}{\sqrt{1-\rho_{\pi}^{2}}}, \\
A R(1) \text { of } \pi_{t} & =\rho_{\pi}+\frac{\left(1-\rho_{\pi}^{2}\right) \xi_{\pi} \phi_{\pi}}{2 \xi_{\pi} \rho_{\pi} \phi_{\pi}+\xi_{\pi}^{2}+\phi_{\pi}^{2}}, \\
E\left[\Delta c_{t}\right] & =\mu_{c}, \\
\sigma\left(\Delta c_{t}\right) & =\sqrt{\left|\sigma_{c}^{2}+\left(2 \varphi_{0} \xi_{c}+\frac{\phi_{\pi} \rho_{c}}{1-\rho_{\pi}^{2}}\right) \phi_{\pi} \rho_{c}+\left(\frac{\sigma_{w}^{2}}{1-v^{2}}+\varphi_{0}^{2}\right) \xi_{c}^{2}+\frac{\xi_{\pi} \rho_{c}^{2}\left(\xi_{\pi}+2 \phi_{\pi} \rho_{\pi}\right)}{1-\rho_{\pi}^{2}}\right|} \\
A R(1) \text { of } \Delta c_{t} & =\frac{\rho_{c}\left(1-\nu^{2}\right)\left(\phi_{\pi} \rho_{\pi}+\xi_{\pi}\right)\left(\rho_{c}\left(\phi_{\pi}+\xi_{\pi} \rho_{\pi}\right)+\xi_{c} \varphi_{0}\left(1-\rho_{\pi}^{2}\right)\right)}{\left(1-\rho_{\pi}^{2}\right)\left[\left(1-\nu^{2}\right)\left(\rho_{c}^{2} \sigma^{2}\left(\pi_{t}\right)+\left(\varphi_{0} \xi_{c}\left(\varphi_{0} \xi_{c}+2 \phi_{\pi} \rho_{c}\right)+\sigma_{c}^{2}\right)\right)+\xi_{c}^{2} \sigma_{w}^{2}\right]}, \\
E\left[u_{\Pi, t}\right] & =0, \\
\sigma\left(u_{\Pi, t}\right) & =\left|\phi_{\pi}\right| \\
A R(1) \text { of } u_{\Pi, t} & =0, \\
\operatorname{corr}\left(\pi_{t}, u_{\Pi, t}\right) & =\frac{\left|\phi_{\pi}\right|}{\sigma\left(\pi_{t}\right)}, \\
\operatorname{corr}\left(\pi_{t}, \Delta c_{t}\right) & =\frac{\xi_{\pi}+\rho_{\pi} \phi_{\pi}}{\sigma\left(\pi_{t}\right) \sigma\left(\Delta c_{t}\right)}\left(\varphi_{0} \xi_{c}+\frac{\left(\phi_{\pi}+\xi_{\pi} \rho_{\pi}\right) \rho_{c}}{1-\rho_{\pi}^{2}}\right) \\
\operatorname{corr}\left(\Delta c_{t}, u_{\Pi, t}\right) & =0 .
\end{aligned}
$$

The moments for the "Predictve Regressions of Consumption Growth on Inflation" part of Table 12 are given by equations (OA.102), (OA.101), (OA.109), (OA.107) and by

$$
\begin{aligned}
A R(1) \text { of } N R C_{t}^{C} & =v \\
\sigma\left(N R C_{t}^{C}\right) & =\frac{1}{\sqrt{12}} \frac{1}{\sqrt{1-v^{2}}}\left|\frac{\xi_{c} \sigma_{w}}{\phi_{\pi}}\right|,
\end{aligned}
$$

which follow from using equations (OA.13), (OA.15)-(OA.17). We now compute moments for assets $i=$ $H, L, M K T$, where $H$ and $L$ represent in the model the highest and lowest inflation beta portfolios that we constructed in the data, and $M K T$ is the market portfolio. Taking unconditional expectations in equation (OA.52) gives mean returns for asset $i$

$$
E\left[r_{i, t}\right]=\kappa_{i, 0}+\mu_{i}-D_{i, 0}\left(1-\kappa_{i, 1}\right)
$$


Plugging equation (OA.52) into (OA.51) and computing the unconditional variance gives the variance of returns for asset $i$, denoted $\sigma^{2}\left(r_{i, t}\right)$

$$
\begin{aligned}
\sigma^{2}\left(r_{i, t}\right)= & 2 \varphi_{0} \phi_{\pi} D_{i, 1} D_{i, 2}\left(1-\rho_{\pi} \kappa_{1,1}+\kappa_{1,1}^{2}\right)+\varphi_{0}\left(\xi_{1} \varphi_{0}+\rho_{1} \phi_{\pi}\right)\left(\xi_{1}-2 D_{i, 2}\right) \\
& +\varphi_{0}^{2}\left(\kappa_{1,1}^{2}+1\right) D_{i, 2}^{2}+\xi_{1} \varphi_{0} \rho_{1} \phi_{\pi}+\sigma_{1}^{2} \\
& -\frac{\phi_{\pi}}{1-\rho_{\pi}^{2}}\left(2\left(1-\rho_{\pi} \kappa_{1,1}\right)\left(\rho_{1} \phi_{\pi}+\left(1-\rho_{\pi}^{2}\right) \xi_{1} \varphi_{0}\right) D\right) \\
& +\frac{\phi_{\pi}}{1-\rho_{\pi}^{2}}\left(\phi_{\pi} D_{i, 1}^{2}\left(\kappa_{1,1}^{2}-2 \rho_{\pi} \kappa_{1,1}+1\right)-\rho_{1}^{2} \phi_{\pi}\right) \\
& +\frac{\sigma_{w}^{2}}{1-v^{2}}\left(\left(2 \varphi_{0} D_{i, 5}+D_{1,4}\right)^{2}\left(\kappa_{1,1}^{2}-2 v \kappa_{1,1}+1\right)+\left(\xi_{1}-D_{i, 2}\right)^{2}+D_{i, 2}^{2} \kappa_{1,1}^{2}\right) \\
& +\frac{2 \sigma_{w}^{4}}{\left(v^{2}-1\right)^{2}}\left(\kappa_{1,1}^{2}-2 v^{2} \kappa_{1,1}+1\right) D_{i, 5}^{2} \\
& +\frac{1}{1-\rho_{\pi}^{2}}\left(\left(\rho_{1}-D_{i, 1}\left(1-\rho_{\pi} \kappa_{i, 1}\right)\right)^{2}\right) \xi_{\pi}^{2}+\xi_{\pi}^{2} \kappa_{i, 1}^{2} D_{i, 1}^{2} \\
& +\frac{2 \phi_{\pi}}{1-\rho_{\pi}^{2}}\left(\left(\rho_{\pi}-\kappa_{1,1}\right)\left(1-\rho_{\pi} \kappa_{1,1}\right) D_{i, 1}^{2}+\left(\left(\kappa_{1,1} \rho_{\pi}^{2}-2 \rho_{\pi}+\kappa_{1,1}\right) D_{i, 1}+\rho_{1} \rho_{\pi}\right) \rho_{1}\right) \xi_{\pi} \\
& -2 \kappa_{1,1} D_{1,1}\left(-\varphi_{0}\left(\xi_{1}-D_{i, 2}\right)+D_{1,3}\right) \xi_{\pi}+\left(\kappa_{1,1}^{2}+1\right) D_{i, 3}^{2} \\
& +2\left(\left(\left(\kappa_{1,1}^{2}+1\right) D_{i, 2}-\xi_{1}\right) \varphi_{0}+\phi_{\pi}\left(\left(\kappa_{1,1}^{2}-\rho_{\pi} \kappa_{1,1}+1\right) D_{i, 1}-\rho_{1}\right)\right) D_{i, 3} .
\end{aligned}
$$

The standard deviation of returns is simply $\sigma\left(r_{i, t}\right)$. We find the mean inflation beta for asset $i, E\left[\beta_{\Pi, i, t}\right]$, and its standard deviation, $\sigma\left(\beta_{\Pi, i, t}\right)$, by taking the mean and standard deviation in equation (OA.53) and using equation (OA.57)

$$
\begin{aligned}
E\left[\beta_{\Pi, i, t}\right] & =\frac{\kappa_{1, i}}{\left|\phi_{\pi}\right|}\left(\varphi_{0} D_{i, 2}+\phi_{\pi} D_{i, 1}+D_{i, 3}\right), \\
\sigma\left(\beta_{\Pi, i, t}\right) & =\frac{\kappa_{i, 1}}{\sqrt{1-v^{2}}}\left|\frac{\sigma_{w} D_{i, 2}}{\phi_{\pi}}\right| .
\end{aligned}
$$

Also using equation (OA.53), the correlation in inflation betas for two assets $i, j$ is

$$
\operatorname{corr}\left(\beta_{\Pi, i, t}, \beta_{\Pi, j, t}\right)=\operatorname{sign}\left(D_{i, 2} D_{j, 2}\right) .
$$

Denote the high-minus-low inflation portfolio by $i=I P^{H L}$, with returns defined by

$$
r_{I P^{H L}, t} \equiv r_{H, t}-r_{L, t}
$$


Eqs. (OA.52) and (OA.51) give

$$
\begin{aligned}
r_{I P^{H L}, t}= & r_{H, t+1}-r_{L, t+1} \\
= & E_{t}\left[r_{I P^{H L}, t+1}\right]+\left(\beta_{u, H t}-\beta_{u, L t}\right) u_{t+1} \\
& +\left(\beta_{w, H}-\beta_{w, L}\right) w_{t+1}+\left(\beta_{2 w, H}-\beta_{2 w, L}\right)\left(\frac{w_{t+1}^{2}-1}{\sqrt{2}}\right) \\
& +\left(\beta_{\eta, H}-\beta_{\eta, L}\right) \eta_{t+1},
\end{aligned}
$$

where

$$
\begin{aligned}
E_{t}\left[r_{I P^{H L}, t+1}\right]= & E_{t}\left[r_{H, t+1}\right]-E_{t}\left[r_{L, t+1}\right] \\
= & \kappa_{H, 0}+\mu_{H}-D_{H, 0}\left(1-\kappa_{H, 1}\right) \\
& -\left(\kappa_{L, 0}+\mu_{L}-D_{L, 0}\left(1-\kappa_{L, 1}\right)\right) \\
& +\left(\rho_{H}-D_{H, 1}\left(1-\kappa_{H, 1} \rho_{\pi}\right)-\left(\rho_{L}-D_{L, 1}\left(1-\kappa_{L, 1} \rho_{\pi}\right)\right)\right)\left(\pi_{t}-\mu_{\pi}\right) \\
& -\left(D_{H, 3}-\xi_{\pi} \kappa_{H, 1} D_{H, 1}-\left(D_{L, 3}-\xi_{\pi} \kappa_{L, 1} D_{L, 1}\right)\right) u_{t} \\
& +\left(\xi_{H}-D_{H, 2}-\left(\xi_{L}-D_{L, 2}\right)\right) \varphi_{t-1} u_{t} \\
& +\left(2 D_{H, 5} v \kappa_{H, 1} \varphi_{0}(1-v)-D_{H, 4}\left(1-v \kappa_{H, 1}\right)\right)\left(\varphi_{t}-\varphi_{0}\right) \\
& -\left(2 D_{L, 5} v \kappa_{L, 1} \varphi_{0}(1-v)-D_{L, 4}\left(1-v \kappa_{L, 1}\right)\right)\left(\varphi_{t}-\varphi_{0}\right) \\
& -\left(D_{H, 5}\left(1-v^{2} \kappa_{H, 1}\right)-\left(D_{L, 5}\left(1-v^{2} \kappa_{L, 1}\right)\right)\right)\left(\varphi_{t}^{2}-E\left[\varphi_{t}^{2}\right]\right) .
\end{aligned}
$$

Eq. (OA.53) can be used together with equations (OA.57) and (OA.110) to get

$$
\begin{aligned}
\beta_{\Pi, I P^{H L}, t} & =\frac{\operatorname{Cov}\left(r_{I P^{H L}, t}, u_{\Pi, t}\right)}{\operatorname{Var}\left(u_{\Pi, t}\right)} \\
& =\frac{1}{\left|\phi_{\pi}\right|} \frac{\operatorname{Cov}\left(r_{I P^{H L}, t}, u_{t}\right)}{\operatorname{Var}\left(u_{t}\right)} \\
& =\frac{1}{\left|\phi_{\pi}\right|} \operatorname{Cov}\left(r_{H, t}-r_{L, t}, u_{\Pi, t}\right) \\
& =\frac{1}{\left|\phi_{\pi}\right|} \operatorname{Cov}\left(r_{H, t}, u_{u, t}\right)-\frac{1}{\left|\phi_{\pi}\right|} \operatorname{Cov}\left(r_{L, t}, u_{u, t}\right) \\
& =\frac{1}{\left|\phi_{\pi}\right|} \beta_{u, H, t}-\frac{1}{\left|\phi_{\pi}\right|} \beta_{u, L, t} \\
& =\beta_{\Pi, H, t}-\beta_{\Pi, L, t} \\
& =\frac{\kappa_{H, 1}}{\left|\phi_{\pi}\right|}\left(\phi_{\pi} D_{H, 1}+D_{H, 2} \varphi_{t}+D_{H, 3}\right) \\
& -\frac{\kappa_{L, 1}}{\left|\phi_{\pi}\right|}\left(\phi_{\pi} D_{L, 1}+D_{L, 2} \varphi_{t}+D_{L, 3}\right) .
\end{aligned}
$$


The standard deviation of the inflation beta of $I P^{H L}$ can be found using equation (OA.114)

$$
\begin{aligned}
\sigma\left(\beta_{u, I P^{H L}, t}\right) & =\sigma\left(\beta_{u, 1 t}-\beta_{u, 2 t}\right) \\
& =\frac{1}{\sqrt{1-v^{2}}}\left|\frac{\sigma_{w}\left(D_{1,2} \kappa_{1,1}-D_{2,2} \kappa_{2,1}\right)}{\phi_{\pi}}\right| .
\end{aligned}
$$

The coefficient $L^{N R C}$ of a regression of the returns of $I P_{t}^{H L}$ on the standardized nominal-real covariance is

$$
\begin{aligned}
L_{N R C, I P_{t}^{H L}} & =\frac{\operatorname{cov}\left(r_{I P^{H L}, t}, \tilde{\theta}_{t}^{(12)}\right)}{\operatorname{var}\left(\tilde{\theta}_{t}^{(12)}\right)} \\
& =\operatorname{cov}\left(r_{I P^{H L}, t}, \tilde{\theta}_{t}^{(12)}\right)
\end{aligned}
$$

where $\tilde{\theta}_{t}^{(12)}$ is defined in equation (OA.86). Using equations (OA.51), (OA.52), (OA.86), (OA.110) we get

$$
\begin{aligned}
L_{N R C, I P_{t}^{H L}}= & \operatorname{cov}\left(r_{I P^{H L}, t}, \tilde{\theta}_{t}^{(12)}\right) \\
= & -\left(1-v \kappa_{1,1}\right) \frac{\operatorname{sign}\left(\phi_{\pi}\right) \operatorname{sign}\left(\xi_{c}\right)\left|\sigma_{w}\right|}{\sqrt{1-v^{2}}}\left(2 \varphi_{0} D_{1,5}+D_{1,4}\right) \\
& +\left(1-v \kappa_{2,1}\right) \frac{\operatorname{sign}\left(\phi_{\pi}\right) \operatorname{sign}\left(\xi_{c}\right)\left|\sigma_{w}\right|}{\sqrt{1-v^{2}}}\left(2 \varphi_{0} D_{2,5}+D_{2,4}\right)
\end{aligned}
$$

Last, the regression coefficient $L_{\beta_{\Pi} \times N R C, m}$ is given in equation (OA.97). The numbers reported in Table 12 are annualized and in percentage points. More precisely, the numbers reported in Table 12 are obtained by using the formulas above and then by multiplying $E\left[\pi_{t}\right], E\left[u_{\Pi, t}\right], E\left[\Delta c_{t}\right], E\left[r_{i, t}\right], L_{N R C, I P_{t}^{H L}}$ by $100 \times 12$; $\sigma\left(\pi_{t}\right), \sigma\left(u_{\Pi, t}\right), \sigma\left(\Delta c_{t}\right), \sigma\left(r_{i, t}\right)$ by $100 \times \sqrt{12}$; all correlations, $A R(1)$ coefficients, and $R^{2}$ by 100 ; the coefficient $L_{\beta_{\Pi} \times N R C, m}$ by 100 (no need to multiply by 12 because it was obtained using cumulative returns over 12 months). 
3 Robustness Checks 


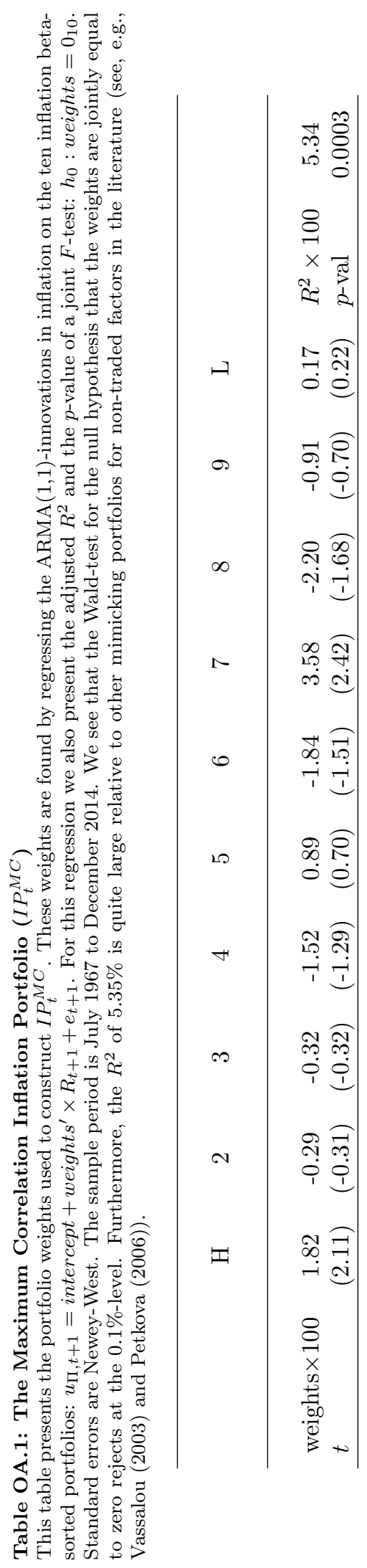




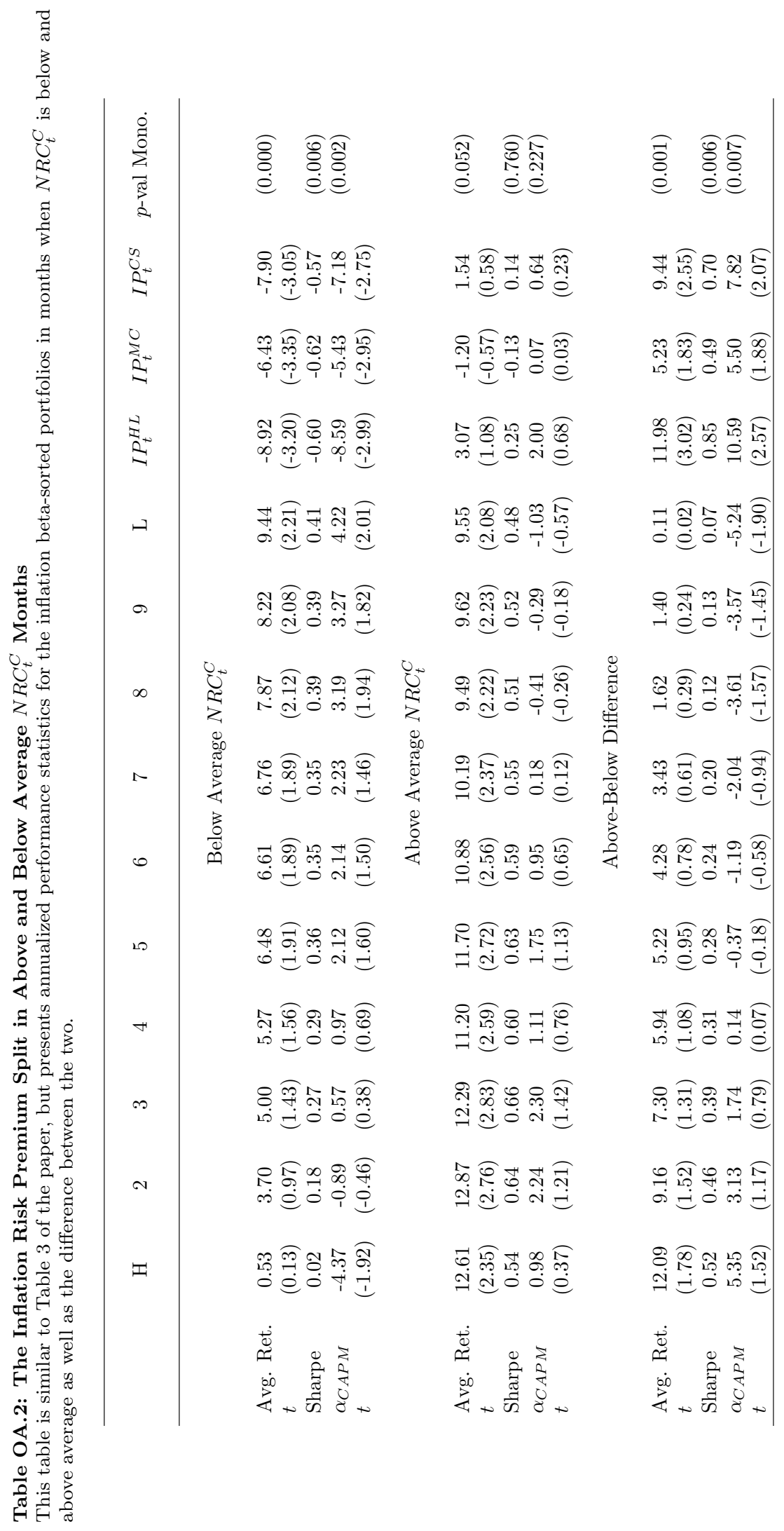




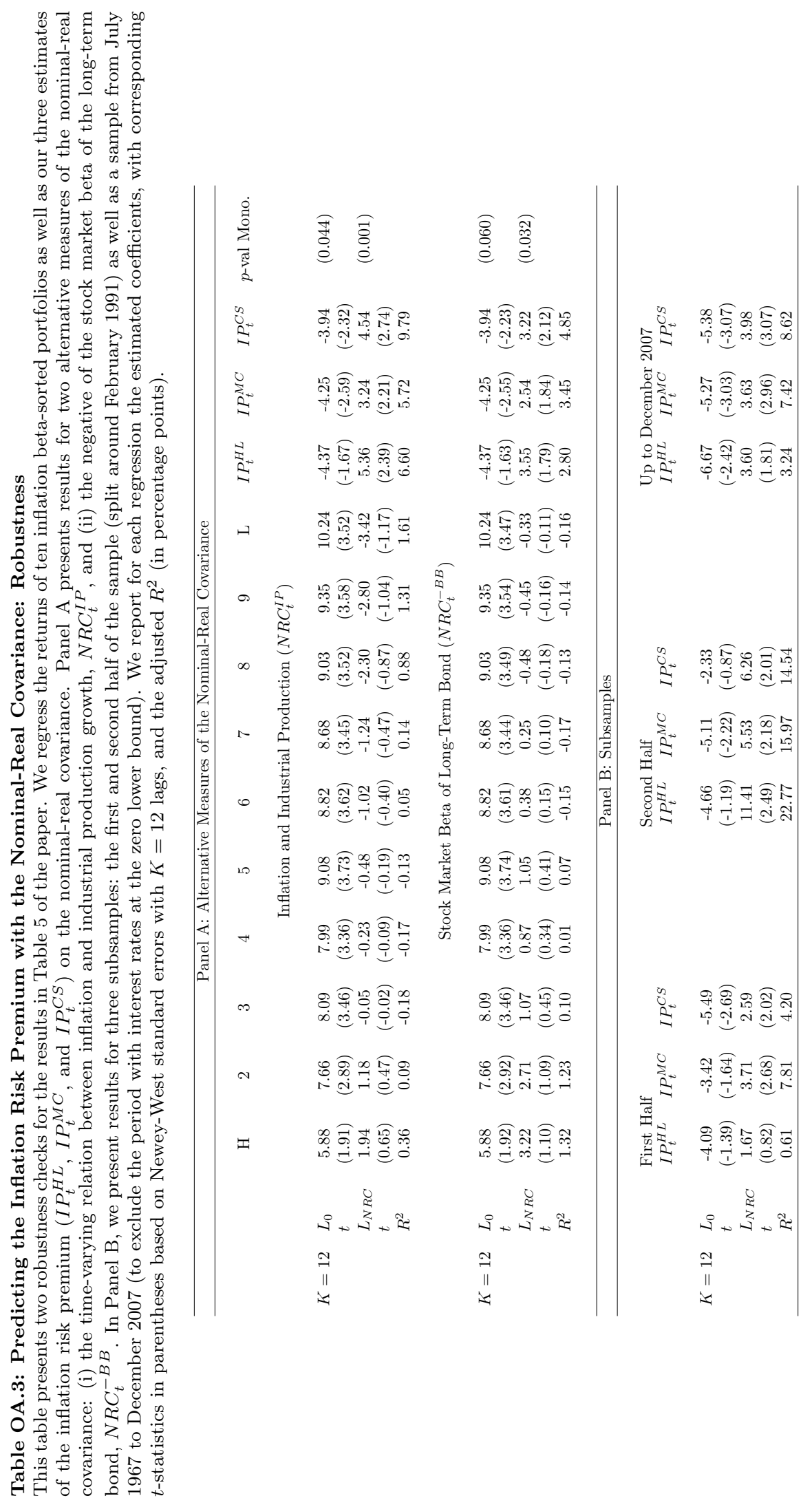


Table OA.4: Time-Varying Inflation Risk Premia in Pooled Regressions at Shorter Horizons

This table is similar to Panel A of Table 6 except that results are presented for the one- and three-month horizon. We present estimated coefficients (with asymptotic Driscoll and Kraay (1998) standard errors in parentheses) from various pooled predictive regressions of returns on the inflation beta-sorted decile portfolios on their inflation beta $\left(\beta_{\Pi, p, t}\right)$, the nominal-real covariance $\left(N R C_{t}^{C}\right)$, and an interaction. In Model [1], we set $L_{\beta_{\Pi} \times N R C}=0$, whereas Model [2] estimates all three coefficients freely. Models [3] to [5] in Panel A analyze which components of inflation betas interact with the nominal-real covariance. Model [3] replaces the interaction term, $\beta_{\Pi, p, t} \times N R C_{t}^{C}$, with the interaction between the nominal-real covariance and the portfoliospecific component of inflation betas, $\widehat{\beta_{\Pi, p}}=T^{-1} \sum_{t=1}^{T} \beta_{\Pi, p, t}$. Model [4] replaces the interaction term, $\beta_{\Pi, p, t} \times N R C_{t}^{C}$, with the interaction between the nominal-real covariance and the time-specific component of inflation betas, $\widehat{\beta_{\Pi, t}}=10^{-1} \sum_{p=1}^{10} \beta_{\Pi, p, t}$. Model [5] includes both component-wise interaction terms. To accommodate interpretation, $N R C_{t}^{C}$ is standardized in the time series to have mean zero and standard deviation equal to one, whereas $\beta_{\Pi, p, t}$ is demeaned in the pool. The sample period is July 1967 to December 2014.

\begin{tabular}{lccccccccccc}
\hline \multicolumn{1}{c}{ Horizon $K=1$ Month } & \multicolumn{1}{c}{ Horizon $K=3$ Months } \\
Model & {$[1]$} & {$[2]$} & {$[3]$} & {$[4]$} & {$[5]$} & {$[1]$} & {$[2]$} & {$[3]$} & {$[4]$} & {$[5]$} \\
& & & & & & & & & & & \\
& & & & & & & & & & & \\
$L_{0}$ & 7.97 & 4.76 & 7.97 & 4.55 & 4.57 & 8.29 & 4.82 & 8.29 & 4.63 & 4.66 \\
$t$ & $(2.69)$ & $(1.36)$ & $(2.69)$ & $(1.24)$ & $(1.25)$ & $(3.09)$ & $(1.66)$ & $(3.10)$ & $(1.54)$ & $(1.55)$ \\
$L_{\beta_{\Pi}}$ & -0.22 & -1.12 & -0.19 & -1.24 & -1.20 & 0.11 & -0.88 & 0.15 & -1.00 & -0.95 \\
$t$ & $(-0.19)$ & $(-0.87)$ & $(-0.15)$ & $(-0.92)$ & $(-0.89)$ & $(0.11)$ & $(-0.92)$ & $(0.15)$ & $(-1.01)$ & $(-0.96)$ \\
$L_{N R C}$ & -0.28 & -0.96 & -0.34 & -0.91 & -0.96 & -1.12 & -1.89 & -1.19 & -1.82 & -1.88 \\
$t$ & $(-0.07)$ & $(-0.26)$ & $(-0.09)$ & $(-0.24)$ & $(-0.26)$ & $(-0.33)$ & $(-0.57)$ & $(-0.35)$ & $(-0.56)$ & $(-0.57)$ \\
$L_{\beta_{\Pi} \times N R C}$ & & 2.07 & & & & & 2.27 & & & \\
$t$ & & $(1.88)$ & & & & & $(2.53)$ & & & 1.95 \\
$L_{\beta_{\Pi, p} \times N R C}$ & & & 1.87 & & 1.64 & & & 2.21 & & $(2.43)$ \\
$t$ & & & $(2.19)$ & & $(2.01)$ & & & $(2.63)$ & & \\
$L_{\beta_{\Pi, t} \times N R C}$ & & & & 2.21 & 2.19 & & & & 2.39 & 2.37 \\
$t$ & & & & $(1.80)$ & $(1.79)$ & & & & $(2.41)$ & $(2.39)$ \\
$R^{2}$ & 0.02 & 0.69 & 0.06 & 0.68 & 0.71 & 0.05 & 2.08 & 0.21 & 1.99 & 2.11 \\
\hline
\end{tabular}


Table OA.5: The Nominal-Real Covariance and the Average Risk Premium

This table is similar to Table 7 of the paper and presents coefficient estimates from time-series predictive regressions for the equal-weighted average return over the ten decile portfolios on the inflation beta of this average portfolio $\left(\beta_{\Pi, \text { avg,t }}\right)$, the nominalreal covariance $\left(N R C_{t}^{C}\right)$, and an interaction term. We present this regression for the twelve-month horizon and standard errors are Newey-West(12). To accommodate interpretation, $N R C_{t}^{C}$ is standardized and $\beta_{\Pi, a v g, t}$ is demeaned. The sample period is July 1967 to December 2014.

\begin{tabular}{lcc}
\hline & {$[1]$} & {$[2]$} \\
& & \\
\hline$L_{0, \text { avg }}$ & 8.48 & 5.06 \\
$t$ & $(3.47)$ & $(1.98)$ \\
$L_{\beta_{\Pi}, \text { avg }}$ & 0.81 & -0.45 \\
$t$ & $(0.75)$ & $(-0.51)$ \\
$L_{N R C, \text { avg }}$ & -2.53 & -3.04 \\
$t$ & $(-0.76)$ & $(-1.12)$ \\
$L_{\beta_{\Pi} \times N R C, \text { avg }}$ & & 2.38 \\
$t$ & & $(3.31)$ \\
$R^{2}$ & 0.90 & 8.44 \\
\hline
\end{tabular}

Table OA.6: Time-Varying Inflation Risk Premia in Pooled Regressions Controlling for Market Beta

This table is similar to Panel A of Table 6 except that we now also control for each portfolio's market beta (estimated by regressing in month $t$ each portfolio's returns on the CRSP value-weighted market portfolio over an expanding window of historical returns, similar to the way inflation betas are measured). We present estimated coefficients (with asymptotic Driscoll and Kraay (1998) standard errors in parentheses) from various pooled predictive regressions. In Model [1], we regress returns on the inflation beta-sorted decile portfolios on their market beta $\left(\beta_{M K T, p, t}\right)$, the nominal-real covariance $\left(N R C_{t}^{C}\right)$, and their interaction. In Model [2], we add inflation betas $\left(\beta_{\Pi, p, t}\right)$ and their interaction with the nominal-real covariance. In Model [3], we decompose the inflation beta for the interaction with the nominal-real covariance in the portfolio- and time-specific components: $\widehat{\beta_{\Pi, p}}=T^{-1} \sum_{t=1}^{T} \beta_{\Pi, p, t}$ and $\widehat{\beta_{\Pi, t}}=10^{-1} \sum_{p=1}^{10} \beta_{\Pi, p, t}$. To accommodate interpretation, $N R C_{t}^{C}$ is standardized in the time series to have mean zero and standard deviation equal to one, whereas $\beta_{\Pi, p, t}$ is demeaned in the pool. The sample period is July 1967 to December 2014 and we focus on the twelve-month horizon.

\begin{tabular}{lccc}
\hline Model & {$[1]$} & {$[2]$} & {$[3]$} \\
& & & \\
\hline & & & \\
$L_{0}$ & 6.31 & 18.22 & 18.46 \\
& $(0.60)$ & $(1.80)$ & $(1.52)$ \\
$L_{\beta_{M K T}}$ & 2.02 & -12.00 & -12.18 \\
$L_{\beta_{M K T} \times N R C}$ & $(0.20)$ & $(-1.19)$ & $(-1.05)$ \\
$L_{\beta_{\Pi}}$ & $(-0.46$ & 3.71 & 3.49 \\
& & $(0.42)$ & $(0.39)$ \\
$L_{N R C}$ & 6.10 & -0.82 & -0.82 \\
& $(0.60)$ & $(-0.63)$ & $(-0.94)$ \\
$L_{\beta_{\Pi} \times N R C}$ & & 2.61 & $(-0.63)$ \\
$L_{\beta_{\Pi, p} \times N R C}$ & & $(3.69)$ & \\
$L_{\beta_{\Pi, t} \times N R C}$ & & & 3.28 \\
$R^{2}$ & & & $2.34)$ \\
\hline
\end{tabular}


Table OA.7: Industry Composition of Inflation Beta-Sorted Portfolios

This table presents the industry composition of our portfolios. For this exercise, we use the classification into 48 industries from Kenneth French's web site. In each sample month, we calculate for each industry the number of stocks that have inflation beta below or above the median inflation beta in the full cross-section. We then translate this number to the fraction of an industry's total market capitalization with below or above median inflation beta. In the first three columns we report results for the top 10 inflation hedgers, which are those industries with on average the largest fraction of market cap in the above median inflation beta portfolio. For these industries we report the average allocation to the above median portfolio (" $\%$ of market cap $\left(\beta_{\Pi, i, t}>\right.$ median)") as well as the fraction of total sample months in which the allocation to the above median portfolio is larger than $50 \%$ ("\% of months"). Consistent with the evidence in Table 8, we find oil, gold, and mining among the best conditional inflation hedgers throughout our sample. Over $64 \%$ on average of the market capitalization in these industries has an above median inflation beta, and in over $70 \%$ of the months in our sample, over half of the market capitalization of these same industries is allocated to the above median inflation beta group. It is intuitive that we find utilities among the best conditional inflation hedgers as well. The next three columns present analogous evidence for the top 10 worst inflation hedgers, which are those industries with on average the largest fraction of market cap in the below median inflation beta portfolio. Among the worst inflation hedgers we find meals, clothes, and textiles (as in Table 8), but also banks and insurance.

Top 10 Best Inflation Hedgers

Industry

$\%$ of market cap

$\left(\beta_{\Pi, i, t}>\right.$ median $)$
Top 10 Worst Inflation Hedgers

$$
\begin{aligned}
& \% \text { of market cap } \quad \% \text { of months } \\
& \left(\beta_{\Pi, i, t} \leq \text { median }\right)
\end{aligned}
$$

\begin{tabular}{lllllll}
\hline & & & & & & \\
1 & Oil & 0.77 & 0.80 & Other & 0.82 & 0.94 \\
2 & Gold & 0.73 & 0.76 & Books & 0.77 & 0.95 \\
3 & Utilities & 0.66 & 0.68 & Aerospace & 0.76 & 0.89 \\
4 & Mines & 0.64 & 0.70 & Banks & 0.74 & 0.84 \\
5 & Agriculture & 0.62 & 0.71 & Insurance & 0.74 & 0.88 \\
6 & Paper & 0.61 & 0.66 & Meals & 0.73 & 0.82 \\
7 & Computers & 0.60 & 0.67 & Lab Equipment & 0.72 & 0.82 \\
8 & Steel & 0.58 & 0.59 & Clothes & 0.71 & 0.79 \\
9 & Smoke & 0.58 & 0.66 & Textiles & 0.71 & 0.82 \\
10 & Food & 0.57 & 0.59 & Personal Services & 0.70 & 0.85 \\
\hline
\end{tabular}


Table OA.8: The timing premium in our model of the nominal-real covariance

This table shows the size of the timing premium for our model under three parameter configurations. The first column shows the timing premium under our baseline calibration found in Table 11. We display only the parameters that change across columns, the rest of the parameters are held fixed at the values shown in Table 11. The second column increases the persistence of inflation $\left(\rho_{\pi}\right)$ and the degree of predictability that inflation has on future consumption $\left(\rho_{c}\right)$ so that inflation mimics long-run risk as calibrated in Bansal et al. (2012) (in Table, the persistence of long-run risk is denoted by $a$ and the degree of predictability that long-run risk has on future consumption is always -1). In the third column, we modify our baseline calibration by postulating a nominal-real covariance that is much more persistent (higher $\nu$ ) and volatile (higher $\sigma_{w}$ ).

\begin{tabular}{lccc} 
& Baseline & $\begin{array}{c}\text { Inflation has long- } \\
\text { run risk persistence }\end{array}$ & $\begin{array}{c}\text { More volatile and } \\
\text { persistent } \varphi_{t}\end{array}$ \\
\hline$\rho_{\pi}$ & & & \\
$\rho_{c}$ & 0.799 & 0.979 & 0.799 \\
$\nu$ & -0.052 & -1 & -0.052 \\
$\sigma_{w}$ & 0.9963 & 0.9963 & 0.999 \\
Timing premium & 0.0029 & 0.0029 & 0.02 \\
\hline
\end{tabular}


Table OA.9: The timing premium in the long-run risk model

This table shows the size of the timing premium in the long-run risk model of Bansal et al. (2012) under three parameter configurations. The first two columns reproduce the results in Table 1 of Epstein et al. (2014) and show the timing premium in versions of the long-run risk model with and without stochastic volatility. In the third column, we compute the timing premium in the long-run risk model with stochastic volatility but replacing the coefficient of relative risk aversion (RRA) and the elasticity of intertemporal substitution (EIS) by the values of our main calibration in Table 11.

\begin{tabular}{lccc} 
Parameter & LLR no stoch. vol. & LLR with stoch. vol. & Higher RRA and EIS \\
\hline & & & \\
$\sigma$ & 0.0078 & 0.0078 & 0.0078 \\
$\phi$ & 0.044 & 0.044 & 0.044 \\
$a$ & 0.9790 & 0.9790 & 0.9790 \\
$\sigma_{w}$ & 0 & $0.23 \times 10^{-5}$ & $0.23 \times 10^{-5}$ \\
$\nu$ & 0 & 0.987 & 0.987 \\
$\beta$ & 0.998 & 0.998 & 0.998 \\
RRA & 7.5 or 10 & 7.5 or 10 & 15 \\
EIS & 1.5 & 1.5 & 2 \\
Timing premium & $23 \%$ or $29 \%$ & $24 \%$ or $31 \%$ & $44.0 \%$ \\
\hline
\end{tabular}




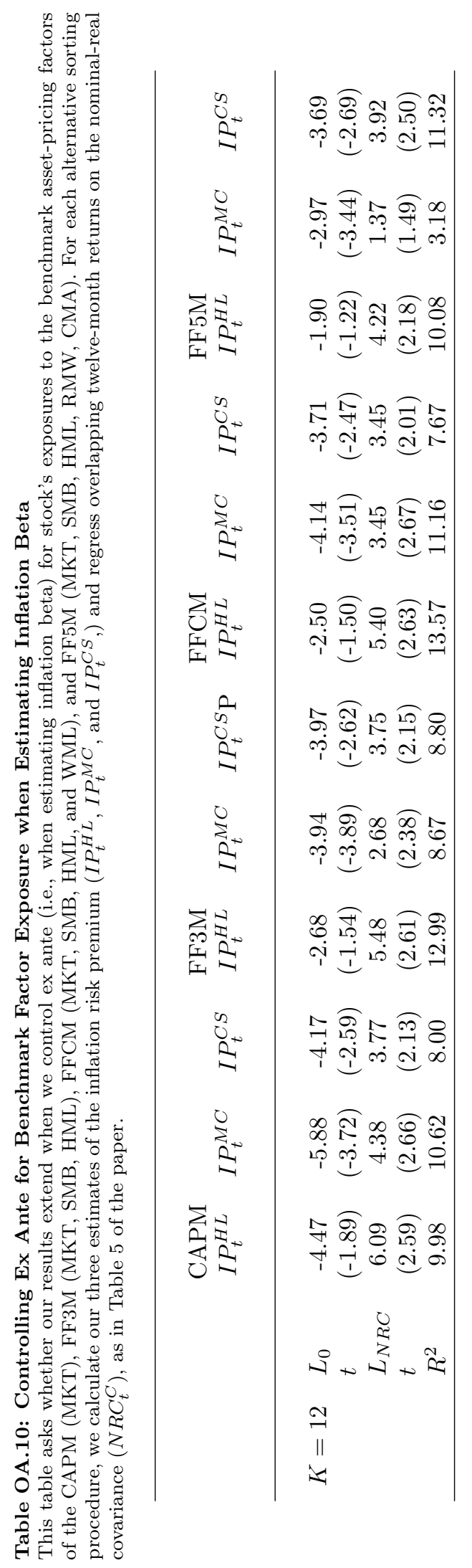


Table OA.11: Controlling Ex Post for Benchmark Factor Exposure when Predicting the Inflation Risk Premium This table asks whether our conclusions on the time-varying inflation risk premium extend when we control ex post for exposure in our estimates of the inflation risk premium to the benchmark asset pricing factors. For this exercise, we regress the returns of $I P_{t}^{H L}, I P_{t}^{M C}$, and $I P_{t}^{C S}$ on the nominal-real covariance $\left(N R C_{t}^{C}\right)$ as well as on contemporaneous exposure to the factors in the CAPM, FF3M, FFCM, and FF5M. We focus on the annual horizon $K=12$ and calculate overlapping twelve-month compounded returns on both the left- and right-hand side. We presents for each regression the estimated coefficients and the adjusted $R^{2}$ (in percentage points). To conserve space, we present $t$-statistics in parentheses (based on Newey-West standard errors with $K$ lags) only for the intercept and the coefficient on $N R C_{t}^{C}\left(L_{0}\right.$ and $\left.L_{N R C}\right)$.

\begin{tabular}{|c|c|c|c|c|c|c|c|c|c|c|c|c|c|}
\hline$R_{t+1: t+K}$ & & $\begin{array}{l}\text { CAPM } \\
I P_{t}^{H L}\end{array}$ & $I P_{t}^{M C}$ & $I P_{t}^{C S}$ & $\begin{array}{l}\text { FF3M } \\
I P_{t}^{H L}\end{array}$ & $I P_{t}^{M C}$ & $I P_{t}^{C S}$ & $\begin{array}{l}\text { FFCM } \\
I P_{t}^{H L}\end{array}$ & $I P_{t}^{M C}$ & $I P_{t}^{C S}$ & $\begin{array}{l}\text { FF5M } \\
I P_{t}^{H L}\end{array}$ & $I P_{t}^{M C}$ & $I P_{t}^{C S}$ \\
\hline \multirow[t]{10}{*}{$K=12$} & $L_{0}$ & $\begin{array}{l}-4.98 \\
(-162)\end{array}$ & $\begin{array}{l}-3.56 \\
(-186)\end{array}$ & -4.30 & $\begin{array}{l}-3.85 \\
(-174\end{array}$ & $\begin{array}{l}-2.31 \\
(-1883\end{array}$ & $\begin{array}{l}-3.09 \\
(-204\end{array}$ & 0.48 & $\begin{array}{l}-0.98 \\
(-0.68)\end{array}$ & $\begin{array}{l}-1.43 \\
(-0.91)\end{array}$ & 1.28 & 0.85 & 0.32 \\
\hline & $L_{N R C}$ & $\begin{array}{c}-1.02) \\
5.46\end{array}$ & $\begin{array}{c}-1.00 \\
3.65\end{array}$ & 4.56 & 4.96 & $\begin{array}{c}-1.00) \\
3.08\end{array}$ & $\begin{array}{c}-2.04) \\
4.01\end{array}$ & 3.45 & $\begin{array}{c}-0.00) \\
2.62\end{array}$ & $\begin{array}{c}-0.71 \\
3.43\end{array}$ & 4.14 & 2.57 & 3.46 \\
\hline & $t$ & $(2.21)$ & $(2.52)$ & $(2.76)$ & $(2.07)$ & $(2.25)$ & $(2.48)$ & (1.64) & $(2.09)$ & $(2.25)$ & (1.66) & (1.78) & $(2.08)$ \\
\hline & $\beta_{M K T}$ & 0.10 & -0.11 & 0.06 & 0.16 & -0.09 & 0.08 & 0.09 & -0.11 & 0.05 & -0.01 & -0.20 & -0.03 \\
\hline & $\beta_{S M B}$ & & & & -0.52 & -0.31 & -0.35 & -0.54 & -0.32 & -0.36 & -0.58 & -0.35 & -0.40 \\
\hline & $\beta_{H M L}$ & & & & -0.07 & -0.12 & -0.11 & -0.20 & -0.16 & -0.16 & 0.32 & 0.11 & 0.11 \\
\hline & $\beta_{W M L}$ & & & & & & & -0.35 & -0.11 & -0.14 & & & \\
\hline & $\beta_{R M W}$ & & & & & & & & & & -0.87 & -0.55 & -0.68 \\
\hline & $\beta_{C M A}$ & & & & & & & & & & -0.52 & -0.30 & -0.23 \\
\hline & & 7.19 & 9.57 & 10.01 & 15.56 & 17.66 & 18.56 & 22.05 & 19.00 & 20.42 & 35.63 & 36.27 & 41.53 \\
\hline
\end{tabular}

Table OA.12: Controlling for Benchmark Predictors when Predicting the Inflation Risk Premium

This table asks whether our conclusions on the time-varying inflation risk premium extend when we control for benchmark predictors. For this exercise, we regress the returns of $I P_{t}^{H L}, I P_{t}^{M C}$, and $I P_{t}^{C S}$ on the nominal-real covariance $\left(N R C_{t}^{C}\right)$ controlling for either the dividend yield (DY), default spread (DS), and term spread (TS) or the consumption-wealth ratio (CAY). All control variables are standardized. We present the coefficient estimates and, to conserve space, report $t$-statistics in parentheses (based on Newey-West standard errors with $K$ lags) only for the intercept and the coefficient on $N R C_{t}^{C}$ ( $L_{0}$ and $\left.L_{N R C}\right)$.

\begin{tabular}{clcccccc}
\hline \multirow{2}{*}{$R_{t+1: t+K}$} & & $I P_{t}^{H L}$ & $I P_{t}^{M C}$ & $I P_{t}^{C S}$ & $I P_{t}^{H L}$ & $I P_{t}^{M C}$ & $I P_{t}^{C S}$ \\
\hline \multirow{6}{*}{$K=12$} & $L_{0}$ & -4.37 & -4.25 & -3.94 & -4.37 & -4.25 & -3.94 \\
& $t$ & $(-1.76)$ & $(-2.66)$ & $(-2.35)$ & $(-1.67)$ & $(-2.63)$ & $(-2.31)$ \\
& $L_{N R C}$ & 7.19 & 4.50 & 4.26 & 5.29 & 3.46 & 4.39 \\
& $t$ & $(2.13)$ & $(2.30)$ & $(1.81)$ & $(1.81)$ & $(2.00)$ & $(2.35)$ \\
& $\zeta_{D Y}$ & 2.34 & 1.36 & -1.63 & & & \\
& $\zeta_{D S}$ & 2.53 & 0.42 & 2.05 & & & \\
& $\zeta_{T S}$ & 3.32 & 1.05 & 0.84 & & & \\
& $\zeta_{C A Y}$ & & & & -0.24 & -0.71 & -0.30 \\
& $R^{2}$ & 11.93 & 8.59 & 11.82 & 6.50 & 7.71 & 9.55 \\
\hline
\end{tabular}


Table OA.13: Alternative Measures of Inflation Risk

This table asks whether our results extend for alternative measures of inflation risk. In column one, we repeat our benchmark specification using ARMA(1,1)-innovations in inflation. In column two, we use raw inflation. In column three, we use an AR(1)model to proxy for inflation-innovations. In column four, we use the monthly change in annual inflation. In column five, we perform a truly out-of-sample exercise using real-time vintage CPI inflation. For this exercise, we skip a month after portfolio formation, thus taking into account the reporting delay in inflation data. In all cases, we calculate the returns of the High-minusLow inflation beta portfolio $\left(I P_{t}^{H L}\right)$. We present coefficient estimates from a regression of returns on the lagged nominal-real covariance $\left(N R C_{t}^{C}\right)$ at the twelve-month horizon, with corresponding $t$-statistics in parentheses based on Newey-West standard errors with $K=12$ lags.

\begin{tabular}{|c|c|c|c|c|c|c|}
\hline & & $\operatorname{ARMA}(1,1)$ & Inflation & $\operatorname{AR}(1)$ & Change in Annual Inflation & Real time \\
\hline$K=12$ & $\begin{array}{l}L_{0} \\
t \\
L_{N R C} \\
t \\
R^{2}\end{array}$ & $\begin{array}{c}-4.37 \\
(-1.68) \\
5.38 \\
(2.22) \\
6.65\end{array}$ & $\begin{array}{c}-3.89 \\
(-1.74) \\
7.35 \\
(3.25) \\
14.30\end{array}$ & $\begin{array}{c}-3.92 \\
(-1.73) \\
5.08 \\
(2.33) \\
7.26\end{array}$ & $\begin{array}{c}-4.88 \\
(-1.66) \\
6.87 \\
(2.56) \\
7.84\end{array}$ & $\begin{array}{c}-2.06 \\
(-0.96) \\
7.84 \\
(3.35) \\
16.12\end{array}$ \\
\hline
\end{tabular}

Table OA.14: Alternative Sorting Procedures

Each column in this table presents results from an alternative sorting procedure. We construct a High-minus-Low inflation beta decile portfolio by sorting stocks on inflation betas estimated using (i) Weighted Least Squares plus Shrinkage (WLS) or Ordinary Least Squares (OLS), (ii) a sort where each portfolio comes from a double sort on beta and size (Size) or a single sort on inflation beta (No Size), and (iii) $\operatorname{ARMA}(1,1)$ innovations in inflation (ARMA) or inflation itself (Inflation). The table presents the predictive regression of the inflation risk premium on the nominal-real covariance, as in Table 5 of the paper. The first column reports the original results as a benchmark.

\begin{tabular}{lcccccccc}
\hline & WLS & WLS & WLS & WLS & OLS & OLS & OLS & OLS \\
& $\begin{array}{c}\text { Size } \\
\text { ARMA }\end{array}$ & $\begin{array}{c}\text { Size } \\
\text { Inflation }\end{array}$ & $\begin{array}{c}\text { No Size } \\
\text { ARMA }\end{array}$ & $\begin{array}{c}\text { No Size } \\
\text { Inflation }\end{array}$ & $\begin{array}{c}\text { Size } \\
\text { ARMA }\end{array}$ & $\begin{array}{c}\text { Size } \\
\text { Inflation }\end{array}$ & $\begin{array}{c}\text { No Size } \\
\text { ARMA }\end{array}$ & $\begin{array}{c}\text { No Size } \\
\text { Inflation }\end{array}$ \\
& & & & & & & & \\
$L_{0}$ & -4.37 & -3.89 & -4.32 & -3.07 & -3.67 & -2.71 & -3.11 & -1.60 \\
$t$ & $(-1.68)$ & $(-1.74)$ & $(-1.50)$ & $(-1.27)$ & $(-1.46)$ & $(-1.18)$ & $(-1.10)$ & $(-0.60)$ \\
$L_{N R C}$ & 5.38 & 7.35 & 4.80 & 6.08 & 5.71 & 8.52 & 5.38 & 8.96 \\
$t$ & $(2.22)$ & $(3.25)$ & $(1.78)$ & $(2.59)$ & $(2.39)$ & $(3.80)$ & $(2.11)$ & $(3.69)$ \\
$R^{2}$ & 6.65 & 14.30 & 4.21 & 8.61 & 7.78 & 17.33 & 5.29 & 14.60 \\
\hline
\end{tabular}


Table OA.15: Predicting the Inflation Risk Premium with the Nominal-Real Covariance (Quarterly data)

This table is similar to Table 5 of the paper, except that we use quarterly instead of monthly data and, for brevity, we focus on the High-minus-Low inflation risk premium. We construct a High-minus-Low inflation beta portfolio by sorting stocks on the exposure in quarterly returns to quarterly $\operatorname{ARMA}(1,1)$-innovations in inflation. Moreover, we use quarterly inflation and quarterly consumption growth to estimate the nominal-real covariance. We then run the regression of Eq. (7). The nominal-real covariance is standardized and standard errors are Newey-West with $K$ lags.

\begin{tabular}{cccc}
\hline & & & \\
$R_{t+1: t+K}$ & $L_{0}$ & $L_{N R C}$ & $R^{2}$ \\
& & & \\
\hline$K=1 Q$ & -1.99 & 6.31 & 2.00 \\
& $(-0.66)$ & $(2.07)$ & \\
$K=2 Q$ & -1.92 & 6.25 & 3.90 \\
& $(-0.67)$ & $(2.26)$ & \\
$K=4 Q$ & -1.79 & 6.67 & 8.62 \\
& $(-0.69)$ & $(2.80)$ & \\
\hline
\end{tabular}

Table OA.16: Pooled Regressions: Inflation Betas Linear in the Nominal-Real Covariance

Similar to Panel A of Table 6, this table presents pooled regressions of returns on the inflation beta-sorted decile portfolios on inflation beta, the nominal-real covariance, and their interaction. In this case, inflation betas are estimated as a linear function of the nominal-real covariance, that is, $\beta_{\Pi, p, t}=\beta_{\Pi, p, t}^{0}+\beta_{\Pi, p, t}^{1} N R C_{t-1}^{C}$ (see Section 8. for more detail). We present estimated coefficients (with asymptotic Driscoll and Kraay (1998) standard errors in parentheses) from various pooled predictive regressions. To accommodate interpretation, $N R C_{t}^{C}$ is standardized and $\beta_{\Pi, p, t}$ is demeaned in the pool. We focus on the twelve-month horizon.

\begin{tabular}{lccccc}
\hline Model & {$[1]$} & {$[2]$} & {$[3]$} & {$[4]$} & {$[5]$} \\
& & & & & \\
\hline$L_{0}$ & 8.48 & 5.17 & 8.48 & 5.09 & 5.12 \\
$t$ & $(3.47)$ & $(2.24)$ & $(3.47)$ & $(2.18)$ & $(2.19)$ \\
$L_{\beta_{\Pi}}$ & 0.45 & -0.71 & 0.49 & -0.75 & -0.71 \\
$t$ & $(0.65)$ & $(-1.30)$ & $(0.71)$ & $(-1.34)$ & $(-1.25)$ \\
$L_{N R C}$ & -2.27 & -1.69 & -2.35 & -1.66 & -1.73 \\
$t$ & $(-0.68)$ & $(-0.71)$ & $(-0.70)$ & $(-0.70)$ & $(-0.73)$ \\
$L_{\beta_{\Pi} \times N R C}$ & & 1.64 & & & \\
$t$ & & $(4.25)$ & & & \\
$L_{\beta_{\Pi, p} \times N R C}$ & & & 2.72 & & 2.28 \\
$t$ & & & $(2.84)$ & & $(2.45)$ \\
$L_{\beta_{\Pi, t} \times N R C}$ & & & & 1.68 & 1.67 \\
$t$ & & & & $(4.13)$ & $(4.09)$ \\
$R^{2}$ & 0.89 & 11.36 & 1.60 & 10.95 & 11.44 \\
\hline
\end{tabular}


Table OA.17: Pooled Regressions Controlling for Monetary Policy Regimes

This table is similar to Panel A of Table 6 except that we now include a dummy for each monetary policy regime identified in Campbell et al. (2015): $M P R_{1}$ runs from the start of the sample to March 1977, $M P R_{2}$ runs from April 1977 to December 2000 , and $M P R_{3}$ runs from January 2001 to December 2014. We present estimated coefficients (with asymptotic Driscoll and Kraay (1998) standard errors in parentheses) from various pooled predictive regressions. In Model [1], we include only the dummies for the monetary policy regimes. In Model [2], we add inflation betas $\left(\beta_{\Pi, p, t}\right)$ and their interaction with the nominal-real covariance. In Model [3], we decompose the inflation beta for the interaction with the nominal-real covariance in a portfolioand time-specific component: $\widehat{\beta_{\Pi, p}}=T^{-1} \sum_{t=1}^{T} \beta_{\Pi, p, t}$ and $\widehat{\beta_{\Pi, t}}=10^{-1} \sum_{p=1}^{10} \beta_{\Pi, p, t}$. To accommodate interpretation, $N R C_{t}^{C}$ is standardized in the time series to have mean zero and standard deviation equal to one, whereas $\beta_{\Pi, p, t}$ is demeaned in the pool. We focus on the twelve-month horizon.

\begin{tabular}{lccc}
\hline Model & {$[1]$} & {$[2]$} & {$[3]$} \\
& & & \\
\hline$M P R_{1}$ & 1.30 & -1.17 & -1.30 \\
& $(0.20)$ & $(-0.18)$ & $(-0.20)$ \\
$M P R_{2}$ & 10.03 & 5.54 & 5.43 \\
& $(3.89)$ & $(1.86)$ & $(1.79)$ \\
$M P R_{3}$ & 11.03 & 10.16 & 10.13 \\
& $(2.18)$ & $(1.32)$ & $(1.32)$ \\
$L_{\beta_{\Pi}}$ & & -1.42 & -1.48 \\
& & $(-1.41)$ & $(-1.42)$ \\
$L_{N R C}$ & & -2.37 & -2.37 \\
& & $(-0.79)$ & $(-0.79)$ \\
$L_{\beta_{\Pi} \times N R C}$ & & 2.12 & \\
$L_{\beta_{\Pi, p} \times N R C}$ & & $(3.12)$ & \\
$L_{\beta_{\Pi, t} \times N R C}$ & & & 1.95 \\
$R^{2}$ & & & $(2.13)$ \\
& 2.72 & 10.19 & $(2.93)$ \\
& & & \\
\hline
\end{tabular}




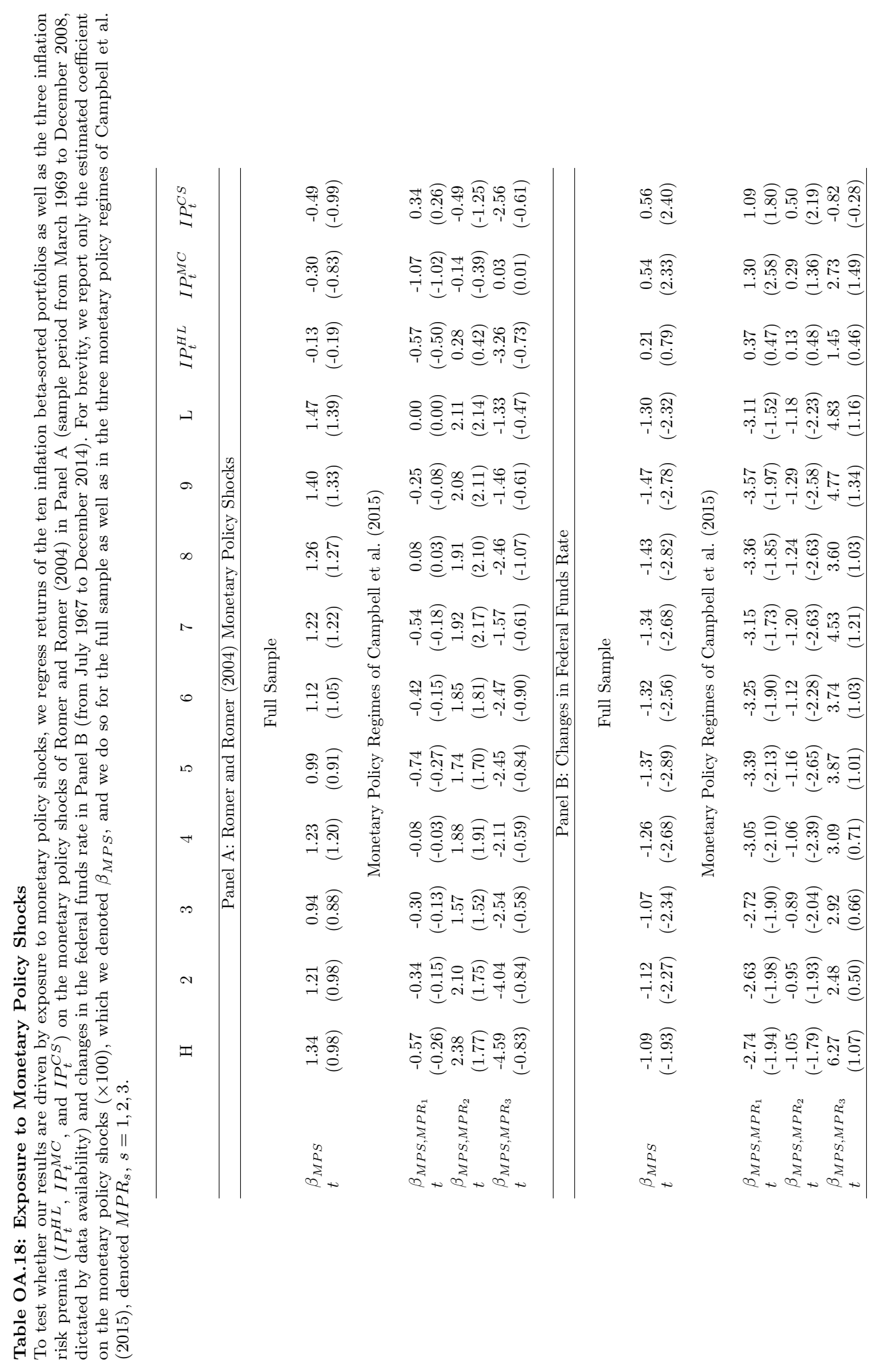


Table OA.19: Inflation Risk and Predictability in Excess Bond Return

Panel A shows summary statistics and the unconditional (full-sample) inflation exposure $\beta_{\Pi}$ for the excess returns of constant maturity Treasury bonds. Panel B shows time-series predictive regressions of these excess bond returns on the lagged nominalreal covariance in Panel B. The nominal-real covariance is lagged by 12 months and is standardized to have mean zero and variance one. We report for each regression the estimated intercepts $L_{0}$ and coefficients $L_{N R C}$ with corresponding $t$-statistics based on Newey-West standard errors with 12 lags in parenthesis. The sample period is July 1967 to December 2014.

\begin{tabular}{|c|c|c|c|c|c|c|c|}
\hline Maturity & 1 & 2 & 5 & 7 & 10 & 20 & 30 \\
\hline \multicolumn{8}{|c|}{ Panel A: Summary Statistics and Unconditional Inflation Exposure } \\
\hline $\begin{array}{l}\text { Avg. Ret. } \\
\text { St. Dev. } \\
\text { Sharpe } \\
\beta_{\Pi}\end{array}$ & $\begin{array}{c}1.10 \\
1.65 \\
0.67 \\
-0.25 \\
(-3.05)\end{array}$ & $\begin{array}{c}1.37 \\
2.91 \\
0.47 \\
-0.43 \\
(-3.02)\end{array}$ & $\begin{array}{c}2.19 \\
5.48 \\
0.40 \\
-0.90 \\
(-2.73)\end{array}$ & $\begin{array}{c}2.70 \\
6.68 \\
0.40 \\
-1.31 \\
(-2.97)\end{array}$ & $\begin{array}{c}2.54 \\
7.96 \\
0.32 \\
-1.41 \\
(-2.91)\end{array}$ & $\begin{array}{c}3.35 \\
10.70 \\
0.31 \\
-2.76 \\
(-3.67)\end{array}$ & $\begin{array}{c}3.14 \\
11.97 \\
0.26 \\
-3.27 \\
(-3.66)\end{array}$ \\
\hline \multicolumn{8}{|c|}{ Panel B: Predictability at Annual Horizon } \\
\hline$L_{0}$ & $\begin{array}{c}1.12 \\
(5.04)\end{array}$ & $\begin{array}{c}1.43 \\
(3.56)\end{array}$ & $\begin{array}{c}2.24 \\
(3.09)\end{array}$ & $\begin{array}{c}2.77 \\
(3.19)\end{array}$ & $\begin{array}{c}2.62 \\
(2.57)\end{array}$ & $\begin{array}{c}3.29 \\
(2.43)\end{array}$ & $\begin{array}{c}3.04 \\
(2.05)\end{array}$ \\
\hline$L_{N R C}$ & $\begin{array}{c}-0.65 \\
(-3.01)\end{array}$ & $\begin{array}{c}-0.71 \\
(-1.86)\end{array}$ & $\begin{array}{l}-0.70 \\
(-0.99)\end{array}$ & $\begin{array}{l}-0.61 \\
(-0.68)\end{array}$ & $\begin{array}{c}-0.72 \\
(-0.64)\end{array}$ & $\begin{array}{l}-0.81 \\
(-0.56)\end{array}$ & $\begin{array}{c}-0.41 \\
(-0.24)\end{array}$ \\
\hline$R^{2}$ & 11.63 & 4.60 & 1.29 & 0.57 & 0.56 & 0.35 & -0.07 \\
\hline
\end{tabular}

